KUNS-2458

YITP-13-72

\title{
Exact Results in Supersymmetric Field Theories on Manifolds with Boundaries
}

\author{
Sotaro Sugishita $*$ and Seiji Terashima \\ ${ }^{a}$ Department of Physics, Kyoto University, Kyoto 606-8502, Japan \\ ${ }^{b}$ Yukawa Institute for Theoretical Physics, Kyoto University, Kyoto 606-8502, Japan
}

\begin{abstract}
We construct supersymmetric gauge theories on some curved manifolds with boundaries. Our examples include a part of three-sphere and a part of two-sphere. We concentrate on Dirichlet boundary conditions. For these theories on the manifolds with the boundaries, we compute the partition functions and the Wilson loops exactly using the localization technique.
\end{abstract}

${ }^{*}$ sotaro(at)gauge.scphys.kyoto-u.ac.jp

${ }^{\dagger}$ terasima(at)yukawa.kyoto-u.ac.jp 


\section{Contents}

1 Introduction and Summary $\quad 2$

2 Three-dimensional theories 3

2.1 A 3D manifold with a boundary . . . . . . . . . . . . . . . . 3

2.2 3D supersymmetric field theories . . . . . . . . . . . . . . 4

2.2.1 The boundary condition .................... 6

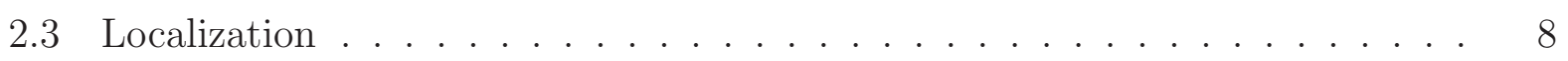

$2.3 .13 \mathrm{D}$ vector multiplet . . . . . . . . . . . . . 8

$2.3 .23 \mathrm{D}$ chiral multiplet ..................... 10

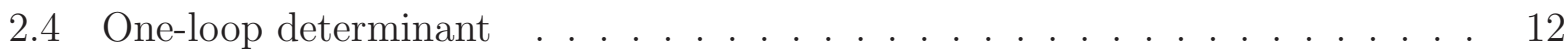

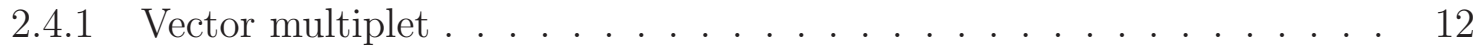

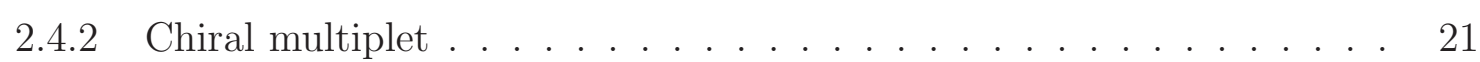

2.5 Partition functions and Wilson loops ................ 24

3 Two-dimensional theories $\quad 25$

3.1 A 2 D manifold with a boundary ......................... 25

3.2 2D supersymmetric field theories . . . . . . . . . . . . . 26

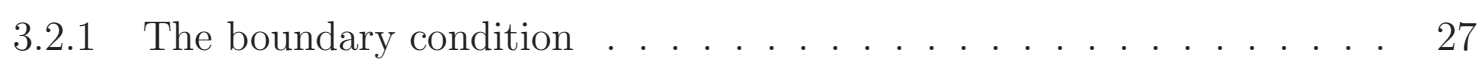

3.3 Localization . . . . . . . . . . . . . . . . . . . . . . . 28

3.3.1 2D vector multiplet . . . . . . . . . . . . . . . . 29

3.3 .2 2D chiral multiplet . . . . . . . . . . . . . . 30

3.4 One-loop determinant . . . . . . . . . . . . . . . . . . . . 31

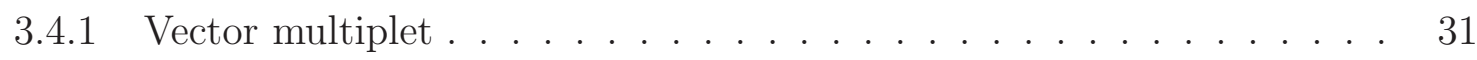

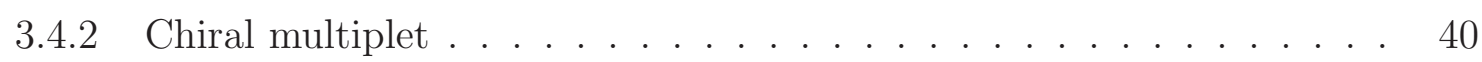

3.5 Partition functions and Wilson loops .................... 42

A Notations and useful formulas 


\section{Introduction and Summary}

In order to understand the dynamics of the gauge theory, we need to perform non-perturbative computations reliably. The analytical or exact results of them are especially important although they may be extraordinary difficult for generic gauge theories. The gauge theories with the supersymmetry (SUSY) are highly non-trivial examples of the theories in which exact results are obtained. The SUSY gauge theories themselves are important objects related to string theory, mathematics and many other areas. Even for the understanding the non-SUSY gauge theories, the SUSY gauge theories will be important because of the exact results and the physical consequences from them.

The exact results for the SUSY gauge theories have been mainly obtained using the holomorphy [1, 2, 3], which imposes strong constraints for the certain quantities. Recently, the more direct computations of the partition function and other operators using the so called localization technique has been extensively developed [4, 5]. In particular, in [5], the $4 \mathrm{D} \mathcal{N}=2$ SUSY gauge theories on a curved space were constructed and then the partition function and the Wilson loops are exactly computed using the localization technique. This method has advantages of the wide applicability and the straightforwardness. It has been applied for the SUSY gauge theories on various curved spaces with different dimensions [6]-39], however, the SUSY gauge theories on manifolds with boundaries have never been investigated using the localization technique. The exact results in such theories will be also highly important, for example, if we remember the recent application of the SUSY theories on $S^{2}$ as string world sheet actions [40].

In this paper, we explicitly construct the supersymmetric gauge theories on some curved manifolds with boundaries 3 Our examples include a part of $S^{3}$ with a torus boundary and a part of $S^{2}$ with a $S^{1}$ boundary. We use off-shell formulation of the SUSY and concentrate on Dirichlet boundary conditions. Actually, there seems to be no consistent Neumann-like

\footnotetext{
${ }^{3}$ The SUSY theories with boundaries on flat spaces have been considered in, for examples, [41, 42, 43] for $2 \mathrm{D}, 45,46,47$, 44] for 3D and [48] for 4D.
} 
boundary conditions with the SUSY if we impose the boundary condition which eliminates only the half of the fermions 4 For the SUSY theories, we compute the partition functions and the Wilson loops exactly using the localization technique.

Needless to say, our work is just a first step and there are many points which need further investigations: for examples, the interpretations and the applications of our results, the other boundary conditions, and extensions to other topologies and other dimensions. One important direction is to extend the ABJM model with the boundary condition representing the M5-branes [49, 50] to the curved manifold and compute some exact quantities because these might give us a clue for the M5-branes. We hope to report for the investigations of these topics in the future.

The organization of this paper is as follows: In section 2, we construct 3D SUSY field theories on the manifold with the torus boundary, which is constructed by cutting an $S^{3}$. The consistent boundary conditions we impose are the Dirichlet boundary conditions. Using the localization technique, we compute the partition function and the Wilson loop of the theories exactly. In section 3, we construct 2D SUSY field theories on the manifold with the circle boundary, which is constructed by cutting an $S^{2}$. The partition function and the Wilson loop for the theories are computed exactly.

The notations and some useful formulas are summarized in the Appendix A.

Note added: The authors thank K. Hori and M. Romo and D. Honda and T. Okuda for notifying them of submissions of related papers [51, 52].

\section{Three-dimensional theories}

\subsection{A 3D manifold with a boundary}

We will describe a three dimensional manifold with a boundary on which the supersymmetric field theories constructed. First we recall the round $S^{3}$, i.e. $\left(X_{0}{ }^{2}+X_{1}{ }^{2}+X_{2}{ }^{2}+X_{3}{ }^{2}=\ell^{2}\right.$ in $\mathbb{R}^{4}$ ). The coordinates we will use are

$$
\begin{aligned}
& X_{0}=\ell \cos \theta \cos \varphi, X_{1}=\ell \cos \theta \sin \varphi, \\
& X_{2}=\ell \sin \theta \cos \chi, X_{3}=\ell \sin \theta \sin \chi .
\end{aligned}
$$

\footnotetext{
${ }^{4}$ This assumption may be too strong. Indeed, it may be possible to use the boundary condition used in [42] even in the off-shell formulation or to introduce some boundary degrees of freedom, however, we leave such possibilities for future works.
} 
where $0 \leq \theta \leq \pi / 2,0 \leq \varphi \leq 2 \pi, 0 \leq \chi \leq 2 \pi$. The metric is given by

$$
\begin{aligned}
\mathrm{d} s^{2} & =\ell^{2}\left(\mathrm{~d} \theta^{2}+\cos ^{2} \theta \mathrm{d} \varphi^{2}+\sin ^{2} \theta \mathrm{d} \chi^{2}\right), \\
\sqrt{g} & =\ell^{3} \cos \theta \sin \theta
\end{aligned}
$$

We take the following dreibein:

$$
e^{1}=\ell \cos \theta \mathrm{d} \varphi, \quad e^{2}=\ell \sin \theta \mathrm{d} \chi, \quad e^{3}=\ell \mathrm{d} \theta
$$

and the gamma matrices:

$$
\gamma^{\varphi}=\frac{1}{\ell \cos \theta} \gamma^{1}, \quad \gamma^{\chi}=\frac{1}{\ell \sin \theta} \gamma^{2}, \quad \gamma^{\theta}=\frac{1}{\ell} \gamma^{3}
$$

Then, the manifold with the boundary is defined by just restricting the coordinate $\theta$ as

$$
0 \leq \theta \leq \theta_{0}
$$

where $0<\theta_{0} \leq \pi / 2$. Thus the boundary defined by $\theta=\theta_{0}$ is a torus parameterized by $\phi$ and $\chi$, except for $\theta_{0}=\pi / 2$. We will see that on the manifold with the boundary we can construct field theories with two supersymmetries (which are the half or $1 / 4$ of the supersymmetries of the $\mathcal{N}=2$ supersymmetric filed theories on the round $S^{3}$ ).

\subsection{D supersymmetric field theories}

Now we will construct the supersymmetric field theories on the manifold with the boundary.

First, we will summarize the SUSY transformations and the SUSY invariant Lagrangian of the $\mathcal{N}=2$ supersymmetric field theories on the round $S^{3}$ [7, ㅇ, 9]. We will write the derivative terms in the Lagrangians such that they are SUSY invariant on the manifold with the boundary. The Killing spinors on $S^{3}$ ware given by 5

$$
D_{\mu} \epsilon=\frac{i}{2 \ell} \gamma_{\mu} \epsilon,
$$

which is solved as:

$$
\epsilon=\frac{C_{1}}{\sqrt{2}}\left(\begin{array}{c}
-e^{-\frac{i}{2}(\varphi-\chi-\theta)} \\
e^{-\frac{i}{2}(\varphi-\chi+\theta)}
\end{array}\right)+\frac{C_{2}}{\sqrt{2}}\left(\begin{array}{c}
e^{\frac{i}{2}(\varphi-\chi+\theta)} \\
e^{\frac{i}{2}(\varphi-\chi-\theta)}
\end{array}\right)
$$

\footnotetext{
${ }^{5}$ There are the positive and the negative Killing spinors: $D_{\mu} \epsilon= \pm \frac{i}{2 \ell} \gamma_{\mu} \epsilon$. Either ones lead same result, thus we take the positive ones.
} 
in our basis.

The SUSY transformations of the vector multiplets with the Grassmann odd Killing spinor parameters $\epsilon, \bar{\epsilon}$ are the followings:

$$
\begin{aligned}
\delta A_{\mu} & =-\frac{i}{2}\left(\bar{\epsilon} \gamma_{\mu} \lambda-\bar{\lambda} \gamma_{\mu} \epsilon\right), \quad \delta \sigma=\frac{1}{2}(\bar{\epsilon} \lambda-\bar{\lambda} \epsilon) \\
\delta \lambda & =\left(\frac{1}{2} \gamma^{\mu \nu} F_{\mu \nu}-D+i D_{\mu} \sigma \gamma^{\mu}\right) \epsilon+\frac{2 i}{3} \sigma \gamma^{\mu} D_{\mu} \epsilon \\
\delta \bar{\lambda} & =\left(\frac{1}{2} \gamma^{\mu \nu} F_{\mu \nu}+D-i D_{\mu} \sigma \gamma^{\mu}\right) \bar{\epsilon}-\frac{2 i}{3} \sigma \gamma^{\mu} D_{\mu} \bar{\epsilon} \\
\delta D & =-\frac{i}{2} \bar{\epsilon}\left(\gamma^{\mu} D_{\mu} \lambda-[\lambda, \sigma]\right)-\frac{i}{2}\left(D_{\mu} \bar{\lambda} \gamma^{\mu}-[\bar{\lambda}, \sigma]\right) \epsilon-\frac{i}{6}\left(D_{\mu} \bar{\epsilon} \gamma^{\mu} \lambda+\bar{\lambda} \gamma^{\mu} D_{\mu} \epsilon\right),
\end{aligned}
$$

where all the fields are in the adjoint representation of the gauge group $G$ although we have not written the indices for it explicitly. Note that $a$ and $\bar{a}$ are independent fields for the Grassmann odd fields although usually $\bar{a}$ means a (complex) conjugate.

For the chiral multiplet of R-charge $q$, the SUSY transformations are:

$$
\begin{aligned}
\delta \phi & =\bar{\epsilon} \psi, \quad \delta \psi=\left(i D_{\mu} \phi \gamma^{\mu}+i \sigma \phi\right) \epsilon+\frac{2 i q}{3} \phi \gamma^{\mu} D_{\mu} \epsilon+\bar{\epsilon} F, \\
\delta \bar{\phi} & =\epsilon \bar{\psi}, \quad \delta \bar{\psi}=\left(i D_{\mu} \bar{\phi} \gamma^{\mu}+i \bar{\phi} \sigma\right) \bar{\epsilon}+\frac{2 i q}{3} \bar{\phi} \gamma^{\mu} D_{\mu} \bar{\epsilon}+\epsilon \bar{F}, \\
\delta F & =\epsilon\left(i \gamma^{\mu} D_{\mu} \psi-i \sigma \psi-i \lambda \phi\right)+\frac{i}{3}(2 q-1) D_{\mu} \epsilon \gamma^{\mu} \psi, \\
\delta \bar{F} & =\bar{\epsilon}\left(i \gamma^{\mu} D_{\mu} \bar{\psi}-i \bar{\psi} \sigma+i \bar{\phi} \bar{\lambda}\right)+\frac{i}{3}(2 q-1) D_{\mu} \bar{\epsilon} \gamma^{\mu} \bar{\psi},
\end{aligned}
$$

where $\phi, \psi, F$ are in a representation $R$ of $G$ and $\bar{\phi}, \bar{\psi}, \bar{F}$ are in the complex conjugate representation of $R$.

There are several invariant actions under the SUSY transformations (2.9) $-(2.10)$. The one is the Yang-Mills Lagrangian:6

$$
\begin{aligned}
\frac{1}{g_{\mathrm{YM}}^{2}} \mathcal{L}_{\mathrm{YM}}=\frac{1}{g_{\mathrm{YM}}^{2}} \operatorname{Tr}( & \frac{1}{4} F_{\mu \nu} F^{\mu \nu}+\frac{1}{2} D_{\mu} \sigma D^{\mu} \sigma+\frac{1}{2}(D+\sigma / \ell)^{2} \\
& \left.+\frac{i}{4} \bar{\lambda} \gamma^{\mu} D_{\mu} \lambda+\frac{i}{4} \lambda \gamma^{\mu} D_{\mu} \bar{\lambda}+\frac{i}{2} \bar{\lambda}[\sigma, \lambda]-\frac{1}{4 \ell} \bar{\lambda} \lambda\right),
\end{aligned}
$$

where $g_{\mathrm{YM}}$ is the coupling constant. Another one is the Chern-Simons term:

$$
\mathcal{L}_{\mathrm{CS}}=i \frac{k}{4 \pi} \operatorname{Tr}\left(\varepsilon^{\mu \nu \lambda}\left(A_{\mu} \partial_{\nu} A_{\lambda}-\frac{2 i}{3} A_{\mu} A_{\nu} A_{\lambda}\right)-\bar{\lambda} \lambda+2 D \sigma\right)
$$

\footnotetext{
${ }^{6}$ Note that the fermion kinetic terms are symmetrical with respect to $\lambda$ and $\bar{\lambda}$. In the other Lagrangians, we also use the symmetric fermion kinetic terms.
} 
where the level $k$ is an integer for the theory on $S^{3}$. We can also construct the Fayet Iliopoulos (FI) term:

$$
\mathcal{L}_{\mathrm{FI}}=\frac{i \zeta}{\pi \ell} \operatorname{Tr}(D-\sigma / \ell)
$$

For the chiral multiplets, the matter kinetic terms are given by

$$
\begin{aligned}
\mathcal{L}_{\text {mat }}= & D_{\mu} \bar{\phi} D^{\mu} \phi+\bar{\phi} \sigma^{2} \phi+\frac{i(2 q-1)}{\ell} \bar{\phi} \sigma \phi+\frac{q(2-q)}{\ell^{2}} \bar{\phi} \phi+i \bar{\phi} D \phi+\bar{F} F \\
& -\frac{i}{2} \bar{\psi} \gamma^{\mu} D_{\mu} \psi+\frac{i}{2} D_{\mu} \bar{\psi} \gamma^{\mu} \psi+i \bar{\psi} \sigma \psi-\frac{(2 q-1)}{2 \ell} \bar{\psi} \psi+i \bar{\psi} \lambda \phi-i \bar{\phi} \bar{\lambda} \psi .
\end{aligned}
$$

We can also have the superpotential terms (without derivatives) which are same form as the ones in the flat space. Note that the bosonic kinetic terms for the chiral multiplet are written as

$$
\begin{aligned}
& D_{\mu} \bar{\phi} D^{\mu} \phi+\bar{\phi} \sigma^{2} \phi+\frac{i(2 q-1)}{\ell} \bar{\phi} \sigma \phi+\frac{q(2-q)}{\ell^{2}} \bar{\phi} \phi+i \bar{\phi} D \phi+\bar{F} F+\frac{1}{2 g_{Y M}^{2}}(D+\sigma / \ell)^{2} \\
& =\left|D_{\mu} \phi\right|^{2}+\bar{\phi}\left(\sigma+\frac{i}{\ell}(q-1)\right)^{2} \phi+\frac{1}{\ell^{2}}|\phi|^{2}+\frac{1}{2}\left(\frac{1}{g_{Y M}}(D+\sigma / \ell)+i g_{Y M} \phi \bar{\phi}\right)^{2}+\frac{g_{Y M}^{2}}{2}|\phi|^{4}+|F|^{2}
\end{aligned}
$$

which can be positive definite if we shift $\sigma, D$ appropriately.

\subsubsection{The boundary condition}

If one imposes the boundary conditions on fields, the supersymmetry which preserves these boundary conditions is remain. There are possible candidates for consistent supersymmetric boundary conditions for our theories. It is an important question which ones indeed works. In this paper, however, we study the Dirichlet condition only as a first step.

Denoting $\tilde{\mu}$ as the coordinates tangent to the boundary, i.e. $\{\varphi, \chi\}$, our ansatz for the boundary conditions for the vector multiplet is:

$$
\begin{gathered}
\left.A_{\tilde{\mu}}\right|_{\theta=\theta_{0}}=a_{\tilde{\mu}}, \\
\left.\sigma\right|_{\theta=\theta_{0}}=\sigma_{0}, \\
\left.\ell e^{i(\varphi-\chi)} \gamma^{\theta} \lambda\right|_{\theta=\theta_{0}}=\left.\bar{\lambda}\right|_{\theta=\theta_{0}},
\end{gathered}
$$

where $a_{\tilde{\mu}}$ and $\sigma_{0}$ are constants and in the Cartan part of the adjoint representation. We do not impose any conditions for other fields ( $A_{\theta}$ and $D$ ). Note that the conditions for the bosons may be covariantly represented as $\left.F_{\tilde{\mu} \tilde{\nu}}\right|_{\theta=\theta_{0}}=0$ and $\left.D_{\tilde{\mu}} \sigma\right|_{\theta=\theta_{0}}=0$. We can see 
that these boundary conditions indeed preserve the half of the supersymmetries which are generated by the Killing spinors satisfying the relation

$$
-\ell e^{i(\varphi-\chi)} \gamma^{\theta} \epsilon=\bar{\epsilon}
$$

In appendix $\mathrm{B}$, we show that the actions for the vector multiplet are invariant under these supersymmetries with the above boundary conditions.

For chiral multiplets, we take the following boundary condition:

$$
\begin{aligned}
& \left.\phi\right|_{\theta=\theta_{0}}=0,\left.\quad e^{\frac{i \theta_{0}}{2} \gamma^{3}} \gamma^{1} e^{-\frac{i \theta_{0}}{2} \gamma^{3}} \psi\right|_{\theta=\theta_{0}}=\left.\psi\right|_{\theta=\theta_{0}}, \\
& \left.\bar{\phi}\right|_{\theta=\theta_{0}}=0,\left.\quad e^{\frac{i \theta_{0}}{2} \gamma^{3}} \gamma^{1} e^{-\frac{i \theta_{0}}{2} \gamma^{3}} \bar{\psi}\right|_{\theta=\theta_{0}}=-\left.\bar{\psi}\right|_{\theta=\theta_{0}} .
\end{aligned}
$$

Then these boundary conditions for the chiral multiplet preserve another half of the supersymmetries which is generated by the Killing spinors satisfying the relations

$$
e^{\frac{i \theta}{2} \gamma^{3}} \gamma^{1} e^{-\frac{i \theta}{2} \gamma^{3}} \epsilon=-\epsilon, \quad e^{\frac{i \theta}{2} \gamma^{3}} \gamma^{1} e^{-\frac{i \theta}{2} \gamma^{3}} \bar{\epsilon}=\bar{\epsilon}
$$

We also show that the matter kinetic action is invariant under the SUSY with the boundary condition in Appendix B.

We will consider SUSY theories with both vector and chiral multiplets, thus only the 1/4 SUSY will remain. The Grassmann even Killing spinors satisfying the both relations are given by

$$
\epsilon=\frac{1}{\sqrt{2}}\left(\begin{array}{c}
-e^{-\frac{i}{2}(\varphi-\chi-\theta)} \\
e^{-\frac{i}{2}(\varphi-\chi+\theta)}
\end{array}\right) \quad \text { and } \quad \bar{\epsilon}=\frac{1}{\sqrt{2}}\left(\begin{array}{c}
e^{\frac{i}{2}(\varphi-\chi+\theta)} \\
e^{\frac{i}{2}(\varphi-\chi-\theta)}
\end{array}\right)
$$

We can compute the bi-linears of the Grassmann even spinors:

$$
\begin{aligned}
\bar{\epsilon} \epsilon & =1, \\
\bar{\epsilon} \gamma^{a} \epsilon & =(-\cos \theta, \sin \theta, 0) \equiv v^{a}, \\
\epsilon \gamma^{a} \epsilon & =(i \sin \theta, i \cos \theta, 1) e^{-i(\varphi-\chi)} \equiv v_{+}^{a}, \\
\bar{\epsilon} \gamma^{a} \bar{\epsilon} & =(i \sin \theta, i \cos \theta,-1) e^{+i(\varphi-\chi)} \equiv v_{-}^{a},
\end{aligned}
$$

which will be used later.

We should check also that the boundary conditions are consistent with the variational principle. The surface terms from variation of the Yang-Mills action are

$$
\frac{1}{g_{\mathrm{YM}}^{2}} \int_{\theta=\theta_{0}} \mathrm{~d} \varphi \mathrm{d} \chi\left[\sqrt{g} \operatorname{Tr}\left(\delta A_{\nu} F^{\theta \nu}+\delta \sigma D^{\theta} \sigma+\frac{i}{4} \bar{\lambda} \gamma^{\theta} \delta \lambda+\frac{i}{4} \lambda \gamma^{\theta} \delta \bar{\lambda}\right)\right],
$$


which indeed vanish for the boundary conditions. Note that we do not need to introduce boundary terms because of our choice of the kinetic terms of the Lagrangian. We can see that the surface terms from variation of the Chern-Simons action,

$$
i \int_{\theta=\theta_{0}} \mathrm{~d} \varphi \mathrm{d} \chi\left[\frac{k}{4 \pi} \operatorname{Tr}\left(A_{\chi} \delta A_{\varphi}-A_{\varphi} \delta A_{\chi}\right)\right]
$$

and the matter kinetic terms,

$$
\int_{\theta=\theta_{0}} \mathrm{~d} \varphi \mathrm{d} \chi\left[\sqrt{g}\left(\delta \bar{\phi} D^{\theta} \phi+D^{\theta} \bar{\phi} \delta \phi-\frac{i}{2} \bar{\psi} \gamma^{\theta} \delta \psi+\frac{i}{2} \delta \bar{\psi} \gamma^{\theta} \psi\right)\right],
$$

vanish for the boundary conditions.

\subsection{Localization}

In order to compute the partition function exactly using the localization technique, we will introduce the $\delta$-exact Lagrangian $\delta V$ as in [5]. Note that we need to keep the total divergence terms which have been neglected for the theories on the manifold without boundaries. The localization technique implies that the expectation values of the $\delta$-closed operators are not changed by deforming the action $S \rightarrow S+t \int \delta V$, and in the $t \rightarrow \infty$ limit, the saddle point approximation becomes exact.

\subsection{1. $3 \mathrm{D}$ vector multiplet}

For the vector multiplet, we take the following $\delta$-exact term (ignoring the trace of the gauge indices for notational convenience):

$$
\delta V_{\text {vector }}=\frac{1}{4} \delta\left(\left(\delta^{\prime} \lambda\right)^{\dagger} \lambda+\bar{\lambda}\left(\delta^{\prime} \bar{\lambda}\right)^{\dagger}\right)
$$

where $\delta, \delta^{\prime}$ are the same SUSY transformations with the Grassmann odd Killing spinors $\epsilon, \bar{\epsilon}$. More precisely, we will add $t \int \delta V_{\text {vector }}$ with the Grassmann even Killing spinors to the action. For the computational convenience, we use the Grassmann odd spinors. After the computations, we will replace, for examples, $\bar{\epsilon}^{\prime} \epsilon=-\bar{\epsilon} \epsilon^{\prime} \rightarrow 1$ and $\bar{\epsilon}^{\prime} \gamma^{\mu} \epsilon=-\bar{\epsilon} \gamma^{\mu} \epsilon^{\prime} \rightarrow v^{\mu}$. We have also defined

$$
\begin{aligned}
& (\delta \lambda)^{\dagger}=\bar{\epsilon}\left(-\frac{1}{2} \gamma^{\mu \nu} F_{\mu \nu}-D-i \gamma^{\mu} D_{\mu} \sigma-\frac{\sigma}{\ell}\right) \\
& (\delta \bar{\lambda})^{\dagger}=\left(-\frac{1}{2} \gamma^{\mu \nu} F_{\mu \nu}-D+i D_{\mu} \sigma \gamma^{\mu}-\frac{\sigma}{\ell}\right) \epsilon .
\end{aligned}
$$


With these definitions, we find that $(\delta \lambda)^{\dagger} \delta \lambda=\sum_{\alpha}\left|(\delta \lambda)_{\alpha}\right|^{2}$, which is manifestly positive definite. For the bosonic part of the $\delta$-exact term, we can show that

$$
\begin{aligned}
& \left(\delta^{\prime} \lambda\right)^{\dagger} \delta \lambda=\bar{\epsilon}^{\prime} \epsilon\left(\frac{1}{2} F_{\mu \nu} F^{\mu \nu}+D_{\mu} \sigma D^{\mu} \sigma+(D+\sigma / \ell)^{2}+\varepsilon^{\mu \nu \rho} F_{\mu \nu} D_{\rho} \sigma\right) \\
& \delta \bar{\lambda}\left(\delta^{\prime} \bar{\lambda}\right)^{\dagger}=\bar{\epsilon}^{\prime} \epsilon\left(\frac{1}{2} F_{\mu \nu} F^{\mu \nu}+D_{\mu} \sigma D^{\mu} \sigma+(D+\sigma / \ell)^{2}-\varepsilon^{\mu \nu \rho} F_{\mu \nu} D_{\rho} \sigma\right)
\end{aligned}
$$

where we have used $\bar{\epsilon} \epsilon^{\prime}=-\bar{\epsilon}^{\prime} \epsilon$. Therefore,

$$
\begin{aligned}
\left(\delta^{\prime} \lambda\right)^{\dagger} \delta \lambda+\delta \bar{\lambda}\left(\delta^{\prime} \bar{\lambda}\right)^{\dagger} & =4 \bar{\epsilon}^{\prime} \epsilon\left(\frac{1}{4} F_{\mu \nu} F^{\mu \nu}+\frac{1}{2} D_{\mu} \sigma D^{\mu} \sigma+\frac{1}{2}(D+\sigma / \ell)^{2}\right) \\
& =4 \bar{\epsilon}^{\prime} \epsilon \mathcal{L}_{\mathrm{YM}}^{\text {boson }}
\end{aligned}
$$

For the fermionic part of the $\delta$-exact term, we can show that

$$
\delta\left(\left(\delta^{\prime} \lambda\right)^{\dagger}\right) \lambda=-\frac{1}{2} \delta F_{\mu \nu} \bar{\epsilon}^{\prime} \gamma^{\mu \nu} \lambda-i \delta\left(D_{\mu} \sigma\right) \bar{\epsilon}^{\prime} \gamma^{\mu} \lambda-(\delta D+\delta \sigma / \ell) \bar{\epsilon}^{\prime} \lambda
$$

where

$$
\begin{aligned}
\delta F_{\mu \nu} & =\delta\left(\partial_{\mu} A_{\nu}-\partial_{\nu} A_{\mu}-i\left[A_{\mu}, A_{\nu}\right]\right) \\
& =-\frac{i}{2}\left(\bar{\epsilon} \gamma_{\nu} D_{\mu} \lambda-\bar{\epsilon} \gamma_{\mu} D_{\nu} \lambda+\epsilon \gamma_{\nu} D_{\mu} \bar{\lambda}-\epsilon \gamma_{\mu} D_{\nu} \bar{\lambda}\right)-\frac{1}{2 \ell}\left(\bar{\epsilon} \gamma_{\mu \nu} \lambda+\epsilon \gamma_{\mu \nu} \bar{\lambda}\right), \\
\delta\left(D_{\mu} \sigma\right) & =\delta\left(\partial_{\mu} \sigma-i\left[A_{\mu}, \sigma\right]\right) \\
& =\frac{1}{2}\left(\bar{\epsilon} D_{\mu} \lambda-\epsilon D_{\mu} \bar{\lambda}-\bar{\epsilon} \gamma_{\mu}[\lambda, \sigma]-\epsilon \gamma_{\mu}[\bar{\lambda}, \sigma]\right)-\frac{i}{4 \ell}\left(\bar{\epsilon} \gamma_{\mu} \lambda-\epsilon \gamma_{\mu} \bar{\lambda}\right), \\
\delta D+\delta \sigma / \ell & =-\frac{i}{2} \bar{\epsilon}\left(\gamma^{\mu} D_{\mu} \lambda-[\lambda, \sigma]+i \lambda /(2 \ell)\right)-\frac{i}{2} \epsilon\left(-\gamma^{\mu} D_{\mu} \bar{\lambda}-[\bar{\lambda}, \sigma]-i \bar{\lambda} /(2 \ell)\right),
\end{aligned}
$$

which are summed up to

$$
\delta\left(\left(\delta^{\prime} \lambda\right)^{\dagger}\right) \lambda=\bar{\epsilon}^{\prime} \epsilon\left(i \lambda \gamma^{\mu} D_{\mu} \bar{\lambda}+i \bar{\lambda}[\sigma, \lambda]-\frac{1}{2 \ell} \bar{\lambda} \lambda\right)+\left(\bar{\epsilon}^{\prime} \gamma^{\mu} \bar{\epsilon}\right)\left(i\left(D_{\mu} \lambda\right) \lambda\right) .
$$

In (2.41), the last term is total derivative and it becomes a surface term:

$$
i\left(\bar{\epsilon}^{\prime} \gamma^{\theta} \bar{\epsilon}\right)(\lambda \lambda)\left|=i \bar{\epsilon}^{\prime} \epsilon\left(\bar{\lambda} \gamma^{\theta} \lambda\right)\right|
$$

where we have used (2.18) and (2.19). Similarly, we find that

$$
\begin{aligned}
\bar{\lambda} \delta\left(\left(\delta^{\prime} \bar{\lambda}\right)^{\dagger}\right) & =-\frac{1}{2} \bar{\lambda} \gamma^{\mu \nu} \bar{\epsilon}^{\prime} \delta F_{\mu \nu}+i \bar{\lambda} \gamma^{\mu} \epsilon^{\prime} \delta\left(D_{\mu} \sigma\right)-\bar{\lambda} \epsilon^{\prime}(\delta D+\delta \sigma / \ell) \\
& =\bar{\epsilon}^{\prime} \epsilon\left(i \bar{\lambda} \gamma^{\mu} D_{\mu} \lambda+i \bar{\lambda}[\sigma, \lambda]-\frac{1}{2 \ell} \bar{\lambda} \lambda\right)-\left(\epsilon^{\prime} \gamma^{\mu} \epsilon\right)\left(i\left(D_{\mu} \bar{\lambda}\right) \bar{\lambda}\right)
\end{aligned}
$$

where the last term is also a total derivative and it becomes a surface term:

$$
i\left(\epsilon^{\prime} \gamma^{\theta} \epsilon\right)(\bar{\lambda} \bar{\lambda})\left|=i \bar{\epsilon}^{\prime} \epsilon\left(\bar{\lambda} \gamma^{\theta} \lambda\right)\right|
$$


Thus, the fermionic part of $\delta$-exact term is

$$
\begin{aligned}
\delta\left(\left(\delta^{\prime} \lambda\right)^{\dagger}\right) \lambda+\bar{\lambda} \delta\left(\left(\delta^{\prime} \bar{\lambda}\right)^{\dagger}\right) & =\bar{\epsilon}^{\prime} \epsilon\left(i \bar{\lambda} \gamma^{\mu} D_{\mu} \lambda+i \lambda \gamma^{\mu} D_{\mu} \bar{\lambda}+2 i \bar{\lambda}[\sigma, \lambda]-\frac{1}{\ell} \bar{\lambda} \lambda\right) \\
& =4 \bar{\epsilon}^{\prime} \epsilon \mathcal{L}_{\mathrm{YM}}^{\text {fermion }},
\end{aligned}
$$

and there is no surface term.

Therefore, the $\delta$-exact Lagrangian $\delta V_{\text {vector }}=\mathcal{L}_{\mathrm{YM}}^{\text {boson }}+\mathcal{L}_{\mathrm{YM}}^{\text {fermion }}$, is same as the Yang-Mills Lagrangian and there are no surface terms. The saddle points of the bosonic part of $\delta V_{\text {vector }}$ are given by

$$
F_{\mu \nu}=0, \quad D_{\mu} \sigma=0, \quad D=-\frac{\sigma}{l},
$$

which implies that $A_{\theta}=0, A_{\tilde{\mu}}=a_{\tilde{\mu}}, \sigma=\sigma_{0}$ where $a_{\tilde{\mu}}$ and $\sigma_{0}$ are constants satisfying $\left[\sigma_{0}, a_{\tilde{\mu}}\right]=0$ in an appropriate gauge.

\subsubsection{D chiral multiplet}

For the chiral multiplet, we consider the following $\delta$-exact term:

$$
\delta V_{\text {chiral }}=\frac{1}{2} \delta\left[\left(\delta^{\prime} \psi\right)^{\dagger} \psi+\bar{\psi}\left(\delta^{\prime} \bar{\psi}\right)^{\dagger}\right]+\frac{q-1}{\ell} \delta\left[\bar{\phi} \delta^{\prime} \phi-\left(\delta^{\prime} \bar{\phi}\right) \phi\right],
$$

where we have defined

$$
\begin{aligned}
& \left(\delta^{\prime} \psi\right)^{\dagger} \equiv \bar{\epsilon}^{\prime}\left(-i D_{\mu} \bar{\phi} \gamma^{\mu}-i \bar{\phi} \sigma-\frac{q}{\ell} \bar{\phi}\right)-\epsilon^{\prime} \bar{F} \\
& \left(\delta^{\prime} \bar{\psi}\right)^{\dagger} \equiv\left(-i D_{\mu} \phi \gamma^{\mu}+i \sigma \phi+\frac{q}{\ell} \phi\right) \epsilon^{\prime}+\bar{\epsilon}^{\prime} F
\end{aligned}
$$

The second term on the right hand side of (2.48) is added to simplify the calculation of the one-loop determinant. We will compute the bosonic part of the $\delta$-exact term first. We can see that

$$
\begin{aligned}
\left(\delta^{\prime} \psi\right)^{\dagger} \delta \psi= & \bar{\epsilon}^{\prime} \epsilon\left(D_{\mu} \bar{\phi} D^{\mu} \phi+\bar{\phi} \sigma^{2} \phi+\frac{q^{2}}{\ell^{2}} \bar{\phi} \phi+\bar{F} F\right) \\
& +\bar{\epsilon}^{\prime} \gamma^{\mu} \epsilon\left(i \varepsilon_{\mu \nu \rho} D^{\nu} \bar{\phi} D^{\rho} \phi+D_{\mu} \bar{\phi} \sigma \phi+i \frac{q}{\ell} D_{\mu} \bar{\phi} \phi+\bar{\phi} \sigma D_{\mu} \phi-i \frac{q}{\ell} \bar{\phi} D_{\mu} \phi\right) \\
& -i \epsilon^{\prime} \gamma^{\mu} \epsilon \bar{F} D_{\mu} \phi-i \bar{\epsilon}^{\prime} \gamma^{\mu} \bar{\epsilon} D_{\mu} \bar{\phi} F, \\
\delta \bar{\psi}\left(\delta^{\prime} \bar{\psi}\right)^{\dagger}= & \bar{\epsilon}^{\prime} \epsilon\left(D_{\mu} \bar{\phi} D^{\mu} \phi+\bar{\phi} \sigma^{2} \phi+\frac{q^{2}}{\ell^{2}} \bar{\phi} \phi+\bar{F} F\right) \\
& +\bar{\epsilon}^{\prime} \gamma^{\mu} \epsilon\left(i \varepsilon_{\mu \nu \rho} D^{\nu} \bar{\phi} D^{\rho} \phi-D_{\mu} \bar{\phi} \sigma \phi+i \frac{q}{\ell} D_{\mu} \bar{\phi} \phi-\bar{\phi} \sigma D_{\mu} \phi-i \frac{q}{\ell} \bar{\phi} D_{\mu} \phi\right) \\
& +i \epsilon^{\prime} \gamma^{\mu} \epsilon \bar{F} D_{\mu} \phi+i \bar{\epsilon}^{\prime} \gamma^{\mu} \bar{\epsilon} D_{\mu} \bar{\phi} F,
\end{aligned}
$$


which give

$$
\begin{aligned}
\left(\delta^{\prime} \psi\right)^{\dagger} \delta \psi+\delta \bar{\psi}\left(\delta^{\prime} \bar{\psi}\right)^{\dagger}= & 2 \bar{\epsilon}^{\prime} \epsilon\left(D_{\mu} \bar{\phi} D^{\mu} \phi+\bar{\phi} \sigma^{2} \phi+\frac{q^{2}}{\ell^{2}} \bar{\phi} \phi+\bar{F} F\right) \\
& +2 i \bar{\epsilon}^{\prime} \gamma^{\mu} \epsilon\left(\varepsilon_{\mu \nu \rho} D^{\nu} \bar{\phi} D^{\rho} \phi+\frac{q}{\ell} D_{\mu} \bar{\phi} \phi-\frac{q}{\ell} \bar{\phi} D_{\mu} \phi\right)
\end{aligned}
$$

We can also obtain

$$
\bar{\phi}\left(\delta \delta^{\prime} \phi\right)-\left(\delta \delta^{\prime} \bar{\phi}\right) \phi=2 \bar{\epsilon}^{\prime} \epsilon\left(i \bar{\phi} \sigma \phi-\frac{q}{\ell} \bar{\phi} \phi\right)+i \bar{\epsilon}^{\prime} \gamma^{\mu} \epsilon\left(\bar{\phi} D_{\mu} \phi-D_{\mu} \bar{\phi} \phi\right) .
$$

For the fermionic part of $\delta$-exact term, we can see that

$$
\begin{aligned}
\delta\left(\left(\delta^{\prime} \psi\right)^{\dagger}\right) \psi= & \bar{\epsilon}^{\prime} \epsilon\left(i D_{\mu} \bar{\psi} \gamma^{\mu} \psi+i \bar{\psi} \sigma \psi-\frac{3 i}{2} \bar{\phi} \bar{\lambda} \psi-\frac{1}{2 \ell} \bar{\psi} \psi\right) \\
& +\frac{i}{2} \bar{\epsilon}^{\prime} \gamma^{\mu} \epsilon\left(2 i \varepsilon_{\mu \nu \rho} D^{\nu} \bar{\psi} \gamma^{\rho} \psi+\bar{\phi} \bar{\lambda} \gamma_{\mu} \psi-i \frac{2 q}{\ell} \bar{\psi} \gamma_{\mu} \psi\right) \\
& +\frac{i}{2} \bar{\epsilon}^{\prime} \gamma^{\mu} \bar{\epsilon} \bar{\phi} \lambda \gamma_{\mu} \psi, \\
\bar{\psi} \delta\left(\left(\delta^{\prime} \bar{\psi}\right)^{\dagger}\right)= & -\bar{\epsilon}^{\prime} \epsilon\left(i \bar{\psi} \gamma^{\mu} D_{\mu} \psi-i \bar{\psi} \sigma \psi-\frac{3 i}{2} \bar{\psi} \lambda \phi+\frac{1}{2 \ell} \bar{\psi} \psi\right) \\
& -\frac{i}{2} \bar{\epsilon}^{\prime} \gamma^{\mu} \epsilon\left(-2 i \varepsilon_{\mu \nu \rho} \bar{\psi} \gamma^{\rho} D^{\nu} \psi+\bar{\psi} \gamma_{\mu} \lambda \phi+i \frac{2 q}{\ell} \bar{\psi} \gamma_{\mu} \psi\right) \\
& -\frac{i}{2} \epsilon^{\prime} \gamma^{\mu} \epsilon \bar{\psi} \gamma_{\mu} \bar{\lambda} \phi,
\end{aligned}
$$

thus we find that

$$
\begin{aligned}
\delta( & \left.\left(\delta^{\prime} \psi\right)^{\dagger}\right) \psi+\bar{\psi} \delta\left(\left(\delta^{\prime} \bar{\psi}\right)^{\dagger}\right) \\
= & \bar{\epsilon}^{\prime} \epsilon\left(i D_{\mu} \bar{\psi} \gamma^{\mu} \psi-i \bar{\psi} \gamma^{\mu} D_{\mu} \psi+2 i \bar{\psi} \sigma \psi-\frac{3 i}{2} \bar{\phi} \bar{\lambda} \psi+\frac{3 i}{2} \bar{\psi} \lambda \phi-\frac{1}{\ell} \bar{\psi} \psi\right) \\
& +\frac{i}{2} \bar{\epsilon}^{\prime} \gamma^{\mu} \epsilon\left(2 i \varepsilon_{\mu \nu \rho} D^{\nu} \bar{\psi} \gamma^{\rho} \psi+2 i \varepsilon_{\mu \nu \rho} \bar{\psi} \gamma^{\rho} D^{\nu} \psi+\bar{\phi} \bar{\lambda} \gamma_{\mu} \psi-\bar{\psi} \gamma_{\mu} \lambda \phi-i \frac{4 q}{\ell} \bar{\psi} \gamma_{\mu} \psi\right) \\
& -\frac{i}{2} \epsilon^{\prime} \gamma^{\mu} \epsilon \bar{\psi} \gamma_{\mu} \bar{\lambda} \phi+\frac{i}{2} \bar{\epsilon}^{\prime} \gamma^{\mu} \bar{\epsilon} \bar{\phi} \lambda \gamma_{\mu} \psi .
\end{aligned}
$$

We also compute

$$
\delta \bar{\phi} \delta^{\prime} \phi-\delta^{\prime} \bar{\phi} \delta \phi=-\bar{\epsilon}^{\prime} \epsilon \bar{\psi} \psi-\bar{\epsilon}^{\prime} \gamma^{\mu} \epsilon \bar{\psi} \gamma_{\mu} \psi
$$

Therefore, the SUSY exact term we take is

$$
\begin{aligned}
\delta V_{\text {chiral }}= & \bar{\epsilon}^{\prime} \epsilon\left(D_{\mu} \bar{\phi} D^{\mu} \phi+\bar{\phi} \sigma^{2} \phi+2 i \frac{q-1}{\ell} \bar{\phi} \sigma \phi+\frac{q(2-q)}{\ell^{2}} \bar{\phi} \phi+\bar{F} F\right. \\
& \left.+\frac{i}{2} D_{\mu} \bar{\psi} \gamma^{\mu} \psi-\frac{i}{2} \bar{\psi} \gamma^{\mu} D_{\mu} \psi+i \bar{\psi} \sigma \psi-\frac{3 i}{4} \bar{\phi} \bar{\lambda} \psi+\frac{3 i}{4} \bar{\psi} \lambda \phi-\frac{2 q-1}{2 \ell} \bar{\psi} \psi\right) \\
& +i \bar{\epsilon}^{\prime} \gamma^{\mu} \epsilon\left(\varepsilon_{\mu \nu \rho} D^{\nu} \bar{\phi} D^{\rho} \phi+\frac{1}{\ell} D_{\mu} \bar{\phi} \phi-\frac{1}{\ell} \bar{\phi} D_{\mu} \phi+\frac{i}{2} \varepsilon_{\mu \nu \rho} D^{\nu} \bar{\psi} \gamma^{\rho} \psi+\frac{i}{2} \varepsilon_{\mu \nu \rho} \bar{\psi} \gamma^{\rho} D^{\nu} \psi\right. \\
& \left.+\frac{1}{4} \bar{\phi} \bar{\lambda} \gamma_{\mu} \psi-\frac{1}{4} \bar{\psi} \gamma_{\mu} \lambda \phi-\frac{i}{\ell} \bar{\psi} \gamma_{\mu} \psi\right) \\
& -\frac{i}{4} \epsilon^{\prime} \gamma^{\mu} \epsilon \bar{\psi} \gamma_{\mu} \bar{\lambda} \phi+\frac{i}{4} \bar{\epsilon}^{\prime} \gamma^{\mu} \bar{\epsilon} \bar{\phi} \lambda \gamma_{\mu} \psi
\end{aligned}
$$


Note that there are no surface terms in this expression. The bosonic part of $\int \delta V_{\text {chiral }}$ can be written as

$$
\int \mathrm{d}^{3} x \sqrt{g}\left(\frac{1}{2}\left|D_{\mu} \phi-i \epsilon_{\mu \nu \rho} v^{\nu} D^{\rho} \phi+\frac{i}{l} v_{\mu} \phi\right|^{2}+\bar{\phi}\left(\sigma+i \frac{q-1}{l}\right)^{2} \phi+\frac{1}{2}\left|\frac{1}{l} \phi-i v^{\mu} D_{\mu} \phi\right|^{2}+\bar{F} F\right),
$$

which is positive definite after constant shift of $\sigma$ (and $D$ ). Then, the saddle point of this is at $\phi=0, F=0$. For other values of $\sigma$, it is reasonable to think that the partition function and other exact quantities are obtained by the analytic continuation of $\sigma$. Alternatively, we can regard this $\delta V_{\text {chiral }}$ as follows. If we use $\delta\left[\left(\delta^{\prime} \psi\right)^{\dagger} \psi+\bar{\psi}\left(\delta^{\prime} \bar{\psi}\right)^{\dagger}\right]$, which is manifestly positive definite, as the $\delta$-exact terms for the matter multiplet, we find that the saddle point equations are $\delta \psi=0$ and the saddle point is at $\phi=0, F=0$ partly because of the boundary conditions. Because the addition of $\delta\left[\bar{\phi} \delta^{\prime} \phi-\left(\delta^{\prime} \bar{\phi}\right) \phi\right]$ will not change the 1-loop factor of the $\delta$-exact terms, we can think the saddle point of the $\int \delta V_{\text {chiral }}$ for the matter multiplet is at $\phi=0, F=0$.

\subsection{One-loop determinant}

\subsubsection{Vector multiplet}

We will compute the one-loop determinant at the saddle point of $\int \delta V_{\text {vector }}$.

With the addition of the term $t \delta V_{\text {vector }}$, we expand the fields around the saddle point as

$$
\begin{aligned}
A_{\theta} & =a_{\theta}+\frac{1}{\sqrt{t}} \hat{A}_{\theta}, \quad A_{\tilde{\mu}}=a_{\tilde{\mu}}+\frac{1}{\sqrt{t}} \hat{A}_{\tilde{\mu}} \\
\sigma & =\sigma_{0}+\frac{1}{\sqrt{t}} \hat{\sigma}, \quad D=-\frac{\sigma_{0}}{\ell}+\frac{1}{\sqrt{t}} \hat{D}, \\
\lambda & =\frac{1}{\sqrt{t}} \hat{\lambda}, \quad \bar{\lambda}=\frac{1}{\sqrt{t}} \hat{\bar{\lambda}} .
\end{aligned}
$$

where $a_{\tilde{\mu}}$ and $\sigma_{0}$ are boundary values in (2.16) and (2.17), and $a_{\theta}=0$. We should set $a_{\chi}=0$ because of the smoothness at $\theta=0$. On the other hand, $a_{\varphi}$ can be non-zero as an Wilson loop of the non-trivial 1-cycle of the manifold with the boundary except for $\theta_{0}=\pi / 2$.

For the gauge fixing, we will take the following Lorenz-like one:

$$
D_{\mu}^{(a)} \hat{A}^{\mu}=0, \quad\left(D_{\mu}^{(a)}=\nabla_{\mu}-i\left[a_{\mu}, \cdot\right]\right) .
$$

The boundary conditions for the fluctuation fields at $\theta=\theta_{0}$ are

$$
\hat{A}_{\tilde{\mu}}|=0, \quad \hat{\sigma}|=0
$$


With the gauge condition, we have

$$
\begin{aligned}
t \int \mathrm{d}^{3} x \sqrt{g} \mathcal{L}_{\mathrm{YM}}=\int \mathrm{d}^{3} x \sqrt{g} \operatorname{Tr}( & -\frac{1}{2} A_{\mu} D_{\nu}^{(a)} D^{(a) \nu} A^{\mu}+\frac{1}{\ell^{2}} A_{\mu} A^{\mu}-\frac{1}{2}\left[\sigma_{0}, A_{\mu}\right]^{2} \\
& -\frac{1}{2} \sigma D_{\mu}^{(a)} D^{(a) \mu} \sigma+(D+\sigma / \ell)^{2} \\
& \left.+\frac{i}{4} \bar{\lambda} \gamma^{\mu} D_{\mu}^{(a)} \lambda+\frac{i}{4} \lambda \gamma^{\mu} D_{\mu}^{(a)} \bar{\lambda}+\frac{i}{2} \bar{\lambda}\left[\sigma_{0}, \lambda\right]-\frac{1}{4 \ell} \bar{\lambda} \lambda\right) \\
& +\mathcal{O}\left(t^{-1 / 2}\right),
\end{aligned}
$$

where we have omitted the hat symbols for the fluctuation fields, for example $\hat{\sigma} \rightarrow \sigma$, for notational simplicity.

Following the arguments in [10] for the squashed (ellipsoid) $S^{3}$, we only consider $A_{\mu}$ with the gauge conditions and the fermions $\lambda, \bar{\lambda}$. The (quadratic) kinetic operators for them are

$$
L_{2}^{A}=\frac{1}{2} A_{\mu} \Delta_{A} A^{\mu}
$$

where

$$
\Delta_{A} \equiv-D_{\nu}^{(a)} D^{(a) \nu}+\frac{2}{\ell^{2}}+\left(\sigma_{0}^{\mathrm{adj}}\right)^{2}
$$

and

$$
L_{2}^{(\lambda, \bar{\lambda})}=\frac{1}{4} \operatorname{Tr}\left(\begin{array}{ll}
\lambda & \bar{\lambda}
\end{array}\right)\left(\begin{array}{cc}
0 & \Delta_{\bar{\lambda}} \\
\Delta_{\lambda} & 0
\end{array}\right)\left(\begin{array}{l}
\lambda \\
\bar{\lambda}
\end{array}\right),
$$

where

$$
\begin{aligned}
& \Delta_{\lambda} \equiv i \gamma^{\mu} D_{\mu}^{(a)}+i \sigma_{0}^{\mathrm{adj}}-\frac{1}{2 \ell}, \\
& \Delta_{\bar{\lambda}} \equiv i \gamma^{\mu} D_{\mu}^{(a)}-i \sigma_{0}^{\mathrm{adj}}-\frac{1}{2 \ell} .
\end{aligned}
$$

Note that we need to consider the eigenmodes for the pairs of $\lambda$ and $\bar{\lambda}$ because the boundary conditions identify $\lambda$ and $\bar{\lambda}$ at $\theta=\theta_{0}$, thus they are not independent fields.

The 1-loop factor for the bosonic modes is given by $\prod_{i} M_{i}^{-1}$ up to an irrelevant numerical factor where the eigenmode $A_{i}^{\mu}$ with the eigenvalue $M_{i}^{2}$ is defined by ${ }^{7}$

$$
\Delta_{A} A_{i}^{\mu}=M_{i}^{2} A_{i}^{\mu}, \quad \text { with } D_{\mu}^{(a)} A^{\mu}=0,
$$

\footnotetext{
7 More precisely, the eigenvalue problem is

$$
\int_{M} A_{\mu}^{\prime}\left(\Delta_{A}-M_{i}^{2}\right) A_{i}^{\mu}=0
$$

where $A_{\mu}^{\prime}$ is an arbitrary gauge field with the boundary condition and the gauge condition. Because $A^{\prime}$ can take an arbitrary value with the gauge condition in the bulk, $\int_{M} A_{\mu}^{\prime} A^{\mu}=0$ may mean $A^{\mu}=0$. Thus, this eigenvalue problem may be same as (2.73).
} 
where $A_{i}^{\mu}$ should satisfy the boundary condition. Note that $M_{i}^{2}$ is real and $M_{i}^{2}>\left(\sigma_{0}^{\text {adj }}\right)^{2}$.

For the fermions, the 1-loop factor is given by $\sqrt{\prod_{i} \nu_{i}}$ up to a numerical factor where the eigenmode with the eigenvalue $\nu_{i}$ is given by 8

$$
\left(\begin{array}{cc}
0 & C \Delta_{\bar{\lambda}} \\
C \Delta_{\lambda} & 0
\end{array}\right)\left(\begin{array}{l}
\lambda_{i} \\
\bar{\lambda}_{i}
\end{array}\right)=\nu_{i}\left(\begin{array}{l}
\lambda_{i} \\
\bar{\lambda}_{i}
\end{array}\right)
$$

which is the eigenvalue problem for the anti-symmetric operator although we will not use this operator. Instead of this, we will consider the following eigenvalue problem:

$$
\left(\begin{array}{cc}
0 & \Delta_{\bar{\lambda}} \\
\Delta_{\lambda} & 0
\end{array}\right)\left(\begin{array}{l}
\lambda_{i} \\
\bar{\lambda}_{i}
\end{array}\right)=\nu_{i}\left(\begin{array}{cc}
0 & -1 \\
1 & 0
\end{array}\right)\left(\begin{array}{l}
\lambda_{i} \\
\bar{\lambda}_{i}
\end{array}\right),
$$

where the determinant of this operator and the original one are clearly same. Thus the 1-loop factor for the fermions is also given by the eigenvalues of this operator as $\sqrt{\prod_{i} \nu_{i}}$, up to a numerical factor.

Now, basically following [10], we will show that almost all the eigenvalues in the bosonic and the fermionic 1-loop factors are canceled. Let us suppose $A_{\mu}$ be a bosonic eigenmode,

$$
\Delta_{A} A_{\mu}=M^{2} A_{\mu}, \quad D_{\mu}^{(a)} A^{\mu}=0 .
$$

Then, the following two $\lambda$ modes

$$
\lambda_{1} \equiv \gamma^{\mu} \epsilon A_{\mu}, \quad \lambda_{2} \equiv-\varepsilon^{\mu \nu \rho} \gamma_{\rho} \epsilon D_{\mu}^{(a)} A_{\nu}
$$

satisfy

$$
\Delta_{\lambda}\left(\begin{array}{l}
\lambda_{1} \\
\lambda_{2}
\end{array}\right)=\left(\begin{array}{cc}
i \sigma_{0}^{\text {adj }} & 1 \\
M^{2}-\left(\sigma_{0}^{\text {adj }}\right)^{2} & i \sigma_{0}^{\text {adj }}
\end{array}\right)\left(\begin{array}{l}
\lambda_{1} \\
\lambda_{2}
\end{array}\right) .
$$

Then, the following linear combinations of them,

$$
\lambda_{ \pm} \equiv \pm \sqrt{M^{2}-\left(\sigma_{0}^{\mathrm{adj}}\right)^{2}} \lambda_{1}+\lambda_{2}
$$

satisfy

$$
\Delta_{\lambda} \lambda_{ \pm}=\nu_{ \pm} \lambda_{ \pm} \quad\left(\nu_{ \pm}=i \sigma_{0}^{\text {adj }} \pm \sqrt{M^{2}-\left(\sigma_{0}^{\text {adj }}\right)^{2}}\right)
$$

Similarly, the following two $\bar{\lambda}$ modes

$$
\bar{\lambda}_{1} \equiv-\gamma^{\mu} \bar{\epsilon} A_{\mu}, \quad \bar{\lambda}_{2} \equiv-\varepsilon^{\mu \nu \rho} \gamma_{\rho} \bar{\epsilon} D_{\mu}^{(a)} A_{\nu}
$$

\footnotetext{
${ }^{8}$ The same argument as the bosonic modes can be applied for the fermions.
} 
satisfy

$$
\Delta_{\bar{\lambda}}\left(\begin{array}{l}
\bar{\lambda}_{1} \\
\bar{\lambda}_{2}
\end{array}\right)=\left(\begin{array}{cc}
-i \sigma_{0}^{\mathrm{adj}} & -1 \\
-M^{2}+\left(\sigma_{0}^{\mathrm{adj}}\right)^{2} & -i \sigma_{0}^{\mathrm{adj}}
\end{array}\right)\left(\begin{array}{l}
\bar{\lambda}_{1} \\
\bar{\lambda}_{2}
\end{array}\right)
$$

and the linear combinations of them

$$
\bar{\lambda}_{ \pm} \equiv \pm \sqrt{M^{2}-\left(\sigma_{0}^{\mathrm{adj}}\right)^{2}} \bar{\lambda}_{1}+\bar{\lambda}_{2}
$$

satisfy

$$
\Delta_{\bar{\lambda}} \bar{\lambda}_{ \pm}=\bar{\nu}_{ \pm} \bar{\lambda}_{ \pm} \quad\left(\bar{\nu}_{ \pm}=-i \sigma_{0}^{\text {adj }} \mp \sqrt{M^{2}-\left(\sigma_{0}^{\text {adj }}\right)^{2}}=-\nu_{ \pm}\right)
$$

Therefore, we have two fermionic eigenmodes: $\left(\begin{array}{l}\lambda_{+} \\ \bar{\lambda}_{+}\end{array}\right)$and $\left(\begin{array}{l}\lambda_{-} \\ \bar{\lambda}_{-}\end{array}\right)$whose eigenvalues are $\nu_{+}$and $\nu_{-}=-\nu_{+}^{*}$. We can show that these modes satisfy the boundary condition

$$
\ell e^{i(\varphi-\chi)} \gamma^{\theta} \lambda_{ \pm}\left|=\bar{\lambda}_{ \pm}\right|
$$

where we have used the fact that $A_{\mu}$ satisfies the boundary condition $\left(A_{\tilde{\mu}} \mid=0\right)$. The contribution of these two fermion modes is canceled by the corresponding bosonic eigenmode because $\sqrt{-\nu_{+} \nu_{-}}=M$.

Conversely, let us suppose that $\left(\begin{array}{l}\lambda \\ \bar{\lambda}\end{array}\right)$ be a fermionic eigenmode with,

$$
\Delta_{\lambda} \lambda=\nu \lambda, \quad \Delta_{\bar{\lambda}} \bar{\lambda}=-\nu \bar{\lambda}
$$

which satisfies the boundary condition $\ell e^{i(\varphi-\chi)} \gamma^{\theta} \lambda|=\bar{\lambda}|$. Then we can construct a bosonic eigenmode

$$
A_{\mu} \equiv\left(\nu-i \sigma_{0}^{\mathrm{adj}}\right)\left(\bar{\epsilon} \gamma_{\mu} \lambda+\epsilon \gamma_{\mu} \bar{\lambda}\right)-i D_{\mu}^{(a)}(\bar{\epsilon} \lambda-\epsilon \bar{\lambda})
$$

which satisfies the boundary condition $\left(A_{\tilde{\mu}} \mid=0\right)$ and the gauge fixing condition $\left(D_{\mu}^{(a)} A^{\mu}=\right.$ $0)$. The corresponding eigenvalue is $M^{\prime 2}=\left(\nu-i \sigma_{0}^{\text {adj }}\right)^{2}+\left(\sigma_{0}^{\text {adj }}\right)^{2}$, i.e.

$$
\Delta_{A} A_{\mu}=\left[\left(\nu-i \sigma_{0}^{\text {adj }}\right)^{2}+\left(\sigma_{0}^{\text {adj }}\right)^{2}\right] A_{\mu} .
$$

Because $M^{\prime 2}$ should be real and $M^{2}>\left(\sigma_{0}^{\text {adj }}\right)^{2}$, we have $\nu=i \sigma_{0}^{\text {adj }} \pm \sqrt{M^{\prime 2}-\left(\sigma_{0}^{\text {adj }}\right)^{2}}$ and $-\nu^{*}=i \sigma_{0}^{\text {adj }} \mp \sqrt{M^{\prime 2}-\left(\sigma_{0}^{\text {adj }}\right)^{2}}$. Therefore the 1-loop contributions of $\left(\begin{array}{l}\lambda \\ \bar{\lambda}\end{array}\right)$ and the fermionic mode corresponding the eigenvalue $-\nu^{*}$ are canceled by the one from the bosonic eigenmode. 


\section{Unpaired eigenmodes}

We have shown that there is a map between the bosonic and fermionic eigenmodes and almost all the eigenvalues are canceled in evaluating the 1-loop factor. Hence, we may study only the bosonic (fermionic) eigenmodes whose eigenvalues are not canceled by the fermionic (bosonic) eigenvalues. We will call such modes as the unpaired bosonic (fermionic) eigenmodes. In Appendix A, we summarize some useful formulas for Killing spinors and Killing vectors (2.24), (2.25), (2.26), which will be used in the following calculation.

The unpaired bosonic eigenmodes should satisfy $\left(\begin{array}{l}\lambda_{+} \\ \bar{\lambda}_{+}\end{array}\right)=0$ or $\left(\begin{array}{l}\lambda_{-} \\ \bar{\lambda}_{-}\end{array}\right)=0$, where $\lambda_{ \pm}, \bar{\lambda}_{ \pm}$ are the corresponding fermionic modes, as we can see from the discussions of the cancellations. Any unpaired bosonic mode can be written using the general decomposition of the gauge field:

$$
A_{\mu} \equiv v_{\mu} Y+v_{\mu}^{+} Y_{+}+v_{\mu}^{-} Y_{-}=v_{\mu}^{X} Y_{X}
$$

where $v_{\mu}^{X}(X=$ no mark,,+-$)$ are defined by (2.24) $-(2.26)$. The boundary conditions can be rewritten into

$$
Y\left|=0, \quad\left(e^{-i(\varphi-\chi)} Y_{+}+e^{i(\varphi-\chi)} Y_{-}\right)\right|=0
$$

and the gauge fixing condition, $D_{\mu}^{(a)} A^{\mu}=0$, becomes

$$
v_{X}^{\mu} D_{\mu}^{(a)} Y_{X}=0
$$

where we have used $D_{\mu}^{(a)} A_{\nu}=v_{\nu}^{X} D_{\mu}^{(a)} Y_{X}+\frac{1}{\ell} \varepsilon_{\mu \nu \rho} v_{X}^{\rho} Y_{X} Y$. The eigenvalue equation, $\Delta_{A} A_{\mu}=$ $M^{2} A_{\mu}$, can be written as

$$
-v_{\mu}^{X} D^{(a) \nu} D_{\nu}^{(a)} Y_{X}+\frac{2}{\ell} \varepsilon_{\mu \nu \rho} v_{X}^{\rho} D^{(a) \nu} Y_{X}=\left[M^{2}-\left(\sigma_{0}^{\mathrm{adj}}\right)^{2}-\frac{4}{\ell^{2}}\right] v_{\mu}^{X} Y_{X},
$$

which can be decomposed into the following three equations:

$$
\begin{aligned}
-D^{(a) \nu} D_{\nu}^{(a)} Y+\frac{2 i}{\ell} v_{+}^{\nu} D_{\nu}^{(a)} Y_{+}-\frac{2 i}{\ell} v_{-}^{\nu} D_{\nu}^{(a)} Y_{-} & =\left[M^{2}-\left(\sigma_{0}^{\text {adj }}\right)^{2}-\frac{4}{\ell^{2}}\right] Y, \\
-D^{(a) \nu} D_{\nu}^{(a)} Y_{-}+\frac{i}{\ell} v_{+}^{\nu} D_{\nu}^{(a)} Y+\frac{2 i}{\ell} v^{\nu} D_{\nu}^{(a)} Y_{-} & =\left[M^{2}-\left(\sigma_{0}^{\text {adj }}\right)^{2}-\frac{4}{\ell^{2}}\right] Y_{-}, \\
-D^{(a) \nu} D_{\nu}^{(a)} Y_{+}-\frac{i}{\ell} v_{-}^{\nu} D_{\nu}^{(a)} Y-\frac{2 i}{\ell} v^{\nu} D_{\nu}^{(a)} Y_{+} & =\left[M^{2}-\left(\sigma_{0}^{\text {adj }}\right)^{2}-\frac{4}{\ell^{2}}\right] Y_{+} .
\end{aligned}
$$


The unpaired bosonic modes should satisfy $C \lambda_{1}+\lambda_{2}=0$, more explicitly, $\left(C-\frac{2}{\ell}\right)(Y \epsilon+$ $\left.2 Y_{-} \bar{\epsilon}\right)+i\left(\gamma^{\mu} \epsilon D_{\mu}^{(a)} Y+2 \gamma^{\mu} \bar{\epsilon} D_{\mu}^{(a)} Y_{-}\right)=0$ which is equivalent to

$$
\begin{aligned}
-2\left(C-\frac{2}{\ell}\right) Y_{-}+i v_{+}^{\mu} D_{\mu}^{(a)} Y+2 i v^{\mu} D_{\mu}^{(a)} Y_{-} & =0, \\
\left(C-\frac{2}{\ell}\right) Y+i v^{\mu} D_{\mu}^{(a)} Y+2 i v_{-}^{\mu} D_{\mu}^{(a)} Y_{-} & =0,
\end{aligned}
$$

where we have used

$$
\begin{aligned}
& \lambda_{1}=\gamma^{\mu} \epsilon A_{\mu}=Y \epsilon+2 Y_{-} \bar{\epsilon} \\
& \lambda_{2}=-\varepsilon^{\mu \nu \rho} \gamma_{\rho} \epsilon D_{\mu}^{(a)} A_{\nu}=-\frac{2}{\ell}\left(Y \epsilon+2 Y_{-} \bar{\epsilon}\right)+i\left(\gamma^{\mu} \epsilon D_{\mu}^{(a)} Y+2 \gamma^{\mu} \bar{\epsilon} D_{\mu}^{(a)} Y_{-}\right), \\
& \bar{\lambda}_{1}=-\gamma^{\mu} \bar{\epsilon} A_{\mu}=Y \bar{\epsilon}+2 Y_{+} \epsilon \\
& \bar{\lambda}_{2}=-\varepsilon^{\mu \nu \rho} \gamma_{\rho} \bar{\epsilon} D_{\mu}^{(a)} A_{\nu}=\frac{2}{\ell}\left(Y \bar{\epsilon}+2 Y_{+} \epsilon\right)-i\left(\gamma^{\mu} \bar{\epsilon} D_{\mu}^{(a)} Y+2 \gamma^{\mu} \epsilon D_{\mu}^{(a)} Y_{+}\right) .
\end{aligned}
$$

and defined

$$
C \equiv \pm \sqrt{M^{2}-\left(\sigma_{0}^{\text {adj }}\right)^{2}}
$$

For the other condition, $C \bar{\lambda}_{1}+\bar{\lambda}_{2}=0$, we have

$$
\left(C+\frac{2}{\ell}\right)\left(Y \bar{\epsilon}+2 Y_{+} \epsilon\right)-i\left(\gamma^{\mu} \bar{\epsilon} D_{\mu}^{(a)} Y+2 \gamma^{\mu} \epsilon D_{\mu}^{(a)} Y_{+}\right)=0
$$

which is equivalent to

$$
\begin{aligned}
& 2\left(C+\frac{2}{\ell}\right) Y_{+}-i v_{-}^{\mu} D_{\mu}^{(a)} Y-2 i v^{\mu} D_{\mu}^{(a)} Y_{+}=0 \\
& -\left(C+\frac{2}{\ell}\right) Y-i v^{\mu} D_{\mu}^{(a)} Y-2 i v_{+}^{\mu} D_{\mu}^{(a)} Y_{+}=0 .
\end{aligned}
$$

Now we found all the equations for the unpaired bosonic modes and will solve them. From (2.91), (2.97) and (2.105), we obtain

$$
C Y=0 \text {. }
$$

We can easily see that there are no nontrivial solutions if $C=0$, which implies $\lambda_{2}=\bar{\lambda}_{2}=0$ and then $\varepsilon^{\mu \nu \rho} D_{\mu}^{(a)} A_{\nu}=0$. Therefore, we will solve them for $Y=0$. This implies

$$
\left(C-\frac{2}{\ell}\right) Y_{-}-i v^{\mu} D_{\mu}^{(a)} Y_{-}=0, \quad v_{-}^{\mu} D_{\mu}^{(a)} Y_{-}=0
$$

and

$$
\left(C+\frac{2}{\ell}\right) Y_{+}-i v^{\mu} D_{\mu}^{(a)} Y_{+}=0, \quad v_{+}^{\mu} D_{\mu}^{(a)} Y_{+}=0
$$


Using the boundary conditions, we take the following ansatz:

$$
Y_{-}=f_{-}(\theta) e^{i((m-1) \varphi-(n-1) \chi)}, \quad Y_{+}=f_{+}(\theta) e^{i((m+1) \varphi-(n+1) \chi)},
$$

where $m$ and $n$ are integers. Then, the $v_{\mp}^{\mu} D_{\mu}^{(a)} Y_{\mp}=0$ is solved as

$$
f_{-}(\theta) \propto \cos ^{m-1-a_{\varphi}} \theta \sin ^{n-1} \theta, \quad f_{+}(\theta) \propto \cos ^{-m-1+a_{\varphi}} \theta \sin ^{-n-1} \theta .
$$

Because of the regularity at $\theta=0$, we have two cases: $f_{+}=0, n \geq 1$ or $f_{-}=0, n \leq-1$, however, the boundary conditions (2.90) do not allow both cases for $\theta_{0}<\pi / 2$.

Therefore, we conclude that there is no bosonic unpaired eigenmode for $\theta_{0}<\pi / 2$. For the special case that $\theta_{0}=\pi / 2$, there are the following unpaired bosonic eigenmodes:

$$
\begin{aligned}
& Y=Y_{+}=0, \quad Y_{-} \propto \cos ^{m-1} \theta \sin ^{n-1} \theta e^{i(m-1) \varphi-i(n-1) \chi}, \\
& C=\frac{m+n}{\ell}>0 \quad(m \geq 2, n \geq 1)
\end{aligned}
$$

or

$$
\begin{aligned}
& Y=Y_{-}=0, \quad Y_{+} \propto \cos ^{-m-1} \theta \sin ^{-n-1} \theta e^{i(m+1) \varphi-i(n+1) \chi} \\
& C=\frac{m+n}{\ell}<0 \quad(m \leq-2, n \leq-1) .
\end{aligned}
$$

For $S^{3}$, i.e. the case without boundary, the unpaired bosonic eigenmodes are given by

$$
\begin{aligned}
& Y=Y_{+}=0, \quad Y_{-} \propto \cos ^{m-1} \theta \sin ^{n-1} \theta e^{i(m-1) \varphi-i(n-1) \chi}, \\
& C=\frac{m+n}{\ell}>0 \quad(m \geq 1, n \geq 1)
\end{aligned}
$$

or

$$
\begin{aligned}
& Y=Y_{-}=0, \quad Y_{+} \propto \cos ^{-m-1} \theta \sin ^{-n-1} \theta e^{i(m+1) \varphi-i(n+1) \chi} \\
& C=\frac{m+n}{\ell}<0 \quad(m \leq-1, n \leq-1)
\end{aligned}
$$

Let us consider unpaired fermionic eigenmodes,

$$
\Delta_{\lambda} \lambda=\nu \lambda, \quad \Delta_{\bar{\lambda}} \bar{\lambda}=-\nu \bar{\lambda}
$$

for which the corresponding bosonic eigenmode vanishes, i.e.

$$
\left(\nu-i \sigma_{0}^{\mathrm{adj}}\right)\left(\bar{\epsilon} \gamma_{\mu} \lambda+\epsilon \gamma_{\mu} \bar{\lambda}\right)-i D_{\mu}^{(a)}(\bar{\epsilon} \lambda-\epsilon \bar{\lambda})=0 .
$$


We can expand $\lambda$ and $\bar{\lambda}$ as

$$
\lambda=\Lambda \epsilon+\Lambda^{\prime} \bar{\epsilon}, \quad \bar{\lambda}=\bar{\Lambda} \bar{\epsilon}+\bar{\Lambda}^{\prime} \epsilon,
$$

where $\Lambda, \Lambda^{\prime}, \bar{\Lambda}$ and $\bar{\Lambda}^{\prime}$ are scalars. Then the boundary conditions can be written as

$$
\Lambda|+\bar{\Lambda}|=0, \quad e^{i(\varphi-\chi)} \Lambda^{\prime}\left|+e^{-i(\varphi-\chi)} \bar{\Lambda}^{\prime}\right|=0 .
$$

In this expansion, the eigenvalue equation, $\Delta_{\lambda} \lambda=\nu \lambda$, is equivalent to

$$
\begin{aligned}
& i v_{+}^{\mu} D_{\mu}^{(a)} \Lambda+i v^{\mu} D_{\mu}^{(a)} \Lambda^{\prime}=-\left(\nu-i \sigma_{0}^{\mathrm{adj}}+\frac{2}{\ell}\right) \Lambda^{\prime}, \\
& i v^{\mu} D_{\mu}^{(a)} \Lambda+i v_{-}^{\mu} D_{\mu}^{(a)} \Lambda^{\prime}=\left(\nu-i \sigma_{0}^{\text {adj }}+\frac{2}{\ell}\right) \Lambda,
\end{aligned}
$$

and $\Delta_{\bar{\lambda}} \bar{\lambda}=-\nu \bar{\lambda}$ is equivalent to

$$
\begin{aligned}
& i v^{\mu} D_{\mu}^{(a)} \bar{\Lambda}+i v_{+}^{\mu} D_{\mu}^{(a)} \bar{\Lambda}^{\prime}=\left(\nu-i \sigma_{0}^{\mathrm{adj}}-\frac{2}{\ell}\right) \bar{\Lambda} \\
& i v_{-}^{\mu} D_{\mu}^{(a)} \bar{\Lambda}+i v^{\mu} D_{\mu}^{(a)} \bar{\Lambda}^{\prime}=-\left(\nu-i \sigma_{0}^{\mathrm{adj}}-\frac{2}{\ell}\right) \bar{\Lambda}^{\prime} .
\end{aligned}
$$

The equation (2.116) can be put into the form

$$
\begin{aligned}
& i v^{\mu} D_{\mu}^{(a)}(\Lambda+\bar{\Lambda})=\left(\nu-i \sigma_{0}^{\mathrm{adj}}\right)(\Lambda+\bar{\Lambda}), \\
& i v_{+}^{\mu} D_{\mu}^{(a)}(\Lambda+\bar{\Lambda})=-2\left(\nu-i \sigma_{0}^{\mathrm{adj}}\right) \Lambda^{\prime}, \\
& i v_{-}^{\mu} D_{\mu}^{(a)}(\Lambda+\bar{\Lambda})=-2\left(\nu-i \sigma_{0}^{\mathrm{adj}}\right) \bar{\Lambda}^{\prime} .
\end{aligned}
$$

We will solve the equations above for the unpaired fermionic modes. By (2.119), (2.123) and (2.124), we obtain

$$
v_{+}^{\mu} D_{\mu}^{(a)} \bar{\Lambda}=0,
$$

and by (2.122), (2.123) and (2.125), we obtain

$$
v_{-}^{\mu} D_{\mu}^{(a)} \Lambda=0 .
$$

If we make the ansatz,

$$
\Lambda=f(\theta) e^{i(m \varphi-n \chi)}, \quad \bar{\Lambda}=\bar{f}(\theta) e^{i(m \varphi-n \chi)},
$$

where $m$ and $n$ are integers, then we can solve (2.126) and (2.127) as

$$
f(\theta) \propto \cos ^{m-a_{\varphi}} \theta \sin ^{n} \theta, \quad \bar{f}(\theta) \propto \cos ^{-m+a_{\varphi}} \theta \sin ^{-n} \theta .
$$


For $\theta_{0}<\pi / 2$, the boundary conditions and the regularity at $\theta=0$ fix $f, \bar{f}$ as

$$
f(\theta)=f_{0}\left(\frac{\cos \theta}{\cos \theta_{0}}\right)^{m-a_{\varphi}}, \quad \bar{f}(\theta)=-f_{0}\left(\frac{\cos \theta}{\cos \theta_{0}}\right)^{-m+a_{\varphi}},
$$

where $f_{0}$ is a constant. Then eq. (2.123) determines the eigenvalue as

$$
\nu=i \sigma_{0}^{\mathrm{adj}}+\frac{m-a_{\varphi}}{\ell} .
$$

Furthermore, from (2.124) and (2.125) $, \Lambda^{\prime}, \bar{\Lambda}^{\prime}$ should take the following forms

$$
\begin{aligned}
& \Lambda^{\prime}=i f_{0} \frac{\sin \theta}{\cos \theta}\left(\frac{\cos \theta}{\cos \theta_{0}}\right)^{m-a_{\varphi}} e^{i(m-1) \varphi+i \chi}, \\
& \bar{\Lambda}^{\prime}=-i f_{0} \frac{\sin \theta}{\cos \theta}\left(\frac{\cos \theta}{\cos \theta_{0}}\right)^{-m+a_{\varphi}} e^{i(m+1) \varphi-i \chi},
\end{aligned}
$$

which are consistent with the boundary conditions. We can also check that these satisfy (2.120) and (2.121). Thus, these solutions are indeed the fermionic unpaired eigenmodes for $\theta_{0}<\pi / 2$.

For $\theta_{0}=\pi / 2.9$ we find that the followings are the consistent solutions:

$$
\begin{aligned}
\Lambda & =f_{0} \cos ^{m} \theta \sin ^{n} \theta e^{i(m \varphi-n \chi)}, \quad \bar{\Lambda}=0, \\
\Lambda^{\prime} & =\frac{i f_{0}}{m+n}\left(m \cos ^{m-1} \theta \sin ^{n+1} \theta-n \cos ^{m+1} \theta \sin ^{n-1} \theta\right) e^{i(m-1) \varphi-i(n-1) \chi}, \quad \bar{\Lambda}^{\prime}=0, \\
\nu & =i \sigma_{0}^{\text {adj }}+\frac{m+n}{\ell} \quad(m \geq 2, n \geq 1),
\end{aligned}
$$

and

$$
\begin{aligned}
\Lambda & =0, \quad \bar{\Lambda}=f_{0} \cos ^{-m} \theta \sin ^{-n} \theta e^{i(m \varphi-n \chi)} \\
\Lambda^{\prime} & =0, \quad \bar{\Lambda}^{\prime}=\frac{i f_{0}}{m+n}\left(m \cos ^{-m-1} \theta \sin ^{-n+1} \theta-n \cos ^{-m+1} \theta \sin ^{-n-1} \theta\right) e^{i(m+1) \varphi-i(n+1) \chi} \\
\nu & =i \sigma_{0}^{\text {adj }}+\frac{m+n}{\ell} \quad(m \leq-2, n \leq-1) .
\end{aligned}
$$

For $S^{3}$, the fermionic unpaired eigenmodes are given by

$$
\begin{aligned}
\Lambda & =f_{0} \cos ^{m} \theta \sin ^{n} \theta e^{i(m \varphi-n \chi)}, \quad \bar{\Lambda}=0, \\
\Lambda^{\prime} & =\frac{i f_{0}}{m+n}\left(m \cos ^{m-1} \theta \sin ^{n+1} \theta-n \cos ^{m+1} \theta \sin ^{n-1} \theta\right) e^{i(m-1) \varphi-i(n-1) \chi}, \quad \bar{\Lambda}^{\prime}=0, \\
\nu & =i \sigma_{0}^{\mathrm{adj}}+\frac{m+n}{\ell} \quad(m \geq 0, n \geq 0, m n \neq 0),
\end{aligned}
$$

\footnotetext{
${ }^{9}$ Note that $a_{\varphi}=a_{\chi}=0$ in this case.
} 
and

$$
\begin{aligned}
\Lambda & =0, \quad \bar{\Lambda}=f_{0} \cos ^{-m} \theta \sin ^{-n} \theta e^{i(m \varphi-n \chi)} \\
\Lambda^{\prime} & =0, \quad \bar{\Lambda}^{\prime}=\frac{i f_{0}}{m+n}\left(m \cos ^{-m-1} \theta \sin ^{-n+1} \theta-n \cos ^{-m+1} \theta \sin ^{-n-1} \theta\right) e^{i(m+1) \varphi-i(n+1) \chi} \\
\nu & =i \sigma_{0}^{\text {adj }}+\frac{m+n}{\ell} \quad(m \leq 0, n \leq 0, m n \neq 0)
\end{aligned}
$$

Therefore, up to an overall numerical factor 10 the 1-loop factor for the vector multiplet for $\theta_{0}<\pi / 2$ is given by

$$
Z_{\text {vector }}^{1-\text { loop }}=\prod_{\alpha \in \Delta_{+}} \prod_{m}\left(i \alpha\left(\sigma_{0}\right) \ell+m-\alpha\left(a_{\varphi}\right)\right)
$$

where $\Delta_{+}$is the set of the positive roots. For the special value $\theta_{0}=\pi / 2$, the 1-loop factor is

$$
\begin{aligned}
Z_{\text {vector }}^{1-\text { loop }} & =\prod_{\alpha \in \Delta_{+}} \frac{\prod_{|m| \geq 2,|n| \geq 1, m n>0}\left(i \alpha\left(\sigma_{0}\right) \ell-m-n\right)\left(i \alpha\left(\sigma_{0}\right) \ell+m+n\right)}{\prod_{|m| \geq 2,|n| \geq 1, m n>0}\left(\alpha\left(\sigma_{0}\right)^{2} \ell^{2}+(m+n)^{2}\right)} \\
& =1 .
\end{aligned}
$$

For $S^{3}$, the 1-loop factor is given by

$$
\begin{aligned}
Z_{\text {vector }}^{1-\text { loop }} & =\prod_{\alpha \in \Delta_{+}} \frac{\prod_{|m| \geq 1,|n| \geq 1, m n>0}\left(i \alpha\left(\sigma_{0}\right) \ell-m-n\right) \prod_{|m| \geq 0,|n| \geq 0, m n>0}\left(i \alpha\left(\sigma_{0}\right) \ell+m+n\right)}{\prod_{|m| \geq 1,|n| \geq 1, m n>0}\left(\alpha\left(\sigma_{0}\right)^{2} \ell^{2}+(m+n)^{2}\right)} \\
& =\prod_{\alpha \in \Delta_{+}} \frac{1}{\alpha\left(\sigma_{0}\right)^{2} \ell^{2}} \prod_{m \geq 0, n \geq 0} \frac{\left(\alpha\left(\sigma_{0}\right)^{2} \ell^{2}+(m+n)^{2}\right)}{\left(\alpha\left(\sigma_{0}\right)^{2} \ell^{2}+(m+n+2)^{2}\right)} \\
& =\prod_{\alpha \in \Delta_{+}} \prod_{m \geq 1}\left(\alpha\left(\sigma_{0}\right)^{2} \ell^{2}+m^{2}\right)^{2},
\end{aligned}
$$

which, of course, coincides with the one obtained in [7, 10].

\subsubsection{Chiral multiplet}

Next, let us consider the chiral multiplet. Expanding the fields around the saddle point and leaving only the quadratic terms, we have

$$
t \int \mathrm{d}^{3} x \sqrt{g} \delta V_{\text {chiral }}=\int \mathrm{d}^{3} x \sqrt{g} \mathcal{L}_{\text {reg }}+\mathcal{O}\left(t^{-1 / 2}\right),
$$

\footnotetext{
${ }^{10}$ We have also neglected a factor like $\ell^{p}$ where $p$ is a number.
} 
where

$$
\begin{aligned}
\mathcal{L}_{\mathrm{reg}} & =\bar{\phi} \Delta_{\phi} \phi+\bar{\psi} \Delta_{\psi} \psi \\
\Delta_{\phi} & =-D_{\mu}^{(a)} D^{(a) \mu}+\sigma_{0}^{2}+2 i \frac{q-1}{\ell} \sigma_{0}+\frac{q(2-q)}{\ell^{2}} \\
\Delta_{\psi} & =-i \gamma^{\mu} D_{\mu}^{(a)}+i \sigma_{0}-\frac{2 q-1}{2 \ell}
\end{aligned}
$$

Hereafter, we set

$$
\omega=i \sigma_{0}-(q-1) / \ell
$$

The eigenvalue problems for the 1-loop factor are $\Delta_{\phi} \phi=M^{2} \phi$ and $\Delta_{\psi} \psi=\nu \psi$. Note that the boundary conditions for $\psi$ and $\bar{\psi}$ are independently imposed, thus we can consider the eigenvalue problems independently. For $\bar{\psi}$, we have $\Delta_{\psi} \bar{\psi}=\nu \bar{\psi}$ where $\sigma_{0}$ in $\Delta_{\psi}$ are in the complex conjugate representation of the one for $\psi$.

The cancellations in the 1-loop factor between the contributions from the bosonic and fermionic modes can be seen as follows 11

Let us suppose $\psi$ as a fermionic eigenmode: $\Delta_{\psi} \psi=\nu \psi$ satisfying the boundary conditions (2.20). Then, if we define $\phi_{1} \equiv \bar{\epsilon} \psi$, we find that $\phi_{1}$ is a scalar eigenmode, i.e. $\Delta_{\phi} \phi_{1}=\nu(\nu-2 \omega) \phi_{1}$, which satisfies the the boundary condition.

Conversely, for a given scalar eigenmode $\left(\Delta_{\phi} \phi=M^{2} \phi\right)$ satisfying the boundary condition, we define

$$
\psi_{ \pm} \equiv\left(\nu_{ \pm}-\omega+\frac{1}{\ell}\right) \epsilon \phi-i \gamma^{\mu} \epsilon D_{\mu}^{(a)} \phi,
$$

where $\nu_{ \pm} \equiv \omega \pm \sqrt{M^{2}+\omega^{2}}$. Then, we find that

$$
\Delta_{\psi} \psi_{ \pm}=\nu_{ \pm} \psi_{ \pm}
$$

and $\psi_{ \pm}$satisfy the boundary condition.

Therefore, we may evaluate only the eigenvalues which are not canceled.

\section{Unpaired eigenmodes}

The unpaired fermionic eigenmode $\psi$ should satisfy $\phi_{1}(\equiv \bar{\epsilon} \psi)=0$. For such modes, we can take $\psi=\bar{\epsilon} \Psi$, where $\Psi$ is a scalar function on which any boundary condition is not

\footnotetext{
${ }^{11}$ The cancellations in the 1-loop factor can be seen more transparently as we will show in Appendix C.
} 
imposed. Since $\psi$ is a fermionic eigenmode, $\Delta_{\psi} \psi=\nu \psi$, we have

$$
\begin{aligned}
v_{-}^{\mu} D_{\mu}^{(a)} \Psi & =0, \\
i v^{\mu} D_{\mu}^{(a)} \Psi & =\left(\nu-\omega-\frac{1}{\ell}\right) \Psi .
\end{aligned}
$$

Therefore, we obtain

$$
\begin{aligned}
& \Psi \propto \cos ^{m-a_{\varphi}} \theta \sin ^{n} \theta e^{i m \varphi-i n \chi}, \\
& \nu=i \sigma_{0}-\frac{q-2}{\ell}+\frac{m-a_{\varphi}+n}{\ell} \quad(m \in \mathbb{Z}, n \geq 0),
\end{aligned}
$$

for $\theta_{0}<\pi / 2$, or

$$
\begin{aligned}
& \Psi \propto \cos ^{m} \theta \sin ^{n} \theta e^{i m \varphi-i n \chi}, \\
& \nu=i \sigma_{0}-\frac{q-2}{\ell}+\frac{m+n}{\ell} \quad(m \geq 0, n \geq 0),
\end{aligned}
$$

for $\theta_{0}=\pi / 2$ and for $S^{3}$.

On the other hand, the unpaired bosonic eigenmodes, $\Delta_{\phi} \phi=M^{2} \phi$, should satisfy

$$
\left(\nu-\omega+\frac{1}{\ell}\right) \epsilon \phi-i \gamma^{\mu} \epsilon D_{\mu}^{(a)} \phi=0 \quad\left(\nu(\nu-2 \omega)=M^{2}\right),
$$

which are equivalent to

$$
\begin{aligned}
v_{+}^{\mu} D_{\mu}^{(a)} \phi & =0, \\
i v^{\mu} D_{\mu}^{(a)} \phi & =\left(\nu-\omega+\frac{1}{\ell}\right) \phi .
\end{aligned}
$$

It can be easily checked that these equations imply $\Delta_{\phi} \phi=M^{2} \phi$.

For $\theta_{0}<\pi / 2$, we can show that there is no nontrivial solution which satisfies equation (2.163) and the boundary condition $\phi \mid=0$. For $\theta_{0}=\pi / 2$, we obtain the following solutions

$$
\begin{aligned}
& \phi \propto \cos ^{-m} \theta \sin ^{-n} \theta e^{i m \varphi-i n \chi} \\
& \nu=i \sigma_{0}-\frac{q}{\ell}+\frac{m+n}{\ell} \quad(m \leq-1, n \leq 0) .
\end{aligned}
$$

For $S^{3}$, we obtain

$$
\begin{aligned}
& \phi \propto \cos ^{-m} \theta \sin ^{-n} \theta e^{i m \varphi-i n \chi}, \\
& \nu=i \sigma_{0}-\frac{q}{\ell}+\frac{m+n}{\ell} \quad(m \leq 0, n \leq 0) .
\end{aligned}
$$


Therefore, up to an overall constant, the 1-loop determinants for the chiral multiplet are given by 12

$$
Z_{\text {chiral }}^{1-\text { loop }}=\prod_{\rho} \prod_{m} \prod_{n \geq 0}\left(i \rho\left(\sigma_{0}\right) \ell-q+2+m-\rho\left(a_{\varphi}\right)+n\right),
$$

in the case where $\theta_{0}<\pi / 2$, and

$$
Z_{\text {chiral }}^{1-\text { loop }}=\prod_{\rho} \frac{\prod_{m \geq 0, n \geq 0}\left(i \rho\left(\sigma_{0}\right) \ell-q+2+m+n\right)}{\prod_{m \geq 1, n \geq 0}\left(-i \rho\left(\sigma_{0}\right) \ell+q+m+n\right)},
$$

in the case where $\theta_{0}=\pi / 2$, and

$$
Z_{\text {chiral }}^{1-\text { loop }}=\prod_{\rho} \frac{\prod_{m \geq 0, n \geq 0}\left(i \rho\left(\sigma_{0}\right) \ell-q+2+m+n\right)}{\prod_{m \geq 0, n \geq 0}\left(-i \rho\left(\sigma_{0}\right) \ell+q+m+n\right)},
$$

in the case for $S^{3}$. The result for $S^{3}$ is same as the one in [7, 10].

\subsection{Partition functions and Wilson loops}

Combining the results obtained in this section, we find that the exact partition function for $\theta_{0}<\pi / 2\left(\right.$ or $\left.\theta_{0}=\pi / 2\right)$ is given by

$$
Z=Z_{\text {classical }} Z_{\text {vector }}^{1-\text { loo }} Z_{\text {chiral }}^{1-\text { loop }}
$$

where

$$
Z_{\text {classical }}=e^{\left(-i \frac{k}{2 \pi \ell} \operatorname{Tr}\left(\sigma_{0}\right)^{2}-\frac{2 i \zeta}{\pi \ell^{2}} \operatorname{Tr} \sigma_{0}\right) V\left(\theta_{0}\right)}
$$

where $V\left(\theta_{0}\right)=2 \pi^{2} \sin ^{2} \theta_{0} \ell^{3}$. Because $\sigma_{0}$ was fixed at the boundary, there is no matrix integral unlike the case without a boundary.

The supersymmetric Wilson loop operator is given by the following form

$$
W_{R}=\frac{1}{\operatorname{dim} \mathrm{R}} \operatorname{Tr}_{R} \mathrm{P} \exp \left(\oint_{C} \mathrm{~d} \tau\left(i A_{\mu} \dot{x}^{\mu}+\sigma|\dot{x}|\right)\right)
$$

where $R$ is a representation of the gauge group, and $\mathrm{P}$ represents path-ordering, and $C$ denotes a closed world-line parameterized by $x^{\mu}(\tau)$. The supersymmetry variation of this operator is

$$
\delta W_{R} \propto \frac{1}{2}\left(\bar{\epsilon} \gamma_{\mu} \lambda-\bar{\lambda} \gamma_{\mu} \epsilon\right) \dot{x}^{\mu}+\frac{1}{2}(\bar{\epsilon} \lambda-\bar{\lambda} \epsilon)|\dot{x}|
$$

\footnotetext{
${ }^{12} \rho$ are the weights of the representation of the chiral multiplet.
} 
This vanishes for the Killing spinors (2.22) if we take

$$
\dot{x}^{\mu}=\frac{1}{\ell}(1,-1,0)=-v^{\mu} .
$$

Thus, the supersymmetric Wilson loop is parameterized by $\theta=\theta_{1}$ (and $\varphi+\chi=$ const.), and we find that the expectation value for this operator is

$$
\left\langle W_{R}\right\rangle=\frac{1}{\operatorname{dim} \mathrm{R}} \operatorname{Tr}_{R} \exp \left(2 \pi\left(i a_{\varphi}+\ell \sigma_{0}\right)\right) .
$$

\section{Two-dimensional theories}

\subsection{A 2D manifold with a boundary}

We will describe a two dimensional manifold with a boundary on which the supersymmetric field theories constructed. As in the 3D case, we first consider $S^{2}$. The coordinates we will use are $(\theta, \varphi)(0 \leq \theta \leq \pi, 0 \leq \varphi<2 \pi)$ with the metric

$$
\begin{aligned}
\mathrm{d} s^{2} & =\ell^{2}\left(\mathrm{~d} \theta^{2}+\sin ^{2} \theta \mathrm{d} \varphi^{2}\right), \\
\sqrt{g} & =\ell^{2} \sin \theta
\end{aligned}
$$

We choose the following zweibein

$$
e^{1}=\ell \mathrm{d} \theta, \quad e^{2}=\ell \sin \theta \mathrm{d} \varphi
$$

The spin connection is given by

$$
\omega^{a b}=-\varepsilon^{a b} \cos \theta \mathrm{d} \varphi, \quad\left(\varepsilon^{12}=1\right),
$$

and the gamma matrices are

$$
\gamma^{\theta}=\frac{1}{\ell} \gamma^{1}, \quad \gamma^{\varphi}=\frac{1}{\ell \sin \theta} \gamma^{2}
$$

The manifold with the boundary is defined by just restricting the coordinate $\theta$ as

$$
0 \leq \theta \leq \theta_{0}
$$

where $0<\theta_{0} \leq \pi$. Thus the boundary defined by $\theta=\theta_{0}$ is a circle parameterized by $\varphi$, except for $\theta_{0}=\pi$. 


\section{2. $2 \mathrm{D}$ supersymmetric field theories}

Now we will construct the supersymmetric field theories on the 2-dimensional manifold with the boundary.

First, we will summarize the supersymmetry transformations and the supersymmetric invariant Lagrangians of the $\mathcal{N}=(2,2)$ supersymmetric filed theories on the round $S^{2}$ [13, 14].

The (positive) Killing spinors on $S^{2}$ is given by

$$
D_{\mu} \epsilon=\frac{i}{2 \ell} \gamma_{\mu} \epsilon
$$

which is solved with constants $C_{1}, C_{2}$ as

$$
\epsilon=C_{1} e^{i \frac{\varphi}{2}}\left(\begin{array}{c}
i \cos \frac{\theta}{2} \\
-\sin \frac{\theta}{2}
\end{array}\right)+C_{2} e^{-i \frac{\varphi}{2}}\left(\begin{array}{c}
-\sin \frac{\theta}{2} \\
i \cos \frac{\theta}{2},
\end{array}\right)
$$

in our basis.

The supersymmetry transformations of the vector multiplets with the Grassmann odd Killing spinor parameters $\epsilon, \bar{\epsilon}$ are the followings:

$$
\begin{aligned}
\delta A_{\mu}= & -\frac{i}{2}\left(\bar{\epsilon} \gamma_{\mu} \lambda-\bar{\lambda} \gamma_{\mu} \epsilon\right), \quad \delta \sigma_{1}=\frac{1}{2}(\bar{\epsilon} \lambda-\bar{\lambda} \epsilon), \quad \delta \sigma_{2}=-\frac{i}{2}\left(\bar{\epsilon} \gamma_{3} \lambda-\bar{\lambda} \gamma_{3} \epsilon\right), \\
\delta \lambda= & \left(i \gamma_{3} F_{12}-D+i \gamma^{\mu} D_{\mu} \sigma_{1}-\gamma_{3} \gamma^{\mu} D_{\mu} \sigma_{2}-\gamma_{3}\left[\sigma_{1}, \sigma_{2}\right]\right) \epsilon+i \sigma_{1} \gamma^{\mu} D_{\mu} \epsilon-\sigma_{2} \gamma_{3} \gamma^{\mu} D_{\mu} \epsilon, \\
\delta \bar{\lambda}= & \left(i \gamma_{3} F_{12}+D-i \gamma^{\mu} D_{\mu} \sigma_{1}-\gamma_{3} \gamma^{\mu} D_{\mu} \sigma_{2}+\gamma_{3}\left[\sigma_{1}, \sigma_{2}\right]\right) \bar{\epsilon}-i \sigma_{1} \gamma^{\mu} D_{\mu} \bar{\epsilon}-\sigma_{2} \gamma_{3} \gamma^{\mu} D_{\mu} \bar{\epsilon} \\
\delta D= & -\frac{i}{2} \bar{\epsilon}\left(\gamma^{\mu} D_{\mu} \lambda-\left[\lambda, \sigma_{1}\right]+i\left[\gamma_{3} \lambda, \sigma_{2}\right]\right)-\frac{i}{2}\left(D_{\mu} \bar{\lambda} \gamma^{\mu}-\left[\bar{\lambda}, \sigma_{1}\right]+i\left[\bar{\lambda} \gamma_{3}, \sigma_{2}\right]\right) \epsilon \\
& -\frac{i}{2}\left(D_{\mu} \bar{\epsilon} \gamma^{\mu} \lambda+\bar{\lambda} \gamma^{\mu} D_{\mu} \epsilon\right) .
\end{aligned}
$$

For a chiral multiplet of R-charge $q$, the supersymmetry transformations are:

$$
\begin{aligned}
& \delta \phi=\bar{\epsilon} \psi, \quad \delta \psi=\left(i D_{\mu} \phi \gamma^{\mu}+i \sigma_{1} \phi+\gamma_{3} \sigma_{2} \phi\right) \epsilon+\frac{i q}{2} \phi \gamma^{\mu} D_{\mu} \epsilon+\bar{\epsilon} F \\
& \delta \bar{\phi}=\epsilon \bar{\psi}, \quad \delta \bar{\psi}=\left(i D_{\mu} \bar{\phi} \gamma^{\mu}+i \bar{\phi} \sigma_{1}-\gamma_{3} \bar{\phi} \sigma_{2}\right) \bar{\epsilon}+\frac{i q}{2} \bar{\phi} \gamma^{\mu} D_{\mu} \bar{\epsilon}+\epsilon \bar{F} \\
& \delta F=\epsilon\left(i \gamma^{\mu} D_{\mu} \psi-i \sigma_{1} \psi+\gamma_{3} \sigma_{2} \psi-i \lambda \phi\right)+\frac{i q}{2} D_{\mu} \epsilon \gamma^{\mu} \psi \\
& \delta \bar{F}=\bar{\epsilon}\left(i \gamma^{\mu} D_{\mu} \bar{\psi}-i \bar{\psi} \sigma_{1}-\gamma_{3} \bar{\psi} \sigma_{2}+i \bar{\phi} \bar{\lambda}\right)+\frac{i q}{2} D_{\mu} \bar{\epsilon} \gamma^{\mu} \bar{\psi}
\end{aligned}
$$

We can construct several actions which are invariant under the SUSY transformations 
(3.9)-(3.10). The first is the Yang-Mills Lagrangian.13

$$
\begin{aligned}
\frac{1}{g_{\mathrm{YM}}^{2}} \mathcal{L}_{\mathrm{YM}}=\frac{1}{g_{\mathrm{YM}}^{2}} \operatorname{Tr}( & \frac{1}{2}\left(F_{12}-\sigma_{2} / \ell\right)^{2}+\frac{1}{2} D_{\mu} \sigma_{1} D^{\mu} \sigma_{1}+\frac{1}{2} D_{\mu} \sigma_{2} D^{\mu} \sigma_{2}+\frac{1}{2}\left(D+\sigma_{1} / \ell\right)^{2}-\frac{1}{2}\left[\sigma_{1}, \sigma_{2}\right]^{2} \\
+ & \left.\frac{i}{4} \bar{\lambda} \gamma^{\mu} D_{\mu} \lambda+\frac{i}{4} \lambda \gamma^{\mu} D_{\mu} \bar{\lambda}+\frac{i}{2} \bar{\lambda}\left[\sigma_{1}, \lambda\right]+\frac{1}{2} \bar{\lambda} \gamma^{3}\left[\sigma_{2}, \lambda\right]\right)
\end{aligned}
$$

where $g_{\mathrm{YM}}$ is the coupling constant, and $F_{12}$ means $\frac{1}{2} \varepsilon^{a b} F_{a b}$. We can also consider the Fayet Iliopoulos (FI) term:

$$
\mathcal{L}_{\mathrm{FI}}=\operatorname{Tr}\left(-i \zeta D+\frac{i \Theta}{2 \pi} F_{12}\right)
$$

The kinetic terms for a chiral multiplet of R-charge $q$ are

$$
\begin{aligned}
\mathcal{L}_{\text {mat }}= & D_{\mu} \bar{\phi} D^{\mu} \phi+\bar{\phi} \sigma_{1}^{2} \phi+\bar{\phi} \sigma_{2}^{2} \phi+\frac{i q}{\ell} \bar{\phi} \sigma_{1} \phi+\frac{q(2-q)}{4 \ell^{2}} \bar{\phi} \phi+i \bar{\phi} D \phi+\bar{F} F \\
& -\frac{i}{2} \bar{\psi} \gamma^{\mu} D_{\mu} \psi+\frac{i}{2} D_{\mu} \bar{\psi} \gamma^{\mu} \psi+i \bar{\psi} \sigma_{1} \psi-\bar{\psi} \gamma^{3} \sigma_{2} \psi-\frac{q}{2 \ell} \bar{\psi} \psi+i \bar{\psi} \lambda \phi-i \bar{\phi} \bar{\lambda} \psi
\end{aligned}
$$

\subsubsection{The boundary condition}

We study only Dirichlet boundary conditions similar to the 3D theories.

The boundary conditions we will impose for the vector multiplet are

$$
\begin{gathered}
\left.A_{\varphi}\right|_{\theta=\theta_{0}}=a_{\varphi}, \\
\left.\sigma_{1}\right|_{\theta=\theta_{0}}=\sigma_{0}, \\
\left.\sigma_{2}\right|_{\theta=\theta_{0}}=\eta_{0}, \\
-\left.\ell e^{-i \varphi} \gamma^{\theta} \lambda\right|_{\theta=\theta_{0}}=\left.\bar{\lambda}\right|_{\theta=\theta_{0}},
\end{gathered}
$$

where $a_{\varphi}, \sigma_{0}$ and $\eta_{0}$ are constants and commute with each other, and we consider that they are in the Cartan part of the adjoint representation. We do not impose any condition for the other fields ( $A_{\theta}$ and $D$ ). We take the following boundary conditions for chiral multiplets:

$$
\begin{aligned}
& \left.\phi\right|_{\theta=\theta_{0}}=0,\left.\quad e^{i \frac{\theta_{0}}{2} \gamma^{1}} \gamma^{3} e^{-i \frac{\theta_{0}}{2} \gamma^{1}} \psi\right|_{\theta=\theta_{0}}=-\left.\psi\right|_{\theta=\theta_{0}}, \\
& \left.\bar{\phi}\right|_{\theta=\theta_{0}}=0,\left.\quad e^{i \frac{\theta_{0}}{2} \gamma^{1}} \gamma^{3} e^{-i \frac{\theta_{0}}{2} \gamma^{1}} \bar{\psi}\right|_{\theta=\theta_{0}}=\left.\bar{\psi}\right|_{\theta=\theta_{0}} .
\end{aligned}
$$

Under these boundary conditions, the half (or 1/4) of the SUSY is preserved 14 Indeed,

\footnotetext{
${ }^{13}$ Note that the fermion kinetic terms are taken to be symmetrical with respect to $\lambda$ and $\bar{\lambda}$.

14 Only the first equation in (3.19) is needed for the vector multiples and only the last two equations in (3.19) are needed for the chiral multiplets. If there are only vector multiplets or only chiral multiplets, the half of the SUSY preserved, however, if both vector multiplets and chiral multiplets, the $1 / 4$ of the SUSY is preserved.
} 
we can see that the positive Killing spinors which satisfy the relations

$$
\ell e^{-i \varphi} \gamma^{\theta} \epsilon=\bar{\epsilon}, \quad e^{i \frac{\theta}{2} \gamma^{1}} \gamma^{3} e^{-i \frac{\theta}{2} \gamma^{1}} \epsilon=\epsilon, \quad e^{i \frac{\theta}{2} \gamma^{1}} \gamma^{3} e^{-i \frac{\theta}{2} \gamma^{1}} \bar{\epsilon}=-\bar{\epsilon}
$$

generate the supersymmetry transformation which is consistent with the above boundary conditions and under which the actions are invariant (see Appendix B).

The Grassmann even Killing spinors satisfying the relations (3.19) are given by

$$
\epsilon=e^{i \frac{\varphi}{2}}\left(\begin{array}{c}
i \cos \frac{\theta}{2} \\
-\sin \frac{\theta}{2}
\end{array}\right) \quad \text { and } \quad \bar{\epsilon}=e^{-i \frac{\varphi}{2}}\left(\begin{array}{c}
-\sin \frac{\theta}{2} \\
i \cos \frac{\theta}{2}
\end{array}\right)
$$

We can compute the following bi-linears of the spinors:

$$
\begin{aligned}
\bar{\epsilon} \epsilon & =1, \\
\bar{\epsilon} \gamma^{3} \epsilon & =\cos \theta \equiv w, \\
\bar{\epsilon} \gamma^{a} \epsilon & =(0, \sin \theta) \equiv v^{a},
\end{aligned}
$$

which will be used later.

We should check also that the boundary conditions are consistent with the variational principle. The surface terms from variation of the Yang-Mills action are

$$
\frac{1}{g_{\mathrm{YM}}^{2}} \int_{\theta=\theta_{0}} \mathrm{~d} \varphi \sin \theta \operatorname{Tr}\left(\delta A_{\varphi} \frac{F^{\theta \varphi}}{\sin \theta}-\delta A^{\varphi} \sigma_{2}+\delta \sigma_{1} D_{\theta} \sigma_{1}+\delta \sigma_{2} D_{\theta} \sigma_{2}+\frac{i}{4} \bar{\lambda} \gamma_{\theta} \delta \lambda+\frac{i}{4} \lambda \gamma_{\theta} \delta \bar{\lambda}\right) .
$$

which actually vanish for the above boundary conditions. The surface term for the FI term is

$$
\int_{\theta=\theta_{0}} \mathrm{~d} \varphi \frac{i \Theta}{2 \pi} \operatorname{Tr} \delta A_{\varphi}
$$

and the ones for the matter kinetic terms are

$$
\int_{\theta=\theta_{0}} \mathrm{~d} \varphi \sin \theta\left(\delta \bar{\phi} D_{\theta} \phi+D_{\theta} \bar{\phi} \delta \phi-\frac{i}{2} \bar{\psi} \gamma_{\theta} \delta \psi+\frac{i}{2} \delta \bar{\psi} \gamma_{\theta} \psi\right)
$$

We can see that these surface terms vanish with the boundary conditions.

\subsection{Localization}

In this subsection, as in the $3 \mathrm{D}$ theories, we will construct the $\delta$-exact term. 


\subsection{1. $2 \mathrm{D}$ vector multiplet}

For the vector multiplet, we consider the following $\delta$-exact term (ignoring the trace for notational convenience)

$$
\delta V_{\text {vector }}=\frac{1}{4} \delta\left(\left(\delta^{\prime} \lambda\right)^{\dagger} \lambda+\bar{\lambda}\left(\delta^{\prime} \bar{\lambda}\right)^{\dagger}\right)
$$

where

$$
\begin{aligned}
& (\delta \lambda)^{\dagger}=\bar{\epsilon}\left(-i \gamma^{3} F_{12}-D-i \gamma^{\mu} D_{\mu} \sigma_{1}+\gamma^{3} \gamma^{\mu} D_{\mu} \sigma_{2}+\gamma^{3}\left[\sigma_{1}, \sigma_{2}\right]-\frac{1}{\ell} \sigma_{1}+\frac{i}{\ell} \gamma^{3} \sigma_{2}\right), \\
& (\delta \bar{\lambda})^{\dagger}=-\left(i \gamma^{3} F_{12}+D-i \gamma^{\mu} D_{\mu} \sigma_{1}-\gamma^{3} \gamma^{\mu} D_{\mu} \sigma_{2}+\gamma^{3}\left[\sigma_{1}, \sigma_{2}\right]+\frac{1}{\ell} \sigma_{1}-\frac{i}{\ell} \gamma^{3} \sigma_{2}\right) \epsilon .
\end{aligned}
$$

Then, the bosonic part of $\delta$-exact term is computed using

$$
\begin{aligned}
\left(\delta^{\prime} \lambda\right)^{\dagger} \delta \lambda=\bar{\epsilon}^{\prime} \epsilon[ & \left(F_{12}-\sigma_{2} / \ell\right)^{2}+D_{\mu} \sigma_{1} D^{\mu} \sigma_{1}+D_{\mu} \sigma_{2} D^{\mu} \sigma_{2}+\left(D+\sigma_{1} / \ell\right)^{2}-\left[\sigma_{1}, \sigma_{2}\right]^{2} \\
& \left.+2 \varepsilon^{\mu \nu} D_{\mu} \sigma_{1} D_{\nu} \sigma_{2}+2 i F_{12}\left[\sigma_{1}, \sigma_{2}\right]-\frac{2 i}{\ell}\left[\sigma_{1}, \sigma_{2}\right] \sigma_{2}\right] \\
\delta \bar{\lambda}\left(\delta^{\prime} \bar{\lambda}\right)^{\dagger}=\bar{\epsilon}^{\prime} \epsilon[ & \left(F_{12}-\sigma_{2} / \ell\right)^{2}+D_{\mu} \sigma_{1} D^{\mu} \sigma_{1}+D_{\mu} \sigma_{2} D^{\mu} \sigma_{2}+\left(D+\sigma_{1} / \ell\right)^{2}-\left[\sigma_{1}, \sigma_{2}\right]^{2} \\
& \left.-2 \varepsilon^{\mu \nu} D_{\mu} \sigma_{1} D_{\nu} \sigma_{2}-2 i F_{12}\left[\sigma_{1}, \sigma_{2}\right]+\frac{2 i}{\ell}\left[\sigma_{1}, \sigma_{2}\right] \sigma_{2}\right]
\end{aligned}
$$

where we have used the fact that $\bar{\epsilon} \epsilon^{\prime}=-\bar{\epsilon}^{\prime} \epsilon$ by (3.19), as

$$
\begin{aligned}
& \left(\delta^{\prime} \lambda\right)^{\dagger} \delta \lambda+\delta \bar{\lambda}\left(\delta^{\prime} \bar{\lambda}\right)^{\dagger} \\
& =2 \bar{\epsilon}^{\prime} \epsilon\left[\left(F_{12}-\sigma_{2} / \ell\right)^{2}+D_{\mu} \sigma_{1} D^{\mu} \sigma_{1}+D_{\mu} \sigma_{2} D^{\mu} \sigma_{2}+\left(D+\sigma_{1} / \ell\right)^{2}-\left[\sigma_{1}, \sigma_{2}\right]^{2}\right] \\
& =4 \bar{\epsilon}^{\prime} \epsilon \mathcal{L}_{\mathrm{YM}}^{\text {boson }}
\end{aligned}
$$

Next we will compute the fermionic part of the $\delta$-exact term. In

$$
\delta\left(\left(\delta^{\prime} \lambda\right)^{\dagger}\right) \lambda=\bar{\epsilon}^{\prime} \epsilon\left(i \lambda \gamma^{\mu} D_{\mu} \bar{\lambda}-i \lambda\left[\sigma_{1}, \bar{\lambda}\right]+\lambda \gamma^{3}\left[\sigma_{2}, \bar{\lambda}\right]\right)+\left(\bar{\epsilon}^{\prime} \gamma^{\mu} \bar{\epsilon}\right)\left(i\left(D_{\mu} \lambda\right) \lambda\right),
$$

the last term is total derivative and it is equivalent to the surface term:

$$
i\left(\bar{\epsilon}^{\prime} \gamma^{\theta} \bar{\epsilon}\right)(\lambda \lambda)\left|=i \bar{\epsilon}^{\prime} \epsilon\left(\bar{\lambda} \gamma^{\theta} \lambda\right)\right|
$$

where we have used (3.17) and (3.19). Similarly, in

$$
\bar{\lambda} \delta\left(\left(\delta^{\prime} \bar{\lambda}\right)^{\dagger}\right)=\bar{\epsilon}^{\prime} \epsilon\left(i \bar{\lambda} \gamma^{\mu} D_{\mu} \lambda+i \bar{\lambda}\left[\sigma_{1}, \lambda\right]+\bar{\lambda} \gamma^{3}\left[\sigma_{2}, \lambda\right]\right)-\left(\epsilon^{\prime} \gamma^{\mu} \epsilon\right)\left(i\left(D_{\mu} \bar{\lambda}\right) \bar{\lambda}\right)
$$

the last term is also a total derivative and it becomes the surface term:

$$
i\left(\epsilon^{\prime} \gamma^{\theta} \epsilon\right)(\bar{\lambda} \bar{\lambda})\left|=i \bar{\epsilon}^{\prime} \epsilon\left(\bar{\lambda} \gamma^{\theta} \lambda\right)\right|
$$


where we have used (3.17) and (3.19). Thus, the fermionic part of $\delta$-exact term is

$$
\begin{aligned}
\delta\left(\left(\delta^{\prime} \lambda\right)^{\dagger}\right) \lambda+\bar{\lambda} \delta\left(\left(\delta^{\prime} \bar{\lambda}\right)^{\dagger}\right) & =\bar{\epsilon}^{\prime} \epsilon\left(i \bar{\lambda} \gamma^{\mu} D_{\mu} \lambda+i \lambda \gamma^{\mu} D_{\mu} \bar{\lambda}+2 i \bar{\lambda}\left[\sigma_{1}, \lambda\right]+2 \bar{\lambda} \gamma^{3}\left[\sigma_{2}, \lambda\right]\right) \\
& =4 \bar{\epsilon}^{\prime} \epsilon \mathcal{L}_{\mathrm{YM}}^{\text {fermion }}
\end{aligned}
$$

where no surface terms present. Therefore, we find that $\delta V_{\text {vector }}=\mathcal{L}_{Y M}$.

The saddle point of the bosonic part of this $\delta V_{\text {vector }}$ is given by

$$
F_{12}=\frac{\sigma_{2}}{\ell}, D_{\mu} \sigma_{1}=D_{\mu} \sigma_{2}=0, D=-\frac{\sigma_{1}}{\ell},\left[\sigma_{1}, \sigma_{2}\right]=0 .
$$

Using the boundary condition and choosing a gauge condition, the solutions of these equations are

$$
A=\ell \eta_{0}(\kappa-\cos \theta) \mathrm{d} \varphi=a_{\varphi} \mathrm{d} \varphi, \quad \sigma_{1}=-\ell D=\sigma_{0}, \quad \sigma_{2}=\eta_{0}
$$

where $\kappa=1(\kappa=-1)$ for the patch covering $S^{2}$ except a point $\theta=\pi(\theta=0) 15$ In the case for $S^{2}$ [13, 14], since the flux $2 \ell^{2} F_{12}$ is GNO quantized [53], $\rho\left(2 \ell \eta_{0}\right)$ should be an integer for any representation $R$ of the gauge group $G$ and any weight $\rho \in R$. For $\theta_{0}<\pi$, however, the flux does not need to be quantized.

\subsubsection{D chiral multiplet}

For the chiral multiplet, we consider the following $\delta$-exact term

$$
\delta V_{\text {chiral }}=\frac{1}{2} \delta\left[\left(\delta^{\prime} \psi\right)^{\dagger} \psi+\bar{\psi}\left(\delta^{\prime} \bar{\psi}\right)^{\dagger}\right]+\frac{q-1}{2 \ell} \delta\left[\bar{\phi} \delta^{\prime} \phi-\left(\delta^{\prime} \bar{\phi}\right) \phi\right]
$$

where

$$
\begin{aligned}
\left(\delta^{\prime} \psi\right)^{\dagger} & \equiv \bar{\epsilon}^{\prime}\left(-i D_{\mu} \bar{\phi} \gamma^{\mu}-i \bar{\phi} \sigma_{1}+\bar{\phi} \sigma_{2} \gamma^{3}-\frac{q}{2 \ell} \bar{\phi}\right)-\epsilon^{\prime} \bar{F} \\
\left(\delta^{\prime} \bar{\psi}\right)^{\dagger} & \equiv-\left(i D_{\mu} \phi \gamma^{\mu}-i \sigma_{1} \phi+\sigma_{2} \phi \gamma^{3}-\frac{q}{2 \ell} \phi\right) \epsilon^{\prime}+\bar{\epsilon}^{\prime} F
\end{aligned}
$$

The bosonic part of $\delta V_{\text {chiral }}$ is given by

$$
\begin{aligned}
& \frac{1}{2}\left(\delta^{\prime} \psi\right)^{\dagger} \delta \psi+\delta \bar{\psi}\left(\delta^{\prime} \bar{\psi}\right)^{\dagger}+\frac{q-1}{2 \ell}\left[\bar{\phi}\left(\delta \delta^{\prime} \phi\right)-\left(\delta \delta^{\prime} \bar{\phi}\right) \phi\right] \\
& =\bar{\epsilon}^{\prime} \epsilon\left(D_{\mu} \bar{\phi} D^{\mu} \phi+\bar{\phi} \sigma_{1}^{2} \phi+\bar{\phi} \sigma_{2}^{2} \phi+i \frac{q-1}{\ell} \bar{\phi} \sigma_{1} \phi-\frac{q(q-2)}{4 \ell^{2}} \bar{\phi} \phi+\bar{F} F\right) \\
& \quad+i \bar{\epsilon}^{\prime} \gamma^{\mu} \epsilon\left[\frac{1}{2 \ell}\left(D_{\mu} \bar{\phi} \phi-\bar{\phi} D_{\mu} \phi\right)-i \varepsilon_{\mu \nu}\left(D^{\nu} \bar{\phi} \sigma_{2} \phi+\bar{\phi} \sigma_{2} D^{\nu} \phi\right)\right] \\
& \quad+i \bar{\epsilon}^{\prime} \gamma^{3} \epsilon\left(i \varepsilon^{\mu \nu} D_{\mu} \bar{\phi} D_{\nu} \phi+\frac{i}{\ell} \bar{\phi} \sigma_{2} \phi\right) .
\end{aligned}
$$

\footnotetext{
${ }^{15}$ We can take $\kappa=1$ for the case where $\theta_{0}<\pi$.
} 
Similarly, the fermionic part can be computed as

$$
\begin{aligned}
& \frac{1}{2} \delta\left(\left(\delta^{\prime} \psi\right)^{\dagger}\right) \psi+\bar{\psi} \delta\left(\left(\delta^{\prime} \bar{\psi}\right)^{\dagger}\right)+\frac{q-1}{2 \ell}\left[\delta \bar{\phi} \delta^{\prime} \phi-\delta^{\prime} \bar{\phi} \delta \phi\right] \\
& =\bar{\epsilon}^{\prime} \epsilon\left(\frac{i}{2} D_{\mu} \bar{\psi} \gamma^{\mu} \psi-\frac{i}{2} \bar{\psi} \gamma^{\mu} D_{\mu} \psi+i \bar{\psi} \sigma_{1} \psi-\bar{\psi} \sigma_{2} \gamma^{3} \psi-\frac{3 i}{4} \bar{\phi} \bar{\lambda} \psi+\frac{3 i}{4} \bar{\psi} \lambda \phi-\frac{q}{2 \ell} \bar{\psi} \psi\right) \\
& \quad+i \bar{\epsilon}^{\prime} \gamma^{\mu} \epsilon\left[\frac{i}{2} \varepsilon_{\mu \nu}\left(D^{\nu} \bar{\psi} \gamma^{3} \psi+\bar{\psi} \gamma^{3} D^{\nu} \psi\right)+\frac{1}{4} \bar{\phi} \bar{\lambda} \gamma_{\mu} \psi-\frac{1}{4} \bar{\psi} \gamma_{\mu} \lambda \phi-\frac{i}{2 \ell} \bar{\psi} \gamma_{\mu} \psi\right] \\
& \quad+i \bar{\epsilon}^{\prime} \gamma^{3} \epsilon\left(\frac{1}{2} D_{\mu} \bar{\psi} \gamma_{3} \gamma^{\mu} \psi+\frac{1}{2} \bar{\psi} \gamma_{3} \gamma^{\mu} D_{\mu} \psi+\frac{1}{4} \bar{\phi} \bar{\lambda} \gamma_{3} \psi-\frac{1}{4} \bar{\psi} \gamma_{3} \lambda \phi-\frac{i}{\ell} \bar{\psi} \gamma_{3} \psi\right) \\
& \quad-\frac{i}{4} \epsilon^{\prime} \gamma^{\mu} \epsilon \bar{\psi} \gamma_{\mu} \bar{\lambda} \phi+\frac{i}{4} \bar{\epsilon}^{\prime} \gamma^{\mu} \bar{\epsilon} \bar{\phi} \lambda \gamma_{\mu} \psi-\frac{i}{4} \epsilon^{\prime} \gamma^{3} \epsilon \bar{\psi} \gamma_{3} \bar{\lambda} \phi+\frac{i}{4} \bar{\epsilon}^{\prime} \gamma^{3} \bar{\epsilon} \bar{\phi} \lambda \gamma_{3} \psi
\end{aligned}
$$

Therefore,

$$
\begin{gathered}
\delta V_{\text {chiral }}=\bar{\epsilon}^{\prime} \epsilon\left(D_{\mu} \bar{\phi} D^{\mu} \phi+\bar{\phi} \sigma_{1}^{2} \phi+\bar{\phi} \sigma_{2}^{2} \phi+i \frac{q-1}{\ell} \bar{\phi} \sigma_{1} \phi-\frac{q(q-2)}{4 \ell^{2}} \bar{\phi} \phi+\bar{F} F\right. \\
\left.+\frac{i}{2} D_{\mu} \bar{\psi} \gamma^{\mu} \psi-\frac{i}{2} \bar{\psi} \gamma^{\mu} D_{\mu} \psi+i \bar{\psi} \sigma_{1} \psi-\bar{\psi} \sigma_{2} \gamma^{3} \psi-\frac{3 i}{4} \bar{\phi} \bar{\lambda} \psi+\frac{3 i}{4} \bar{\psi} \lambda \phi-\frac{q}{2 \ell} \bar{\psi} \psi\right) \\
+i \bar{\epsilon}^{\prime} \gamma^{\mu} \epsilon\left[\frac{1}{2 \ell}\left(D_{\mu} \bar{\phi} \phi-\bar{\phi} D_{\mu} \phi\right)-i \varepsilon_{\mu \nu}\left(D^{\nu} \bar{\phi} \sigma_{2} \phi+\bar{\phi} \sigma_{2} D^{\nu} \phi\right)\right. \\
\left.+\frac{i}{2} \varepsilon_{\mu \nu}\left(D^{\nu} \bar{\psi} \gamma^{3} \psi+\bar{\psi} \gamma^{3} D^{\nu} \psi\right)+\frac{1}{4} \bar{\phi} \bar{\lambda} \gamma_{\mu} \psi-\frac{1}{4} \bar{\psi} \gamma_{\mu} \lambda \phi-\frac{i}{2 \ell} \bar{\psi} \gamma_{\mu} \psi\right] \\
+i \bar{\epsilon}^{\prime} \gamma^{3} \epsilon\left(i \varepsilon^{\mu \nu} D_{\mu} \bar{\phi} D_{\nu} \phi+\frac{i}{\ell} \bar{\phi} \sigma_{2} \phi\right. \\
\left.+\frac{1}{2} D_{\mu} \bar{\psi} \gamma_{3} \gamma^{\mu} \psi+\frac{1}{2} \bar{\psi} \gamma_{3} \gamma^{\mu} D_{\mu} \psi+\frac{1}{4} \bar{\phi} \bar{\lambda} \gamma_{3} \psi-\frac{1}{4} \bar{\psi} \gamma_{3} \lambda \phi-\frac{i}{\ell} \bar{\psi} \gamma_{3} \psi\right) \\
-\frac{i}{4} \epsilon^{\prime} \gamma^{\mu} \epsilon \bar{\psi} \gamma_{\mu} \bar{\lambda} \phi+\frac{i}{4} \bar{\epsilon}^{\prime} \gamma^{\mu} \bar{\epsilon} \bar{\phi} \lambda \gamma_{\mu} \psi-\frac{i}{4} \epsilon^{\prime} \gamma^{3} \epsilon \bar{\psi} \gamma_{3} \bar{\lambda} \phi+\frac{i}{4} \bar{\epsilon}^{\prime} \gamma^{3} \bar{\epsilon} \bar{\phi} \lambda \gamma_{3} \psi .
\end{gathered}
$$

If we use the $\delta$-exact term $\delta\left[\left(\delta^{\prime} \psi\right)^{\dagger} \psi+\bar{\psi}\left(\delta^{\prime} \bar{\psi}\right)^{\dagger}\right] / 2$, which is manifestly positive definite, instead of $\delta V_{\text {chiral }}$, the saddle point is given by $\phi=F=\bar{\phi}=\bar{F}=0$. Because the addition of $\delta\left[\bar{\phi} \delta^{\prime} \phi-\left(\delta^{\prime} \bar{\phi}\right) \phi\right](q-1) /(2 \ell)$ will not change the 1-loop determinant, we will use $\delta V_{\text {chiral }}$ for simplicity of later computation.

\subsection{One-loop determinant}

In this subsection, we will compute the 1-loop determinant for the $\delta$-exact action in the similar way as in subsection 2.4. The computation will be basically follow [15].

\subsubsection{Vector multiplet}

We will compute the 1-loop determinant for the vector multiplet. 
We expand the fields around the saddle point as

$$
\begin{aligned}
A_{\mu} & =a_{\mu}+\frac{1}{\sqrt{t}} \hat{A}_{\mu}, \quad \sigma_{1}=\sigma_{0}+\frac{1}{\sqrt{t}} \hat{\sigma}_{1}, \\
\sigma_{2} & =\eta_{0}+\frac{1}{\sqrt{t}} \hat{\sigma}_{2}, \quad D=-\frac{\sigma_{0}}{\ell}+\frac{1}{\sqrt{t}} \hat{D} \\
\lambda & =\frac{1}{\sqrt{t}} \hat{\lambda}, \quad \bar{\lambda}=\frac{1}{\sqrt{t}} \hat{\bar{\lambda}},
\end{aligned}
$$

where

$$
a_{\theta}=0, \quad a_{\varphi}=\ell \eta_{0}(\kappa-\cos \theta) .
$$

The boundary conditions for the fluctuation fields at $\theta=\theta_{0}$ are

$$
\hat{A}_{\varphi}\left|=\hat{\sigma}_{1}\right|=\hat{\sigma}_{2} \mid=0 \text {. }
$$

Then, performing the integral by parts, we have

$$
\begin{aligned}
t \int \mathrm{d}^{2} x \sqrt{g} \mathcal{L}_{\mathrm{YM}}=\int \mathrm{d}^{2} x \sqrt{g} \operatorname{Tr} & -\frac{1}{2} A^{\mu}\left(* D^{(a)} * D^{(a)} A\right)_{\mu}-\varepsilon^{\mu \nu} \frac{\sigma_{2}}{\ell} D_{\mu}^{(a)} A_{\nu} \\
& +\frac{1}{2}\left[\sigma_{0}, A^{\mu}\right]\left[\sigma_{0}, A_{\mu}\right]+\frac{1}{2}\left[\eta_{0}, A^{\mu}\right]\left[\eta_{0}, A_{\mu}\right] \\
& -\frac{1}{2} \sigma_{1}\left(* D^{(a)} * D^{(a)} \sigma_{1}\right)+i\left[\sigma_{0}, A^{\mu}\right] D_{\mu}^{(a)} \sigma_{1} \\
& -\frac{1}{2} \sigma_{2}\left(* D^{(a)} * D^{(a)} \sigma_{2}\right)+i\left[\eta_{0}, A^{\mu}\right] D_{\mu}^{(a)} \sigma_{2}+\frac{\sigma_{2}^{2}}{2 \ell^{2}} \\
& +\frac{1}{2}\left(D+\sigma_{1} / \ell\right)^{2}-\frac{1}{2}\left(\left[\sigma_{0}, \sigma_{2}\right]-\left[\eta_{0}, \sigma_{1}\right]\right)^{2} \\
& \left.+\frac{i}{4} \bar{\lambda} \gamma^{\mu} D_{\mu}^{(a)} \lambda+\frac{i}{4} \lambda \gamma^{\mu} D_{\mu}^{(a)} \bar{\lambda}+\frac{i}{2} \bar{\lambda}\left[\sigma_{0}, \lambda\right]+\frac{1}{2} \bar{\lambda} \gamma^{3}\left[\eta_{0}, \lambda\right]\right) \\
+ & \mathcal{O}\left(t^{-1 / 2}\right),
\end{aligned}
$$

where the covariant derivative $D^{(a)}$ means $D_{\mu}^{(a)}=\nabla_{\mu}-i\left[a_{\mu}, \cdot\right]$ and we have omitted the hat symbols for the fluctuation fields.

As explained in [15], for evaluating the 1-loop factor for bosonic fields, only the eigenvalues of the bosonic eigenmodes which are orthogonal to the "non-physical modes" should be included. Using the results in [15], we can easily see that the orthogonal conditions are given by

$$
* D^{(a)} * A=i\left[\eta_{0}, \sigma_{2}\right], \quad \sigma_{1}=0
$$

for our conventions. The latter condition, $\sigma_{1}=0$, implies that we need to consider only $A_{\mu}$ and $\sigma_{2} .16$

\footnotetext{
${ }^{16}$ The eigenvalue problem for the bosonic kinetic operator in (3.51) is also consistent with $\sigma_{1}=0$ if the former condition, $* D^{(a)} * A=i\left[\eta_{0}, \sigma_{2}\right]$, is imposed.
} 
Using the Cartan decomposition, all the adjoint fields $X$ can be decomposed as

$$
X=\sum_{i} X^{i} H_{i}+\sum_{\alpha \in \Delta_{+}}\left(X^{\alpha} E_{\alpha}+X^{-\alpha} E_{-\alpha}\right)
$$

where $H_{i}$ are the Cartan generators and $\Delta_{+}$is the set of the positive roots. The generators $E_{\alpha}$ are normalized as $\operatorname{Tr}\left(E_{\alpha} E_{\beta}\right)=\delta_{\alpha+\beta}$. Then, the quadratic terms we consider have the following formit 17

$$
\left(A^{-\alpha}, \sigma_{2}^{-\alpha}\right) \Delta_{b}\left(\begin{array}{c}
A^{\alpha} \\
\sigma_{2}^{\alpha}
\end{array}\right)+\frac{1}{2}\left(\lambda^{-\alpha}, \bar{\lambda}^{-\alpha}\right)\left(\begin{array}{cc}
0 & \Delta_{\bar{\lambda}} \\
\Delta_{\lambda} & 0
\end{array}\right)\left(\begin{array}{l}
\lambda^{\alpha} \\
\bar{\lambda}^{\alpha}
\end{array}\right),
$$

where

$$
\begin{aligned}
& \Delta_{b}=\left(\begin{array}{cc}
-* D^{(a)} * D^{(a)}+\alpha\left(\sigma_{0}\right)^{2}+\alpha\left(\eta_{0}\right)^{2} & -i \alpha\left(\eta_{0}\right) D^{(a)}+\frac{1}{\ell} * D^{(a)} \\
-i \alpha\left(\eta_{0}\right) * D^{(a)} *-\frac{1}{\ell} * D^{(a)} & -* D^{(a)} * D^{(a)}+\alpha\left(\sigma_{0}\right)^{2}+\frac{1}{\ell^{2}}
\end{array}\right) \\
& \Delta_{\lambda}=i \gamma^{\mu} D_{\mu}^{(a)}+i \alpha\left(\sigma_{0}\right)+\gamma^{3} \alpha\left(\eta_{0}\right), \quad \Delta_{\bar{\lambda}}=i \gamma^{\mu} D_{\mu}^{(a)}-i \alpha\left(\sigma_{0}\right)+\gamma^{3} \alpha\left(\eta_{0}\right) .
\end{aligned}
$$

Accordingly, we will consider the eigenvalue problem for the above kinetic terms as in subsection 2.4.

First, we will construct a correspondence between the bosonic eigenmodes and fermionic eigenmodes as in subsection 2.4 and as in [15]. Let $\left(A^{\alpha}, \sigma_{2}^{\alpha}\right)$ be an eigenmode for $\Delta_{b}$ :

$$
\Delta_{b}\left(\begin{array}{c}
A^{\alpha} \\
\sigma_{2}^{\alpha}
\end{array}\right)=M^{2}\left(\begin{array}{c}
A^{\alpha} \\
\sigma_{2}^{\alpha}
\end{array}\right), \quad * D^{(a)} * A^{\alpha}=i \alpha\left(\eta_{0}\right) \sigma_{2}^{\alpha} .
$$

Then, if we define

$$
\begin{aligned}
& \lambda_{1}^{\alpha} \equiv\left(\gamma^{\mu} A_{\mu}^{\alpha}+\gamma^{3} \sigma_{2}^{\alpha}\right) \epsilon \\
& \lambda_{2}^{\alpha} \equiv-\left(* D^{(a)} A^{\alpha}\right) \gamma^{3} \epsilon+\left(* D^{(a)} \sigma_{2}^{\alpha}\right)_{\mu} \gamma^{\mu} \epsilon+\frac{1}{\ell} \gamma^{3} \sigma_{2}^{\alpha} \epsilon+\alpha\left(\eta_{0}\right) A_{\mu}^{\alpha} \gamma^{3} \gamma^{\mu} \epsilon, \\
& \bar{\lambda}_{1}^{\alpha} \equiv-\left(\gamma^{\mu} A_{\mu}^{\alpha}+\gamma^{3} \sigma_{2}^{\alpha}\right) \bar{\epsilon} \\
& \bar{\lambda}_{2}^{\alpha} \equiv-\left(* D^{(a)} A^{\alpha}\right) \gamma^{3} \bar{\epsilon}+\left(* D^{(a)} \sigma_{2}^{\alpha}\right)_{\mu} \gamma^{\mu} \bar{\epsilon}+\frac{1}{\ell} \gamma^{3} \sigma_{2}^{\alpha} \bar{\epsilon}+\alpha\left(\eta_{0}\right) A_{\mu}^{\alpha} \gamma^{3} \gamma^{\mu} \bar{\epsilon}
\end{aligned}
$$

we can obtain fermionic eigenmodes for $\Delta_{\lambda}$ and $\Delta_{\bar{\lambda}}$ from the bosonic eigenmode,

$$
\Delta_{\lambda} \lambda_{ \pm}^{\alpha}=\nu_{ \pm} \lambda_{ \pm}^{\alpha}, \quad \Delta_{\bar{\lambda}} \bar{\lambda}_{ \pm}^{\alpha}=-\nu_{ \pm} \bar{\lambda}_{ \pm}^{\alpha},
$$

where

$$
\begin{aligned}
& \lambda_{ \pm}^{\alpha} \equiv \pm \sqrt{M^{2}-\alpha\left(\sigma_{0}\right)^{2}} \lambda_{1}^{\alpha}+\lambda_{2}^{\alpha}, \quad \bar{\lambda}_{ \pm}^{\alpha} \equiv \pm \sqrt{M^{2}-\alpha\left(\sigma_{0}\right)^{2}} \bar{\lambda}_{1}^{\alpha}+\bar{\lambda}_{2}^{\alpha}, \\
& \nu_{ \pm}=i \alpha\left(\sigma_{0}\right) \pm \sqrt{M^{2}-\alpha\left(\sigma_{0}\right)^{2}} \text {. }
\end{aligned}
$$

\footnotetext{
17 The contributions from the Cartan part $X^{i}$ are the form $\ell^{p}$, thus, we have neglected them.
} 
One can show that these fermionic eigenmodes satisfy the boundary condition

$$
-\ell e^{-i \varphi} \gamma^{\theta} \lambda_{ \pm}^{\alpha}\left|=\bar{\lambda}_{ \pm}^{\alpha}\right|
$$

since $\left(A, \sigma_{2}\right)$ satisfies the boundary condition (3.50). On the other hand, if $\lambda$ and $\bar{\lambda}$ are fermionic eigenmodes,

$$
\Delta_{\lambda} \lambda^{\alpha}=\nu \lambda^{\alpha}, \quad \Delta_{\bar{\lambda}} \bar{\lambda}^{\alpha}=-\nu \bar{\lambda}^{\alpha}
$$

which satisfy the boundary condition

$$
-\ell e^{-i \varphi} \gamma^{\theta} \lambda^{\alpha}\left|=\bar{\lambda}^{\alpha}\right|
$$

then we can obtain an eigenmode for $\Delta_{b}$,

$$
\begin{aligned}
A_{\mu}^{\alpha} & \equiv\left(\nu-i \alpha\left(\sigma_{0}\right)\right)\left(\bar{\epsilon} \gamma_{\mu} \lambda^{\alpha}+\epsilon \gamma_{\mu} \bar{\lambda}^{\alpha}\right)-i D_{\mu}^{(a)}\left(\bar{\epsilon} \lambda^{\alpha}-\epsilon \bar{\lambda}^{\alpha}\right) \\
\sigma_{2}^{\alpha} & \equiv\left(\nu-i \alpha\left(\sigma_{0}\right)\right)\left(\bar{\epsilon} \gamma_{3} \lambda^{\alpha}+\epsilon \gamma_{3} \bar{\lambda}^{\alpha}\right)-\alpha\left(\eta_{0}\right)\left(\bar{\epsilon} \lambda^{\alpha}-\epsilon \bar{\lambda}^{\alpha}\right) .
\end{aligned}
$$

This mode satisfies the boundary conditions (3.50) and the condition

$$
* D^{(a)} * A^{\alpha}=i \alpha\left(\eta_{0}\right) \sigma_{2}^{\alpha}
$$

The corresponding eigenvalue is $\left(\nu-i \alpha\left(\sigma_{0}\right)\right)^{2}+\alpha\left(\sigma_{0}\right)^{2}$

$$
\Delta_{b}\left(\begin{array}{c}
A^{\alpha} \\
\sigma_{2}^{\alpha}
\end{array}\right)=\left[\left(\nu-i \alpha\left(\sigma_{0}\right)\right)^{2}+\alpha\left(\sigma_{0}\right)^{2}\right]\left(\begin{array}{c}
A^{\alpha} \\
\sigma_{2}^{\alpha}
\end{array}\right) .
$$

Due to the correspondence between the bosonic eigenmodes and fermionic eigenmodes, the eigenvalues for bosonic modes are almost canceled by the ones for fermionic eigenmodes in the evaluation for 1-loop determinant. Therefore, we will consider only the eigenmodes which are not canceled.

\section{Unpaired eigenmodes}

We consider unpaired eigenmodes whose eigenvalues are not canceled. In Appendix A, we summarize some useful formulas for Killing spinors (3.20) and their bi-linears, which will be used in the following calculation.

We consider unpaired bosonic eigenmodes $\left(A_{\mu}, \sigma_{2}\right)$,

$$
\Delta_{b}\left(\begin{array}{c}
A^{\alpha} \\
\sigma_{2}^{\alpha}
\end{array}\right)=M^{2}\left(\begin{array}{c}
A^{\alpha} \\
\sigma_{2}^{\alpha}
\end{array}\right)
$$


which satisfy $* D^{(a)} * A^{\alpha}=i \alpha\left(\eta_{0}\right) \sigma_{2}^{\alpha}$ and

$$
C \lambda_{1}+\lambda_{2}=0, \quad C \bar{\lambda}_{1}+\bar{\lambda}_{2}=0
$$

where $C=\sqrt{M^{2}-\alpha\left(\sigma_{0}\right)^{2}}$ or $C=-\sqrt{M^{2}-\alpha\left(\sigma_{0}\right)^{2}}$ and $\lambda_{1}, \lambda_{2}, \bar{\lambda}_{1}, \bar{\lambda}_{2}$ are given by (3.58)(3.61). Accordingly, $\left(A_{\mu}, \sigma_{2}\right)$ should satisfy the following equations:

$$
\begin{aligned}
& C\left(v^{\mu} A_{\mu}+w \sigma_{2}\right)=0, \quad i C \tilde{v}^{\mu} A_{\mu}-\left(* D^{(a)} A\right)+\frac{1}{\ell} \sigma_{2}=0 . \\
& C \sigma_{2}+i \tilde{v}^{\mu}\left(* D^{(a)} \sigma_{2}\right)_{\mu}+\alpha\left(\eta_{0}\right) v^{\mu} A_{\mu}=0, \\
& w\left(* D^{(a)} A\right)-v^{\mu}\left(* D^{(a)} \sigma_{2}\right)_{\mu}-\frac{w}{\ell} \sigma_{2}-i \alpha\left(\eta_{0}\right) \tilde{v}^{\mu} A_{\mu}=0
\end{aligned}
$$

where the definitions of $w, v^{\mu}, \tilde{v}^{\mu}$ are given by (A.20). If we make the ansatz

$$
A=f_{1}(\theta) e^{i m \varphi} e^{1}+f_{2}(\theta) e^{i m \varphi} e^{2}, \quad \sigma_{2}=f(\theta) e^{i m \varphi}
$$

where $m$ is an integer, then we can find that

$$
\ell C=-m+\kappa \ell \alpha\left(\eta_{0}\right)
$$

so that the condition (3.73) should be consistent with the eigenmode equation (3.72). The remaining conditions imply that $f_{1}, f_{2}$ and $f$ should satisfy the following equations

$$
\begin{aligned}
\sin \theta f_{2}+\cos \theta f & =0, \\
\partial_{\theta}\left(\sin \theta f_{1}\right) & =-i\left(\ell \alpha\left(\eta_{0}\right) \cos \theta-\ell C\right) f_{2}-i \ell \alpha\left(\eta_{0}\right) \frac{\sin ^{2} \theta}{\cos \theta} f_{2}, \\
\partial_{\theta}\left(\sin \theta f_{2}\right) & =i\left(\ell \alpha\left(\eta_{0}\right) \cos \theta-\ell C \cos ^{2} \theta\right) f_{1}-\frac{\sin ^{2} \theta}{\cos \theta} f_{2} .
\end{aligned}
$$

One can find that general solutions of these equations are given by

$$
\begin{aligned}
f_{1}(\theta)= & -i C_{1}\left(\sin \frac{\theta}{2}\right)^{-(\kappa-1) \ell \alpha\left(\eta_{0}\right)}\left(\cos \frac{\theta}{2}\right)^{-(\kappa+1) \ell \alpha\left(\eta_{0}\right)} \sin ^{m-1} \theta \\
& +i C_{2}\left(\sin \frac{\theta}{2}\right)^{(\kappa-1) \ell \alpha\left(\eta_{0}\right)}\left(\cos \frac{\theta}{2}\right)^{(\kappa+1) \ell \alpha\left(\eta_{0}\right)} \sin ^{-m-1} \theta \\
f_{2}(\theta)= & C_{1} \cos \theta\left(\sin \frac{\theta}{2}\right)^{-(\kappa-1) \ell \alpha\left(\eta_{0}\right)}\left(\cos \frac{\theta}{2}\right)^{-(\kappa+1) \ell \alpha\left(\eta_{0}\right)} \sin ^{m-1} \theta \\
& +C_{2} \cos \theta\left(\sin \frac{\theta}{2}\right)^{(\kappa-1) \ell \alpha\left(\eta_{0}\right)}\left(\cos \frac{\theta}{2}\right)^{(\kappa+1) \ell \alpha\left(\eta_{0}\right)} \sin ^{-m-1} \theta \\
f(\theta)= & -C_{1}\left(\sin \frac{\theta}{2}\right)^{-(\kappa-1) \ell \alpha\left(\eta_{0}\right)}\left(\cos \frac{\theta}{2}\right)^{-(\kappa+1) \ell \alpha\left(\eta_{0}\right)} \sin ^{m} \theta \\
& -C_{2}\left(\sin \frac{\theta}{2}\right)^{(\kappa-1) \ell \alpha\left(\eta_{0}\right)}\left(\cos \frac{\theta}{2}\right)^{(\kappa+1) \ell \alpha\left(\eta_{0}\right)} \sin ^{-m} \theta
\end{aligned}
$$


where $C_{1}$ and $C_{2}$ are constants. The regularity at $\theta=0$ requires $C_{1}=0$ or $C_{2}=0$. Thus, in the case where $\theta_{0}<\pi$, there is no nontrivial solution which satisfies the boundary condition. For the special case that $\theta_{0}=\pi$, there are solutions:

$$
\begin{aligned}
& C_{2}=0, \\
& \ell C=-m+\ell \alpha\left(\eta_{0}\right), \quad \begin{cases}m \geq 2 \ell \alpha\left(\eta_{0}\right)+2 & \text { for } \ell \alpha\left(\eta_{0}\right) \geq 0 \\
m \geq 1 & \text { for } \quad \ell \alpha\left(\eta_{0}\right)<0,\end{cases}
\end{aligned}
$$

or

$$
\begin{aligned}
& C_{1}=0, \\
& \ell C=-m+\ell \alpha\left(\eta_{0}\right), \quad \begin{cases}m \leq-1 & \text { for } \ell \alpha\left(\eta_{0}\right)>0 \\
m \leq 2 \ell \alpha\left(\eta_{0}\right)-2 & \text { for } \quad \ell \alpha\left(\eta_{0}\right) \leq 0,\end{cases}
\end{aligned}
$$

where we assume that $2 \ell \alpha\left(\eta_{0}\right)$ takes an integer value. For $S^{2}$, there are solutions:

$$
\begin{aligned}
& C_{2}=0, \\
& \ell C=-m+\ell \alpha\left(\eta_{0}\right), \quad \begin{cases}m \geq 2 \ell \alpha\left(\eta_{0}\right)+1 & \text { for } \ell \alpha\left(\eta_{0}\right) \geq 0 \\
m \geq 1 & \text { for } \quad \ell \alpha\left(\eta_{0}\right)<0,\end{cases}
\end{aligned}
$$

or

$$
\begin{aligned}
& C_{1}=0, \\
& \ell C=-m+\ell \alpha\left(\eta_{0}\right), \quad \begin{cases}m \leq-1 & \text { for } \ell \alpha\left(\eta_{0}\right)>0 \\
m \leq 2 \ell \alpha\left(\eta_{0}\right)-1 & \text { for } \ell \alpha\left(\eta_{0}\right) \leq 0 .\end{cases}
\end{aligned}
$$

Thus, shifting $m$ appropriately, $\ell C$ is given by

$$
\ell C= \begin{cases}-m-\left|\ell \alpha\left(\eta_{0}\right)\right| & (m \geq 1) \\ -m+\left|\ell \alpha\left(\eta_{0}\right)\right| & (m \leq-1) .\end{cases}
$$

Next, we consider unpaired fermionic eigenmodes,

$$
\Delta_{\lambda} \lambda^{\alpha}=\nu \lambda^{\alpha}, \quad \Delta_{\bar{\lambda}} \bar{\lambda}^{\alpha}=-\nu \bar{\lambda}^{\alpha},
$$

which satisfy

$$
\begin{aligned}
& \left(\nu-i \alpha\left(\sigma_{0}\right)\right)\left(\bar{\epsilon} \gamma_{\mu} \lambda^{\alpha}+\epsilon \gamma_{\mu} \bar{\lambda}^{\alpha}\right)-i D_{\mu}^{(a)}\left(\bar{\epsilon} \lambda^{\alpha}-\epsilon \bar{\lambda}^{\alpha}\right)=0 \\
& \left(\nu-i \alpha\left(\sigma_{0}\right)\right)\left(\bar{\epsilon} \gamma_{3} \lambda^{\alpha}+\epsilon \gamma_{3} \bar{\lambda}^{\alpha}\right)-\alpha\left(\eta_{0}\right)\left(\bar{\epsilon} \lambda^{\alpha}-\epsilon \bar{\lambda}^{\alpha}\right)=0
\end{aligned}
$$


We can expand $\lambda$ and $\bar{\lambda}$ as

$$
\lambda^{\alpha}=\Lambda \bar{\epsilon}+\Lambda^{\prime} \gamma^{3} \bar{\epsilon}, \quad \bar{\lambda}^{\alpha}=\bar{\Lambda} \epsilon+\bar{\Lambda}^{\prime} \gamma^{3} \epsilon
$$

where $\Lambda, \Lambda^{\prime}, \bar{\Lambda}$ and $\bar{\Lambda}^{\prime}$ are scalars. Then the boundary conditions can be written as

$$
e^{-i \varphi} \Lambda\left|+e^{i \varphi} \bar{\Lambda}\right|=0, \quad e^{-i \varphi} \Lambda^{\prime}\left|-e^{i \varphi} \bar{\Lambda}^{\prime}\right|=0 .
$$

The eigenvalue equation $\Delta_{\lambda} \lambda^{\alpha}=\nu \lambda^{\alpha}$ is equivalent to

$$
\begin{aligned}
& i v^{\mu} D_{\mu}^{(a)} \Lambda+\tilde{v}^{\mu} D_{\mu}^{(a)} \Lambda^{\prime}+\frac{1}{\ell}\left(\Lambda+w \Lambda^{\prime}\right)+\alpha\left(\eta_{0}\right)\left(w \Lambda-\Lambda^{\prime}\right)=-\left(\nu-i \alpha\left(\sigma_{0}\right)\right)\left(\Lambda-w \Lambda^{\prime}\right), \\
& \tilde{v}^{\mu} D_{\mu}^{(a)} \Lambda+i v^{\mu} D_{\mu}^{(a)} \Lambda^{\prime}+\frac{1}{\ell}\left(w \Lambda+\Lambda^{\prime}\right)+\alpha\left(\eta_{0}\right)\left(\Lambda-w \Lambda^{\prime}\right)=-\left(\nu-i \alpha\left(\sigma_{0}\right)\right)\left(w \Lambda-\Lambda^{\prime}\right),
\end{aligned}
$$

and $\Delta_{\bar{\lambda}} \bar{\lambda}^{\alpha}=-\nu \bar{\lambda}^{\alpha}$ is equivalent to

$$
\begin{aligned}
& \tilde{v}^{\mu} D_{\mu}^{(a)} \bar{\Lambda}+i v^{\mu} D_{\mu}^{(a)} \bar{\Lambda}^{\prime}+\frac{1}{\ell}\left(w \bar{\Lambda}-\bar{\Lambda}^{\prime}\right)-\alpha\left(\eta_{0}\right)\left(\bar{\Lambda}+w \bar{\Lambda}^{\prime}\right)=\left(\nu-i \alpha\left(\sigma_{0}\right)\right)\left(w \bar{\Lambda}+\bar{\Lambda}^{\prime}\right), \\
& i v^{\mu} D_{\mu}^{(a)} \bar{\Lambda}+\tilde{v}^{\mu} D_{\mu}^{(a)} \bar{\Lambda}^{\prime}-\frac{1}{\ell}\left(\bar{\Lambda}-w \bar{\Lambda}^{\prime}\right)+\alpha\left(\eta_{0}\right)\left(w \bar{\Lambda}+\bar{\Lambda}^{\prime}\right)=-\left(\nu-i \alpha\left(\sigma_{0}\right)\right)\left(\bar{\Lambda}+w \bar{\Lambda}^{\prime}\right) .
\end{aligned}
$$

We can rewrite equations (3.87) and (3.88) into the following forms

$$
\begin{array}{r}
-\left(\nu-i \alpha\left(\sigma_{0}\right)\right)\left[w\left(w_{-} \Lambda+w_{+} \bar{\Lambda}\right)-\left(w_{-} \Lambda^{\prime}-w_{+} \bar{\Lambda}^{\prime}\right)\right]-i v^{\mu} D_{\mu}^{(a)}\left(w_{-} \Lambda^{\prime}-w_{+} \bar{\Lambda}^{\prime}\right)=0 \\
-i\left(\nu-i \alpha\left(\sigma_{0}\right)\right)\left[w_{-} \Lambda-w_{+} \bar{\Lambda}-w\left(w_{-} \Lambda^{\prime}+w_{+} \bar{\Lambda}^{\prime}\right)\right]-i \tilde{v}^{\mu} D_{\mu}^{(a)}\left(w_{-} \Lambda^{\prime}-w_{+} \bar{\Lambda}^{\prime}\right)=0 \\
\left(\nu-i \alpha\left(\sigma_{0}\right)\right)\left(w_{-} \Lambda+w_{+} \bar{\Lambda}\right)-\alpha\left(\eta_{0}\right)\left(w_{-} \Lambda^{\prime}-w_{+} \bar{\Lambda}^{\prime}\right)=0 .
\end{array}
$$

where $w_{ \pm}$is defined by (A.20). If we make the ansatz,

$$
\Lambda=f(\theta) e^{i(m+1) \varphi}, \quad \Lambda^{\prime}=g(\theta) e^{i(m+1) \varphi}, \quad \bar{\Lambda}=\bar{f}(\theta) e^{i(m-1) \varphi}, \quad \bar{\Lambda}^{\prime}=\bar{g}(\theta) e^{i(m-1) \varphi},
$$

where $m$ is an integer, then (3.95) and (3.97) can be written as

$$
\begin{array}{r}
-\ell\left(\nu-i \alpha\left(\sigma_{0}\right)\right) \cos \theta(f+\bar{f})+\left[\ell\left(\nu-i \alpha\left(\sigma_{0}\right)\right)+m-\ell \alpha\left(\eta_{0}\right)(\kappa-\cos \theta)\right](g-\bar{g})=0, \\
\ell\left(\nu-i \alpha\left(\sigma_{0}\right)\right)(f+\bar{f})-\ell \alpha\left(\eta_{0}\right)(g-\bar{g})=0 .
\end{array}
$$

These lead to

$$
\left[\ell\left(\nu-i \alpha\left(\sigma_{0}\right)\right)+m-\kappa \ell \alpha\left(\eta_{0}\right)\right](g-\bar{g})=0 .
$$

If $g-\bar{g} \neq 0,18$ we have

$$
\ell \nu=i \ell \alpha\left(\sigma_{0}\right)-m+\kappa \ell \alpha\left(\eta_{0}\right)
$$

\footnotetext{
18 We can see that, if $g-\bar{g}=0$, there are no consistent solutions with the regularity at $\theta=0$ and the boundary conditions at $\theta=\theta_{0}$, except the case that $\nu=i \alpha\left(\sigma_{0}\right), m=0$ and $\eta_{0}=0$.
} 
On the other hand, because (3.96) should be consistent with (3.91) and (3.94), we obtain a relation

$$
\left(-m+\kappa \ell \alpha\left(\eta_{0}\right)\right)(f-\bar{f})=\ell \alpha\left(\eta_{0}\right)(g+\bar{g}) .
$$

Thus, we obtain the following relations:

$$
\begin{gathered}
\left(-m+\kappa \ell \alpha\left(\eta_{0}\right)\right) f=\ell \alpha\left(\eta_{0}\right) g \\
\left(-m+\kappa \ell \alpha\left(\eta_{0}\right)\right) \bar{f}=-\ell \alpha\left(\eta_{0}\right) \bar{g} .
\end{gathered}
$$

From the remaining conditions, $g(\theta)$ should satisfy

$$
\sin \theta \partial_{\theta} g+\left[\left(m+1-\kappa \ell \alpha\left(\eta_{0}\right)\right) \cos \theta+\ell \alpha\left(\eta_{0}\right)\right] g=0
$$

and $\bar{g}(\theta)$ should satisfy

$$
\sin \theta \partial_{\theta} \bar{g}+\left[\left(-m+1+\kappa \ell \alpha\left(\eta_{0}\right)\right) \cos \theta-\ell \alpha\left(\eta_{0}\right)\right] \bar{g}=0
$$

One can show that general solutions of these differential equations are given by

$$
\begin{aligned}
& g(\theta)=C_{1}\left(-m+\kappa \ell \alpha\left(\eta_{0}\right)\right)\left(\sin \frac{\theta}{2}\right)^{(\kappa-1) \ell \alpha\left(\eta_{0}\right)}\left(\cos \frac{\theta}{2}\right)^{(\kappa+1) \ell \alpha\left(\eta_{0}\right)} \sin ^{-m-1} \theta, \\
& \bar{g}(\theta)=C_{2}\left(-m+\kappa \ell \alpha\left(\eta_{0}\right)\right)\left(\sin \frac{\theta}{2}\right)^{-(\kappa-1) \ell \alpha\left(\eta_{0}\right)}\left(\cos \frac{\theta}{2}\right)^{-(\kappa+1) \ell \alpha\left(\eta_{0}\right)} \sin ^{m-1} \theta .
\end{aligned}
$$

In the case where $\theta_{0}<\pi$, taking account of the regularity of fermionic eigenmodes at $\theta=0$ and the boundary condition (3.90), it is needed that $m=0$ and

$$
C_{1}\left(\cos \frac{\theta_{0}}{2}\right)^{2 \ell \alpha\left(\eta_{0}\right)}-C_{2}\left(\cos \frac{\theta_{0}}{2}\right)^{-2 \ell \alpha\left(\eta_{0}\right)}=0 .
$$

Therefore, the eigenvalue is given by

$$
\ell \nu=i \ell \alpha\left(\sigma_{0}\right)+\ell \alpha\left(\eta_{0}\right)
$$

For the special case that $\theta_{0}=\pi$, there are the following solutions:

$$
\begin{aligned}
& C_{2}=0, \\
& \ell \nu=i \ell \alpha\left(\sigma_{0}\right)-m+\ell \alpha\left(\eta_{0}\right), \quad \begin{cases}m \leq 0 & \text { for } \quad 2 \ell \alpha\left(\eta_{0}\right) \geq 2 \\
m \leq 2 \ell \alpha\left(\eta_{0}\right)-2 & \text { for } \quad 2 \ell \alpha\left(\eta_{0}\right) \leq 1\end{cases}
\end{aligned}
$$

or

$$
\begin{aligned}
& C_{1}=0, \\
& \ell \nu=i \ell \alpha\left(\sigma_{0}\right)-m+\ell \alpha\left(\eta_{0}\right), \quad \begin{cases}m \geq 0 & \text { for } 2 \ell \alpha\left(\eta_{0}\right) \leq-2 \\
m>2 \ell \alpha\left(\eta_{0}\right)+1 & \text { for } \quad 2 \ell \alpha\left(\eta_{0}\right) \geq-1\end{cases}
\end{aligned}
$$


In the case for $S^{2}$, there are solutions:

$$
\begin{aligned}
& C_{2}=0 \\
& \ell \nu=\left\{\begin{array}{lll}
i \ell \alpha\left(\sigma_{0}\right)-m+\left|\ell \alpha\left(\eta_{0}\right)\right| & \text { for } \ell \alpha\left(\eta_{0}\right) \neq 0 \\
i \ell \alpha\left(\sigma_{0}\right)-m+1 & \text { for } \ell \alpha\left(\eta_{0}\right)=0
\end{array} \quad(m \leq 0)\right.
\end{aligned}
$$

or

$$
\begin{aligned}
& C_{1}=0 \\
& \ell \nu=\left\{\begin{array}{ll}
i \ell \alpha\left(\sigma_{0}\right)-m-\left|\ell \alpha\left(\eta_{0}\right)\right| & \text { for } \ell \alpha\left(\eta_{0}\right) \neq 0 \\
i \ell \alpha\left(\sigma_{0}\right)-m-1 & \text { for } \ell \alpha\left(\eta_{0}\right)=0
\end{array} \quad(m \geq 0)\right.
\end{aligned}
$$

Therefore, up to an overall constant, the 1-loop factor for the vector multiplet is given by

$$
Z_{\mathrm{vector}}^{1-\mathrm{loop}}=\prod_{\alpha \in \Delta_{+}}\left(i \alpha\left(\sigma_{0}\right)+\alpha\left(\eta_{0}\right)\right),
$$

for $\theta_{0}<\pi$. For $\theta_{0}=\pi$, the 1-loop factor is

$$
\begin{aligned}
Z_{\text {vector }}^{1-\text { loo }}= & \prod_{\alpha \in \Delta_{+}} \prod_{\left\{\ell \alpha\left(\eta_{0}\right), m\right\} \in D_{f}}\left(i \ell \alpha\left(\sigma_{0}\right)-m+\ell \alpha\left(\eta_{0}\right)\right) \\
& \times \prod_{\alpha \in \Delta_{+}} \prod_{\left\{\ell \alpha\left(\eta_{0}\right), m\right\} \in D_{b}}\left(i \ell \alpha\left(\sigma_{0}\right)-m+\ell \alpha\left(\eta_{0}\right)\right)^{-1} \\
= & \prod_{\alpha \in \Delta_{+},\left|2 \ell \alpha\left(\eta_{0}\right)\right| \geq 2}\left(i \ell \alpha\left(\sigma_{0}\right)+\ell \alpha\left(\eta_{0}\right)\right),
\end{aligned}
$$

where

$$
\begin{aligned}
& D_{f}=\left\{\left\{2 \ell \alpha\left(\eta_{0}\right) \geq 2, m \leq 0\right\} \cup\left\{2 \ell \alpha\left(\eta_{0}\right) \leq 1, m \leq 2 \ell \alpha\left(\eta_{0}\right)-2\right\}\right. \\
& \left.\cup\left\{2 \ell \alpha\left(\eta_{0}\right) \leq-2, m \geq 0\right\} \cup\left\{2 \ell \alpha\left(\eta_{0}\right) \geq-1, m \geq 2 \ell \alpha\left(\eta_{0}\right)+2\right\}\right\},
\end{aligned}
$$

and

$$
\begin{gathered}
D_{b}=\left\{\left\{2 \ell \alpha\left(\eta_{0}\right) \geq 1, m \leq-1\right\} \cup\left\{2 \ell \alpha\left(\eta_{0}\right) \leq 0, m \leq 2 \ell \alpha\left(\eta_{0}\right)-2\right\}\right. \\
\left.\cup\left\{2 \ell \alpha\left(\eta_{0}\right) \leq-1, m \geq 1\right\} \cup\left\{2 \ell \alpha\left(\eta_{0}\right) \geq 0, m \geq 2 \ell \alpha\left(\eta_{0}\right)+2\right\}\right\} .
\end{gathered}
$$

For $S^{2}$, the 1-loop factor is given by

$$
\begin{aligned}
Z_{\text {vector }}^{1-\text { loop }}= & \prod_{\alpha \in \Delta_{+}, \alpha\left(\eta_{0}\right)=0} \prod_{m \geq 0}\left(\ell^{2} \alpha\left(\sigma_{0}\right)^{2}+(m+1)^{2}\right) \prod_{\alpha \in \Delta_{+}, \alpha\left(\eta_{0}\right) \neq 0} \prod_{m \geq 0}\left(\ell^{2} \alpha\left(\sigma_{0}\right)^{2}+\left(m+\left|\ell \alpha\left(\eta_{0}\right)\right|\right)^{2}\right) \\
& \times \prod_{\alpha \in \Delta_{+}} \prod_{m \geq 1}\left(\ell^{2} \alpha\left(\sigma_{0}\right)^{2}+\left(m+\left|\ell \alpha\left(\eta_{0}\right)\right|\right)^{2}\right)^{-1} \\
= & \prod_{\alpha \in \Delta_{+}, \alpha\left(\eta_{0}\right) \neq 0}\left(\ell^{2} \alpha\left(\sigma_{0}\right)^{2}+\ell^{2} \alpha\left(\eta_{0}\right)^{2}\right)
\end{aligned}
$$

The result for $S^{2}$ is same as [13, 14]. 


\subsubsection{Chiral multiplet}

Next let us consider the 1-loop determinant for the chiral multiplet.

Expanding fields around the saddle point and leaving only the quadratic terms, we have

$$
t \int \mathrm{d}^{2} x \sqrt{g} \delta V_{\text {chiral }}=\int \mathrm{d}^{2} x \sqrt{g} \mathcal{L}_{\text {reg }}+\mathcal{O}\left(t^{-1 / 2}\right),
$$

where

$$
\begin{aligned}
\mathcal{L}_{\mathrm{reg}} & =\bar{\phi} \Delta_{\phi} \phi+\bar{\psi} \Delta_{\psi} \psi, \\
\Delta_{\phi} & =-D_{\mu}^{(a)} D^{(a) \mu}+\sigma_{0}^{2}+\eta_{0}^{2}+i \frac{q-1}{\ell} \sigma_{0}-\frac{q(q-2)}{4 \ell^{2}}, \\
\Delta_{\psi} & =-i \gamma^{\mu} D_{\mu}^{(a)}+i \sigma_{0}-\eta_{0} \gamma^{3}-\frac{q}{2 \ell} .
\end{aligned}
$$

Hence, to evaluate the 1-loop determinant, we consider the eigenvalue problems for $\Delta_{\phi}$ and $\Delta_{\psi}$. Hereafter, we set $\beta=i \sigma_{0}-(q-1) / 2 \ell$.

We can see that there are partial cancellations in the 1-loop factor between the contributions from the bosonic and fermionic eigenmodes. Let $\psi$ be a fermionic eigenmode: $\Delta_{\psi} \psi=\nu \psi$. Then, if we define $\phi_{1} \equiv \bar{\epsilon} \psi$, we find that $\phi_{1}$ is a scalar eigenmode : $\Delta_{\phi} \phi_{1}=$ $\nu(\nu-2 \beta) \phi_{1}$. On the other hand, using a scalar eigenmode $\left(\Delta_{\phi} \phi=M^{2} \phi\right)$, we define

$$
\psi_{ \pm} \equiv\left(\nu_{ \pm}-\beta+\frac{1}{2 \ell}\right) \epsilon \phi-i \gamma^{\mu} \epsilon D_{\mu}^{(a)} \phi-\eta_{0} \gamma^{3} \epsilon \phi,
$$

where $\nu_{ \pm} \equiv \beta \pm \sqrt{M^{2}+\beta^{2}}$. Then, we find that

$$
\Delta_{\psi} \psi_{ \pm}=\nu_{ \pm} \psi_{ \pm}
$$

Note that $\phi_{1}$ and $\psi_{ \pm}$satisfy the boundary conditions (3.18).

\section{Unpaired eigenmodes}

We consider the unpaired fermionic eigenmode. If $\phi_{1}(\equiv \bar{\epsilon} \psi)=0, \psi$ can be written as $\psi=\bar{\epsilon} \Psi$, where $\Psi$ is a scalar function on which any boundary condition is not imposed. Since $\psi$ is a fermionic eigenmode, $\Delta_{\psi} \psi=\nu \psi$, we have

$$
\begin{aligned}
& i v^{\mu} D_{\mu}^{(a)} \Psi=\left(\nu-\beta-\frac{1}{2 \ell}-w \eta_{0}\right) \Psi, \\
& \tilde{v}^{\mu} D_{\mu}^{(a)} \Psi=\left[w\left(\nu-\beta-\frac{1}{2 \ell}\right)-\eta_{0}\right] \Psi .
\end{aligned}
$$


Therefore, we obtain the solutions:

$$
\Psi \propto\left(\sin \frac{\theta}{2}\right)^{(\kappa-1) \ell \eta_{0}}\left(\cos \frac{\theta}{2}\right)^{(\kappa+1) \ell \eta_{0}} \sin ^{m} \theta e^{-i m \varphi}
$$

where $m$ is an integer. The corresponding eigenvalues for weights $\rho$ are given by

$$
\nu=i \rho\left(\sigma_{0}\right)-\frac{q-2}{2 \ell}+\rho\left(\eta_{0}\right)+\frac{m}{\ell} \quad(m \geq 0),
$$

in the case where $\theta_{0}<\pi$, and

$$
\nu=i \rho\left(\sigma_{0}\right)-\frac{q-2}{2 \ell}+\rho\left(\eta_{0}\right)+\frac{m}{\ell}, \quad \begin{cases}m \geq 0 & \text { for } \ell \rho\left(\eta_{0}\right) \geq 0 \\ m \geq-2 \ell \rho\left(\eta_{0}\right) & \text { for } \ell \rho\left(\eta_{0}\right) \leq 0\end{cases}
$$

in the case where $\theta_{0}=\pi$, and

$$
\nu=i \rho\left(\sigma_{0}\right)-\frac{q-2}{2 \ell}+\left|\rho\left(\eta_{0}\right)\right|+\frac{m}{\ell} \quad(m \geq 0),
$$

for $S^{2}$.

On the other hand, the unpaired bosonic eigenmodes, $\Delta_{\phi} \phi=M^{2} \phi$, should satisfy

$$
\left(\nu-\beta+\frac{1}{2 \ell}\right) \epsilon \phi-i \gamma^{\mu} \epsilon D_{\mu}^{(a)} \phi-\eta_{0} \gamma^{3} \epsilon \phi=0 \quad\left(\nu(\nu-2 \beta)=M^{2}\right)
$$

which is equivalent to

$$
\begin{aligned}
i v^{\mu} D_{\mu}^{(a)} \phi & =\left(\nu-\beta+\frac{1}{2 \ell}-w \eta_{0}\right) \phi, \\
\tilde{v}^{\mu} D_{\mu}^{(a)} \phi & =-\left[w\left(\nu-\beta+\frac{1}{2 \ell}\right)-\eta_{0}\right] \phi .
\end{aligned}
$$

It can be easily checked that these equations lead to $\Delta_{\phi} \phi=M^{2} \phi$. In the case where $\theta_{0}<\pi$, there is no nontrivial solution which satisfies equation (3.135) and the boundary condition $\phi \mid=0$ simultaneously. In the case where $\theta_{0}=\pi$ and the case for $S^{2}$, we obtain the solutions

$$
\phi \propto\left(\sin \frac{\theta}{2}\right)^{-(\kappa-1) \ell \eta_{0}}\left(\cos \frac{\theta}{2}\right)^{-(\kappa+1) \ell \eta_{0}} \sin ^{m} \theta e^{i m \varphi} .
$$

The corresponding eigenvalues are given by

$$
\nu=i \rho\left(\sigma_{0}\right)-\frac{q}{2 \ell}+\rho\left(\eta_{0}\right)-\frac{m}{\ell}, \quad\left\{\begin{array}{ll}
m \geq 2 \ell \rho\left(\eta_{0}\right)+1 & \text { for } \quad \ell \rho\left(\eta_{0}\right) \geq 0 \\
m \geq 0 & \text { for } \quad \ell \rho\left(\eta_{0}\right)<0
\end{array},\right.
$$

for $\theta_{0}=\pi$, and

$$
\nu=i \rho\left(\sigma_{0}\right)-\frac{q}{2 \ell}-\left|\rho\left(\eta_{0}\right)\right|-\frac{m}{\ell} \quad(m \geq 0),
$$


for $S^{2}$.

Therefore, up to an overall constant, the 1-loop determinant for the chiral multiplet is given by

$$
Z_{\text {chiral }}^{1-\text { loo }}=\prod_{\rho} \prod_{m \geq 0}\left(i \ell \rho\left(\sigma_{0}\right)-\frac{q}{2}+\ell \rho\left(\eta_{0}\right)+m+1\right)
$$

for $\theta_{0}<\pi$. For the special case that $\theta_{0}=\pi$, we obtain 19

$$
\begin{aligned}
Z_{\text {chiral }}^{1-\text { loop }}= & \prod_{\rho \in \rho_{+}}\left(\frac{\prod_{m \geq 0}\left(i \ell \rho\left(\sigma_{0}\right)-\frac{q}{2}+\ell \rho\left(\eta_{0}\right)+m+1\right)}{\prod_{m \geq 2 \ell \rho\left(\eta_{0}\right)+1}\left(-i \ell \rho\left(\sigma_{0}\right)+\frac{q}{2}-\ell \rho\left(\eta_{0}\right)+m\right)}\right) \\
& \times \prod_{\rho \in \rho_{-}}\left(\frac{\prod_{m \geq-2 \ell \rho\left(\eta_{0}\right)}\left(i \ell \rho\left(\sigma_{0}\right)-\frac{q}{2}+\ell \rho\left(\eta_{0}\right)+m+1\right)}{\prod_{m \geq 0}\left(-i \ell \rho\left(\sigma_{0}\right)+\frac{q}{2}-\ell \rho\left(\eta_{0}\right)+m\right)}\right) .
\end{aligned}
$$

For $S^{2}$, the 1-loop factor is given by

$$
Z_{\text {chiral }}^{1-\text { loop }}=\prod_{\rho} \frac{\prod_{m \geq 0}\left(i \ell \rho\left(\sigma_{0}\right)-\frac{q}{2}+\left|\ell \rho\left(\eta_{0}\right)\right|+m+1\right)}{\prod_{m \geq 0}\left(-i \ell \rho\left(\sigma_{0}\right)+\frac{q}{2}+\left|\ell \rho\left(\eta_{0}\right)\right|+m\right)} .
$$

The result for $S^{2}$ is same as [13, 14].

\subsection{Partition functions and Wilson loops}

From what we have obtained, we find that the exact partition function for $\theta_{0}<\pi$ is given by

$$
Z=Z_{\text {classical }} Z_{\text {vector }}^{1-\text { loo }} Z_{\text {chiral }}^{1-\text { loop }}
$$

where

$$
Z_{\text {classical }}=e^{-i\left(\frac{\zeta}{\ell} \operatorname{Tr} \sigma_{0}+\frac{\Theta}{2 \pi \ell} \operatorname{Tr} \eta_{0}\right) V\left(\theta_{0}\right)}
$$

where $\sigma_{0}$ and $\eta_{0}$ were fixed at the boundary and $V\left(\theta_{0}\right)$ is the volume of the manifold we consider: $V\left(\theta_{0}\right)=2 \pi\left(1-\cos \theta_{0}\right) \ell^{2}$.

The supersymmetric Wilson loop operator is given by the following form

$$
W_{R}=\frac{1}{\operatorname{dim} \mathrm{R}} \operatorname{Tr}_{R} \mathrm{P} \exp \left(\oint_{\theta=\theta_{1}} \mathrm{~d} \varphi\left(i A_{\varphi}+\ell\left(-\sigma_{1}+i \cos \theta \sigma_{2}\right)\right)\right.
$$

\footnotetext{
${ }^{19} \rho_{+}\left(\right.$or $\left.\rho_{-}\right)$means the set of weight vectors such that $\rho\left(\eta_{0}\right) \geq 0\left(\right.$ or $\left.\rho\left(\eta_{0}\right)<0\right)$.
} 
where $R$ is a representation of the gauge group, and $\mathrm{P}$ represents path-ordering and its path is given by $\theta=\theta_{1},\left(0<\theta_{1}<\theta_{0}\right)$. This operator is actually invariant under the supersymmetry transformation generated by the Killing spinors (3.20). Thus, we find that the expectation value of the supersymmetric Wilson loop is exactly

$$
\left\langle W_{R}\right\rangle=\frac{1}{\operatorname{dim} \mathrm{R}} \operatorname{Tr}_{R} \exp \left(2 \pi\left(i \ell \eta_{0}-\ell \sigma_{0}\right)\right) .
$$

\section{Acknowledgments}

We would like to thank especially K. Hosomichi for helpful discussions on many points in the paper and K. Sakai for collaboration at the early stage of this work. We would also like to thank N. Hama and T. Nosaka for helpful discussions. S.T. was supported in part by JSPS KAKENHI Grant Number 23740189.

\section{A. Notations and useful formulas}

In this Appendix, we will explain the notations used in the paper and summarize some useful formulas.

Indices We use the following conventions for indices:

$$
\begin{array}{rc}
\text { coordinate indices } & \mu, \nu, \cdots \\
\text { tangent space indices } & a, b, \cdots \\
\text { spinor indices } & \alpha, \beta, \cdots
\end{array}
$$

Gamma matrices In this paper, we take gamma matrices as

$$
\gamma^{1}=\left(\begin{array}{ll}
0 & 1 \\
1 & 0
\end{array}\right), \gamma^{2}=\left(\begin{array}{cc}
0 & -i \\
i & 0
\end{array}\right), \gamma^{3}=\left(\begin{array}{cc}
1 & 0 \\
0 & -1
\end{array}\right)
$$

Spinors For both the three and two dimensional theories, we use the two-components Dirac spinors. The spinor-bi-linears are defined as

$$
\begin{aligned}
\bar{\epsilon} \lambda & \equiv \bar{\epsilon}_{\alpha} \lambda^{\alpha} \equiv C_{\alpha \beta} \bar{\epsilon}^{\alpha} \lambda^{\beta}, \\
\bar{\epsilon} \gamma^{a} \lambda & \equiv \bar{\epsilon}_{\alpha}\left(\gamma^{a}\right)_{\beta}^{\alpha} \lambda^{\beta},
\end{aligned}
$$


where $C_{\alpha \beta}$ is the antisymmetric matrix $\left(C_{12}=-C_{21}=1\right)$. It is easy to check that

$$
\bar{\epsilon} \lambda=\lambda \bar{\epsilon}, \quad \bar{\epsilon} \gamma^{a} \lambda=-\lambda \gamma^{a} \bar{\epsilon}
$$

for Grassmann odd spinors.

Useful formulas in 3D The Fierz identity for Grassmann odd spinors in 3D:

$$
(\eta \lambda)(\epsilon \psi)=-\frac{1}{2}(\eta \epsilon)(\lambda \psi)+\frac{1}{2}\left(\eta \gamma^{\mu} \epsilon\right)\left(\lambda \gamma_{\mu} \psi\right)
$$

Grassmann even positive Killing spinors on $S^{3}$ are spanned by

$$
\epsilon=\frac{1}{\sqrt{2}}\left(\begin{array}{c}
-e^{-\frac{i}{2}(\varphi-\chi-\theta)} \\
e^{-\frac{i}{2}(\varphi-\chi+\theta)}
\end{array}\right) \quad \text { and } \quad \bar{\epsilon}=\frac{1}{\sqrt{2}}\left(\begin{array}{c}
e^{\frac{i}{2}(\varphi-\chi+\theta)} \\
e^{\frac{i}{2}(\varphi-\chi-\theta)}
\end{array}\right) .
$$

The bi-linears of these Grassmann even spinors are given by

$$
\begin{aligned}
& \bar{\epsilon} \epsilon=1, \quad v^{a} \equiv \bar{\epsilon} \gamma^{a} \epsilon=(-\cos \theta, \sin \theta, 0), \\
& v_{+}^{a} \equiv \epsilon \gamma^{a} \epsilon=(i \sin \theta, i \cos \theta, 1) e^{-i(\varphi-\chi)}, \\
& v_{-}^{a} \equiv \bar{\epsilon} \gamma^{a} \bar{\epsilon}=(i \sin \theta, i \cos \theta,-1) e^{+i(\varphi-\chi)},
\end{aligned}
$$

We summarize some useful formulas for the above Killing spinors and Killing vectors:

$$
\begin{aligned}
v_{\mu} \gamma^{\mu} \epsilon & =\epsilon, \quad v_{\mu}^{+} \gamma^{\mu} \epsilon=0, \quad v_{\mu}^{-} \gamma^{\mu} \epsilon=2 \bar{\epsilon} \\
v_{\mu} \bar{\epsilon} \gamma^{\mu} & =\bar{\epsilon}, \quad v_{\mu}^{+} \bar{\epsilon} \gamma^{\mu}=2 \epsilon, \quad v_{\mu}^{-} \bar{\epsilon} \gamma^{\mu}=0, \\
v^{\mu} v_{\mu} & =1, \quad v_{+}^{\mu} v_{\mu}^{-}=-2, \quad \varepsilon_{a b c} v^{b} v_{ \pm}^{c}=\mp i v_{a}^{ \pm}, \quad \varepsilon_{a b c} v_{+}^{b} v_{-}^{c}=2 i v_{a}, \\
D_{\mu} v_{\nu}^{X} & =\frac{1}{\ell} \varepsilon_{\mu \nu \rho} v_{X}^{\rho} \quad(X=\text { no mark },+,-), \\
g^{\mu \nu} & =v^{\mu} v^{\nu}-\frac{1}{2}\left(v_{+}^{\mu} v_{-}^{\nu}+v_{-}^{\mu} v_{+}^{\nu}\right),
\end{aligned}
$$

and formulas for an arbitrary scalar function $Y$ :

$$
\begin{aligned}
{\left[v_{+}^{\mu} D_{\mu}^{(a)}, v_{-}^{\nu} D_{\nu}^{(a)}\right] Y } & =-\frac{4 i}{\ell} v^{\mu} D_{\mu}^{(a)} Y, \quad\left[v^{\mu} D_{\mu}^{(a)}, v_{ \pm}^{\nu} D_{\nu}^{(a)}\right] Y= \pm \frac{2 i}{\ell} v_{ \pm}^{\mu} D_{\mu}^{(a)} Y \\
D^{(a) \mu} D_{\mu}^{(a)} Y & =v^{\mu} D_{\mu}^{(a)}\left(v^{\nu} D_{\nu}^{(a)} Y\right)-\frac{1}{2} v_{+}^{\mu} D_{\mu}^{(a)}\left(v_{-}^{\nu} D_{\nu}^{(a)} Y\right)-\frac{1}{2} v_{-}^{\mu} D_{\mu}^{(a)}\left(v_{+}^{\nu} D_{\nu}^{(a)} Y\right) \\
& =v^{\mu} D_{\mu}^{(a)}\left(v^{\nu} D_{\nu}^{(a)} Y\right)-v_{-}^{\mu} D_{\mu}^{(a)}\left(v_{+}^{\nu} D_{\nu}^{(a)} Y\right)+\frac{2 i}{\ell} v^{\mu} D_{\mu}^{(a)} Y
\end{aligned}
$$

where $D_{\mu}^{(a)} Y \equiv\left(\nabla_{\mu}-i a_{\mu}\right) Y$ and $a_{\mu}$ is a background gauge field which satisfies $F_{\mu \nu}^{(a)} \equiv$ $\nabla_{\mu} a_{\nu}-\nabla_{\nu} a_{\mu}-i\left[a_{\mu}, a_{\nu}\right]=0$. 
Useful formulas in 2D The Fierz identity for Grassmann odd spinors in 2D is given by

$$
(\eta \lambda)(\epsilon \psi)=-\frac{1}{2}(\eta \epsilon)(\lambda \psi)+\frac{1}{2}\left(\eta \gamma^{3} \epsilon\right)\left(\lambda \gamma_{3} \psi\right)+\frac{1}{2}\left(\eta \gamma^{\mu} \epsilon\right)\left(\lambda \gamma_{\mu} \psi\right)
$$

Any Grassmann even positive Killing spinor on $S^{2}$ can be spanned by

$$
\epsilon=e^{i \frac{\varphi}{2}}\left(\begin{array}{c}
i \cos \frac{\theta}{2} \\
-\sin \frac{\theta}{2}
\end{array}\right) \quad \text { and } \quad \bar{\epsilon}=e^{-i \frac{\varphi}{2}}\left(\begin{array}{c}
-\sin \frac{\theta}{2} \\
i \cos \frac{\theta}{2}
\end{array}\right) .
$$

We can compute the bi-linears of these Grassmann even spinors:

$$
\begin{aligned}
\bar{\epsilon} \epsilon & =1, \quad w \equiv \bar{\epsilon} \gamma^{3} \epsilon=\cos \theta \\
v^{\mu} & \equiv \bar{\epsilon} \gamma^{\mu} \epsilon=(0,1 / \ell), \quad \tilde{v}^{\mu} \equiv \varepsilon^{\mu \nu} v_{\nu}=(\sin \theta / \ell, 0) \\
v_{+}^{\mu} & \equiv \epsilon \gamma^{\mu} \epsilon=-\frac{e^{i \varphi}}{\sin \theta}\left(\tilde{v}^{\mu}+i w v^{\mu}\right), \quad v_{-}^{\mu} \equiv \bar{\epsilon} \gamma^{\mu} \bar{\epsilon}=\frac{e^{-i \varphi}}{\sin \theta}\left(\tilde{v}^{\mu}-i w v^{\mu}\right), \\
w_{+} & \equiv \epsilon \gamma^{3} \epsilon=i e^{i \varphi} \sin \theta, \quad w_{-} \equiv \bar{\epsilon} \gamma^{3} \bar{\epsilon}=i e^{-i \varphi} \sin \theta
\end{aligned}
$$

We summarize some useful formulas for the above Killing spinors and their bi-linears:

$$
\begin{aligned}
v^{\mu} v_{\mu} & =\tilde{v}^{\mu} \tilde{v}_{\mu}=\sin ^{2} \theta=1-w^{2}, \quad v_{ \pm}^{\mu} v_{\mu}=-w w_{ \pm}, \quad v_{ \pm}^{\mu} \tilde{v}_{\mu}= \pm i w_{ \pm}, \\
D_{\mu} v_{\nu} & =\frac{1}{\ell} \varepsilon_{\mu \nu} w, \quad D_{\mu} \tilde{v}_{\nu}=\frac{1}{\ell} g_{\mu \nu} w, \quad D_{\mu} w=-\frac{1}{\ell} \varepsilon_{\mu \nu} v^{\nu}=-\frac{1}{\ell} \tilde{v}_{\mu}, \\
v^{\mu} \gamma_{\mu} \epsilon+w \gamma^{3} \epsilon & =\epsilon, \quad v^{\mu} \gamma_{\mu} \bar{\epsilon}+w \gamma^{3} \bar{\epsilon}=-\bar{\epsilon}, \quad \tilde{v}^{\mu} \gamma_{\mu} \epsilon=-i w_{+} \bar{\epsilon}, \tilde{v}^{\mu} \gamma_{\mu} \bar{\epsilon}=-i w_{-} \epsilon, \\
* D^{(a)} * D^{(a)} Y & =\frac{1}{1-w^{2}}\left[v^{\mu} D_{\mu}^{(a)}\left(v^{\nu} D_{\nu}^{(a)} Y\right)+\tilde{v}^{\mu} D_{\mu}^{(a)}\left(\tilde{v}^{\nu} D_{\nu}^{(a)} Y\right)\right],
\end{aligned}
$$

where $Y$ is an arbitrary scalar function and $D_{\mu}^{(a)} Y \equiv\left(\nabla_{\mu}-i a_{\mu}\right) Y$ and $a_{\mu}$ is a background gauge field.

\section{B. Supersymmetry variations}

In this Appendix, we confirm that the supersymmetry variations of actions can be written by surface terms which vanish by imposing the boundary conditions.

Three-dimensional theories We summarize the variation of the actions under the supersymmetry transformations (2.9)-(2.10), where we assume that SUSY parameters, $\epsilon, \bar{\epsilon}$, are positive Killing spinors. 
Yang-Mills Lagrangian:

$$
\begin{aligned}
\delta \mathcal{L}_{\mathrm{YM}}=\frac{1}{4} \operatorname{Tr} D_{\mu}[ & \frac{1}{2} \varepsilon^{\mu \nu \rho} F_{\nu \rho}(\bar{\lambda} \epsilon+\lambda \bar{\epsilon})+D_{\nu} \sigma\left(\bar{\lambda} \gamma^{\mu \nu} \epsilon-\lambda \gamma^{\mu \nu} \bar{\epsilon}\right) \\
& \left.+i F^{\mu \nu}\left(\bar{\lambda} \gamma_{\nu} \epsilon+\lambda \gamma_{\nu} \bar{\epsilon}\right)-D^{\mu} \sigma(\bar{\lambda} \epsilon-\lambda \bar{\epsilon})-i(D+\sigma / \ell)\left(\bar{\lambda} \gamma^{\mu} \epsilon-\lambda \gamma^{\mu} \bar{\epsilon}\right)\right] .
\end{aligned}
$$

Chern-Simons term:

$$
\delta \mathcal{L}_{\mathrm{CS}}=-\frac{i}{2} \operatorname{Tr} D_{\mu}\left[\varepsilon^{\mu \nu \rho} A_{\nu}\left(\bar{\lambda} \gamma_{\rho} \epsilon+\lambda \gamma_{\rho} \bar{\epsilon}\right)+2 \sigma\left(\bar{\lambda} \gamma^{\mu} \epsilon-\lambda \gamma^{\mu} \bar{\epsilon}\right)\right]
$$

FI term:

$$
\delta \mathcal{L}_{\mathrm{FI}}=\frac{\zeta}{2 \pi \ell} \operatorname{Tr} D_{\mu}\left(\bar{\lambda} \gamma^{\mu} \epsilon-\lambda \gamma^{\mu} \bar{\epsilon}\right)
$$

The matter kinetic terms:

$$
\begin{aligned}
\delta \mathcal{L}_{\mathrm{mat}}=\frac{1}{2} D_{\mu}\left(\bar{\psi} \epsilon D^{\mu} \phi-\bar{\psi} \gamma^{\mu \nu} \epsilon D_{\nu} \phi+i \bar{F} \epsilon \gamma^{\mu} \psi+\bar{\phi} \bar{\lambda} \gamma^{\mu} \epsilon \phi-\bar{\psi} \gamma^{\mu} \epsilon \sigma \phi-i \frac{q}{\ell} \bar{\psi} \gamma^{\mu} \epsilon \phi\right. \\
\left.+D^{\mu} \bar{\phi} \bar{\epsilon} \psi+D_{\nu} \bar{\phi} \bar{\epsilon} \gamma^{\mu \nu} \psi-i \bar{\psi} \gamma^{\mu} \bar{\epsilon} F-\bar{\phi} \lambda \gamma^{\mu} \bar{\epsilon} \phi+\bar{\phi} \sigma \bar{\epsilon} \gamma^{\mu} \psi+i \frac{q}{\ell} \bar{\phi} \bar{\epsilon} \gamma^{\mu} \psi\right)
\end{aligned}
$$

Therefore, the supersymmetry variations of actions can be written by surface terms, and actually they vanish if we assume that the Killing spinors satisfy the relations (2.19) and (2.21) and the fields satisfy the boundary conditions (2.16) $-(2.18)$ and (2.20)

Two-dimensional theories We summarize the variation of the actions under the supersymmetry transformations (3.9)-(3.10), where we assume that SUSY parameters, $\epsilon$ and $\bar{\epsilon}$, are positive Killing spinors.

Yang-Mills Lagrangian:

$$
\begin{aligned}
\delta \mathcal{L}_{\mathrm{YM}}=\frac{1}{4} \operatorname{Tr} D_{\mu}[ & \left(F_{12}-\sigma_{2} / \ell\right)\left(\bar{\lambda} \gamma^{\mu} \gamma^{3} \epsilon+\lambda \gamma^{\mu} \gamma^{3} \bar{\epsilon}\right)+i\left[\sigma_{1}, \sigma_{2}\right]\left(\bar{\lambda} \gamma^{\mu} \gamma^{3} \epsilon-\lambda \gamma^{\mu} \gamma^{3} \bar{\epsilon}\right) \\
& +D_{\nu} \sigma_{1}\left(\bar{\lambda} \gamma^{\mu \nu} \epsilon-\lambda \gamma^{\mu \nu} \bar{\epsilon}\right)-i D_{\nu} \sigma_{2}\left(\bar{\lambda} \gamma^{\mu \nu} \gamma^{3} \epsilon+\lambda \gamma^{\mu \nu} \gamma^{3} \bar{\epsilon}\right) \\
& \left.-D^{\mu} \sigma_{1}(\bar{\lambda} \epsilon-\lambda \bar{\epsilon})+i D^{\mu} \sigma_{2}\left(\bar{\lambda} \gamma^{3} \epsilon-\lambda \gamma^{3} \bar{\epsilon}\right)-i\left(D+\sigma_{1} / \ell\right)\left(\bar{\lambda} \gamma^{\mu} \epsilon-\lambda \gamma^{\mu} \bar{\epsilon}\right)\right]
\end{aligned}
$$

FI term:

$$
\delta \mathcal{L}_{\mathrm{FI}}=-\operatorname{Tr} D_{\mu}\left[\frac{\zeta}{2}\left(\bar{\lambda} \gamma^{\mu} \epsilon-\lambda \gamma^{\mu} \bar{\epsilon}\right)+\frac{\Theta}{4 \pi} \varepsilon^{\mu \nu}\left(\bar{\lambda} \gamma_{\nu} \epsilon+\lambda \gamma_{\nu} \bar{\epsilon}\right)\right]
$$


The matter kinetic terms:

$$
\begin{aligned}
\delta \mathcal{L}_{\text {mat }}=\frac{1}{2} D_{\mu}\left(\bar{\psi} \epsilon D^{\mu} \phi-\bar{\psi} \gamma^{\mu \nu} \epsilon D_{\nu} \phi+i \bar{F} \epsilon \gamma^{\mu} \psi\right. & \\
& +\bar{\phi} \bar{\lambda} \gamma^{\mu} \epsilon \phi-\bar{\psi} \gamma^{\mu} \epsilon \sigma_{1} \phi+i \bar{\psi} \gamma^{\mu} \gamma^{3} \epsilon \sigma_{2} \phi-i \frac{q}{2 \ell} \bar{\psi} \gamma^{\mu} \epsilon \phi \\
& +D^{\mu} \bar{\phi} \bar{\epsilon} \psi+D_{\nu} \bar{\phi} \bar{\epsilon} \gamma^{\mu \nu} \psi-i \bar{\psi} \gamma^{\mu} \bar{\epsilon} F \\
& \left.-\bar{\phi} \lambda \gamma^{\mu} \bar{\epsilon} \phi+\bar{\phi} \sigma_{1} \bar{\epsilon} \gamma^{\mu} \psi-i \bar{\phi} \sigma_{2} \bar{\epsilon} \gamma^{3} \gamma^{\mu} \psi+i \frac{q}{2 \ell} \bar{\phi} \bar{\epsilon} \gamma^{\mu} \psi\right)
\end{aligned}
$$

Therefore, the supersymmetry variations of actions can be written by surface terms, and actually they vanish if we assume that the Killing spinors satisfy the conditions (3.19) and the bulk fields satisfy the boundary conditions (3.14)-(3.18).

\section{Cancellations in the 1-loop factor}

In this Appendix, we will show how the cancellations in the 1-loop factor for the chiral multiplet in the 3D SUSY gauge theories occur more precisely. We can see that

$$
\begin{aligned}
0= & {\left[\Delta_{\phi}, h_{\phi}\right]=\left[\Delta_{\phi}, v_{ \pm}^{\mu} D_{\mu}\right] } \\
0= & {\left[\Delta_{\psi}, h_{\psi}\right]=\left[\Delta_{\psi}, v_{ \pm}^{\mu} D_{\mu}\right], } \\
& {\left[h_{\phi}, v_{ \pm}^{\mu} D_{\mu}\right]=\mp 2 v_{ \pm}^{\mu} D_{\mu} }
\end{aligned}
$$

where $h_{\phi} / \ell \equiv i v^{\mu} D_{\mu}$ and $h_{\psi} / \ell \equiv i v^{\mu} D_{\mu}-\frac{1}{2 \ell} v^{\mu} \gamma_{\mu}$. Thus, the eigenmodes $\Delta$ can be chosen as the eigenmodes of $h$. We can easily see that $h_{\psi} \epsilon=-\epsilon, h_{\psi} \bar{\epsilon}=\bar{\epsilon}$ and $h_{\phi} e^{i(m \varphi-n \chi)}=$ $(m+n) e^{i(m \varphi-n \chi)}$. Thus, for $\psi$ with $h_{\psi} \psi=h(\psi) \psi$, we have $h_{\phi} \phi_{1}=(h(\psi)+1) \phi_{1}$ where $\phi_{1}=\bar{\epsilon} \psi$. On the other hand, for $\phi$ with $h_{\phi} \phi=h(\phi) \phi$, we have $h_{\psi} \psi_{ \pm}=(h(\phi)-1) \psi_{ \pm}$where $\psi_{ \pm} \equiv\left(\nu_{ \pm}-\omega+\frac{1}{\ell}\right) \epsilon \phi-i \gamma^{\mu} \epsilon D_{\mu}^{(a)} \phi$ where $\nu_{ \pm} \equiv \omega \pm \sqrt{M^{2}+\omega^{2}}$ with $\Delta_{\phi} \phi=M^{2} \phi$.

If we construct $\psi_{+}$from a $\phi_{1}$ corresponding to an eigenmode $\psi$ with the eigenvalue $\nu$, we can show that

$$
\psi_{+}=(h(\psi) / \ell+w-\nu) \psi
$$

which is proportional to the original $\psi$ except $h(\psi) / \ell+w-\nu=0$. We can see that the eigenmode with $h(\psi) / \ell=-w+\nu$ are the "lowest" modes for $h$ with fixed $\Delta$.

Conversely, if we construct $\phi_{1}$ from a $\psi_{ \pm}$corresponding to an eigenmode $\phi$ with the eigenvalue $M^{2}=\nu(\nu-2 w)$, we can show that

$$
\phi_{1}=( \pm(\nu-w)+1 / \ell-h(\phi) / \ell) \phi,
$$


which is proportional to the original $\phi$ except $\pm(\nu-w)+1 / \ell-h(\phi) / \ell=0$. Therefore, the unpaired modes are the lowest and highest modes for $h$ of $\psi$ and $\phi$, respectively. (For $\bar{\phi}$ and $\bar{\psi}$, we can also show the same conclusion.) This conclusion is, of course, consistent with the discussions in section 2 .

\section{References}

[1] N. Seiberg, "Exact results on the space of vacua of four-dimensional SUSY gauge theories," Phys. Rev. D 49 (1994) 6857 hep-th/9402044; "Electric - magnetic duality in supersymmetric nonAbelian gauge theories," Nucl. Phys. B 435 (1995) 129 hep-th/9411149.

[2] N. Seiberg and E. Witten, "Electric - magnetic duality, monopole condensation, and confinement in N=2 supersymmetric Yang-Mills theory," Nucl. Phys. B 426 (1994) 19 [Erratum-ibid. B 430 (1994) 485] hep-th/9407087); "Monopoles, duality and chiral symmetry breaking in N=2 supersymmetric QCD," Nucl. Phys. B 431 (1994) 484 hep-th/9408099.

[3] K. A. Intriligator and N. Seiberg, "Lectures on supersymmetric gauge theories and electric - magnetic duality," Nucl. Phys. Proc. Suppl. 45BC (1996) 1 hep-th/9509066.

[4] N. A. Nekrasov, "Seiberg-Witten prepotential from instanton counting," Adv. Theor. Math. Phys. 7 (2004) 831 hep-th/0206161.

[5] V. Pestun, "Localization of gauge theory on a four-sphere and supersymmetric Wilson loops," arXiv:0712.2824 [hep-th].

[6] S. Kim, "The Complete superconformal index for N=6 Chern-Simons theory," Nucl. Phys. B 821 (2009) 241 [Erratum-ibid. B 864 (2012) 884] [arXiv:0903.4172 [hep-th]].

[7] A. Kapustin, B. Willett and I. Yaakov, "Exact Results for Wilson Loops in Superconformal Chern-Simons Theories with Matter," JHEP 1003 (2010) 089 arXiv:0909.4559 [hep-th]].

[8] D. L. Jafferis, "The Exact Superconformal R-Symmetry Extremizes Z," arXiv:1012.3210 [hep-th]. 
[9] N. Hama, K. Hosomichi and S. Lee, "Notes on SUSY Gauge Theories on Three-Sphere," JHEP 1103, 127 (2011) arXiv:1012.3512 [hep-th]].

[10] N. Hama, K. Hosomichi and S. Lee, "SUSY Gauge Theories on Squashed ThreeSpheres," JHEP 1105 (2011) 014 [arXiv:1102.4716 [hep-th]].

[11] Y. Imamura, "Relation between the 4d superconformal index and the $S^{3}$ partition function," JHEP 1109 (2011) 133 [arXiv:1104.4482 [hep-th]].

[12] Y. Imamura and D. Yokoyama, " $\mathrm{N}=2$ supersymmetric theories on squashed threesphere," Phys. Rev. D 85 (2012) 025015 [arXiv:1109.4734 [hep-th]].

[13] F. Benini and S. Cremonesi, "Partition functions of $N=(2,2)$ gauge theories on $S^{2}$ and vortices," arXiv:1206.2356 [hep-th].

[14] N. Doroud, J. Gomis, B. Le Floch and S. Lee, "Exact Results in D=2 Supersymmetric Gauge Theories," JHEP 1305 (2013) 093 [arXiv:1206.2606 [hep-th]].

[15] J. Gomis and S. Lee, "Exact Kahler Potential from Gauge Theory and Mirror Symmetry," JHEP 1304 (2013) 019 [arXiv:1210.6022 [hep-th]].

[16] K. Ohta and Y. Yoshida, "Non-Abelian Localization for Supersymmetric Yang-MillsChern-Simons Theories on Seifert Manifold," arXiv:1205.0046 [hep-th].

[17] H. -C. Kim, S. Kim, E. Koh, K. Lee and S. Lee, "On instantons as Kaluza-Klein modes of M5-branes," JHEP 1112 (2011) 031 arXiv:1110.2175 [hep-th]].

[18] K. Hosomichi, R. -K. Seong and S. Terashima, "Supersymmetric Gauge Theories on the Five-Sphere," arXiv:1203.0371 [hep-th].

[19] J. Kallen and M. Zabzine, "Twisted supersymmetric 5D Yang-Mills theory and contact geometry," arXiv:1202.1956 [hep-th].

[20] J. Kallen, J. Qiu and M. Zabzine, "The perturbative partition function of supersymmetric 5D Yang-Mills theory with matter on the five-sphere," arXiv:1206.6008 [hep-th].

[21] H. -C. Kim and S. Kim, "M5-branes from gauge theories on the 5-sphere," arXiv:1206.6339 [hep-th].

[22] T. Kawano and N. Matsumiya, "5D SYM on 3D Sphere and 2D YM," arXiv:1206.5966 [hep-th]. 
[23] J. Kallen, J. Qiu and M. Zabzine, "The perturbative partition function of supersymmetric 5D Yang-Mills theory with matter on the five-sphere," arXiv:1206.6008 [hep-th].

[24] N. Hama and K. Hosomichi, "Seiberg-Witten Theories on Ellipsoids," arXiv:1206.6359 [hep-th].

[25] H. -C. Kim, S. -S. Kim and K. Lee, "5-dim Superconformal Index with Enhanced En Global Symmetry," JHEP 1210 (2012) 142 [arXiv:1206.6781 [hep-th]].

[26] S. Terashima, "On Supersymmetric Gauge Theories on $S^{4} \mathrm{x} S^{1}$," arXiv:1207.2163 [hepth].

[27] Y. Tachikawa, "4d partition function on $S^{1} \times S^{3}$ and 2d Yang-Mills with nonzero area," PTEP 2013 (2013) 013B01 arXiv:1207.3497 [hep-th]].

[28] Y. Imamura, "Supersymmetric theories on squashed five-sphere," PTEP 2013 (2013) 013B04 [arXiv:1209.0561 [hep-th]].

[29] M. Fujitsuka, M. Honda and Y. Yoshida, "Maximal super Yang-Mills theories on curved background with off-shell supercharges," JHEP 1301 (2013) 162 arXiv:1209.4320 [hepth]].

[30] H. -C. Kim and K. Lee, "Supersymmetric M5 Brane Theories on R x CP2," JHEP 1307 (2013) 072 arXiv:1210.0853 [hep-th]].

[31] Y. Fukuda, T. Kawano and N. Matsumiya, "5D SYM and 2D q-Deformed YM," Nucl. Phys. B 869 (2013) 493 [arXiv:1210.2855 [hep-th]].

[32] Y. Imamura, "Perturbative partition function for squashed $S^{5}$," arXiv:1210.6308 [hepth].

[33] H. -C. Kim, J. Kim and S. Kim, "Instantons on the 5-sphere and M5-branes," arXiv:1211.0144 [hep-th].

[34] Y. Asano, G. Ishiki, T. Okada and S. Shimasaki, "Exact results for perturbative partition functions of theories with SU(2-4) symmetry," JHEP 1302 (2013) 148 arXiv:1211.0364 [hep-th]].

[35] H. Samtleben, E. Sezgin and D. Tsimpis, "Rigid 6D supersymmetry and localization," JHEP 1303 (2013) 137 arXiv:1212.4706 [hep-th]]. 
[36] C. Cordova and D. L. Jafferis, "Five-Dimensional Maximally Supersymmetric YangMills in Supergravity Backgrounds," arXiv:1305.2886 [hep-th].

[37] J. Qiu and M. Zabzine, "5D Super Yang-Mills on $Y^{p, q}$ Sasaki-Einstein manifolds," arXiv:1307.3149 [hep-th].

[38] L. F. Alday, D. Martelli, P. Richmond and J. Sparks, "Localization on ThreeManifolds," arXiv:1307.6848 [hep-th].

[39] H. -C. Kim, S. Kim, S. -S. Kim and K. Lee, "The general M5-brane superconformal index," arXiv:1307.7660 [hep-th].

[40] H. Jockers, V. Kumar, J. M. Lapan, D. R. Morrison and M. Romo, "Two-Sphere Partition Functions and Gromov-Witten Invariants," arXiv:1208.6244 [hep-th].

[41] K. Hori, A. Iqbal and C. Vafa, "D-branes and mirror symmetry," hep-th/0005247.

[42] K. Hori, "Linear models of supersymmetric D-branes," hep-th/0012179.

[43] M. Herbst, K. Hori and D. Page, "Phases Of N=2 Theories In 1+1 Dimensions With Boundary," arXiv:0803.2045 [hep-th].

[44] D. V. Vassilevich, "Quantum corrections to the mass of the supersymmetric vortex," Phys. Rev. D 68 (2003) 045005 hep-th/0304267].

[45] D. S. Berman and D. C. Thompson, "Membranes with a boundary," Nucl. Phys. B 820 (2009) 503 [arXiv:0904.0241 [hep-th]].

[46] D. S. Berman, M. J. Perry, E. Sezgin and D. C. Thompson, "Boundary Conditions for Interacting Membranes," JHEP 1004 (2010) 025 [arXiv:0912.3504 [hep-th]].

[47] T. Okazaki and S. Yamaguchi, "Supersymmetric Boundary Conditions in Three Dimensional N = 2 Theories," arXiv:1302.6593 [hep-th].

[48] D. Gaiotto and E. Witten, "Supersymmetric Boundary Conditions in N=4 Super YangMills Theory," J. Statist. Phys. 135 (2009) 789 [arXiv:0804.2902 [hep-th]]; "Janus Configurations, Chern-Simons Couplings, And The theta-Angle in N=4 Super Yang-Mills Theory," JHEP 1006 (2010) 097 arXiv:0804.2907 [hep-th]]; "S-Duality of Boundary Conditions In N=4 Super Yang-Mills Theory," Adv. Theor. Math. Phys. 13 (2009) arXiv:0807.3720 [hep-th]]. 
[49] S. Terashima, "On M5-branes in N=6 Membrane Action," JHEP 0808 (2008) 080 arXiv:0807.0197 [hep-th]].

[50] J. Gomis, D. Rodriguez-Gomez, M. Van Raamsdonk and H. Verlinde, "A Massive Study of M2-brane Proposals," JHEP 0809 (2008) 113 [arXiv:0807.1074 [hep-th]].

[51] D. Honda and T. Okuda, "Exact results for boundaries and domain walls in 2d supersymmetric theories," arXiv:1308.2217 [hep-th].

[52] K. Hori and M. Romo, "Exact Results In Two-Dimensional (2,2) Supersymmetric Gauge Theories With Boundary," arXiv:1308.2438 [hep-th].

[53] P. Goddard, J. Nuyts and D. I. Olive, "Gauge Theories and Magnetic Charge," Nucl. Phys. B 125 (1977) 1. 